JOURNAL OF

SYMPLECTIC GEOMETRY

Volume 12, Number 2, 313-363, 2014

\title{
ON THE ANTI-DIAGONAL FILTRATION FOR THE HEEGAARD FLOER CHAIN COMPLEX OF A BRANCHED DOUBLE-COVER
}

\author{
EAMONN TWEEDY
}

\begin{abstract}
Seidel and Smith introduced the graded fixed-point symplectic Khovanov cohomology group $K h_{\text {symp, inv }}(K)$ for a knot $K \subset S^{3}$, as well as a spectral sequence converging to the Heegaard Floer homology group $\widehat{H F}\left(\Sigma(K) \#\left(S^{2} \times S^{1}\right)\right)$ with $E^{1}$-page isomorphic to a factor of $K h_{\text {symp, inv }}(K)[\mathbf{2 2}]$. There the authors proved that $K h_{\text {symp, inv }}$ is a knot invariant. We show here that the higher pages of their spectral sequence are knot invariants also.
\end{abstract}

\section{Introduction}

Heegaard Floer homology was introduced by Ozsváth and Szabó in [13], and has proven to be a very useful tool in studying manifolds of dimensions three and four. We will be particularly interested in the invariant $\widehat{H F}$, which assigns to a 3-manifold $M$ an abelian group $\widehat{H F}(M)$. Given a knot $K \subset S^{3}$, the present paper will study $\widehat{H F}\left(\Sigma(K) \#\left(S^{2} \times S^{1}\right)\right)$, where $\Sigma(K)$ is the two-fold cover of the sphere $S^{3}$ branched along the knot $K$. The Heegaard Floer homology of branched double-covers was studied in [14], in which Ozsváth and Szabó constructed a spectral sequence from the reduced Khovanov homology group $\widetilde{K h}(-K ; \mathbb{Z} / 2 \mathbb{Z})$ to the group $\widehat{H F}(\Sigma(K) ; \mathbb{Z} / 2 \mathbb{Z})$, where $-K$ denotes the mirror of $K$.

Given a presentation of a knot $K \subset S^{3}$ as the braid closure of a braid $b$, Seidel and Smith introduced in [21] the symplectic Khovanov cohomology group $K h_{\text {symp }}(b)$, which is defined by taking the Lagrangian Floer cohomology of two Lagrangian submanifolds inside an affine variety. Clearly there may be different braids, which have isotopic braid closures. However, Seidel and Smith proved in [21] that $K h_{\text {symp }}$ is a knot invariant. In [16], Rezazadegan proved the existence of a spectral sequence from $K h(L)$ to $K h_{\text {symp }}(L)$ 
with $\mathbb{Z} / 2 \mathbb{Z}$ coefficients. Recent work-in-progress of Abouzaid and Smith [1] indicates that in fact $\operatorname{rk}_{\mathbb{Q}} K h(L)=\operatorname{rk}_{\mathbb{Q}} K h_{\mathrm{symp}}(L)$.

Furthermore, by studying the fixed-point sets of an involution on the variety, Seidel and Smith further define in $[\mathbf{2 2}]$ the fixed-point symplectic Khovanov cohomology group $K h_{\mathrm{symp} \text {, inv }}(b)$ for a braid $b$. Via the choice of a particular holomorphic volume form, one obtains gradings (in the sense of $[\mathbf{2 0}]$ ) on the totally-real submanifolds $\mathcal{T}$ and $\mathcal{T}^{\prime}$ used to define $K h_{\text {symp, inv }}(b)$; the gradings on these submanifolds induce an absolute $\mathbb{Z}$-valued Maslov grading $\widetilde{R}$ on the set $\mathcal{T} \cap \mathcal{T}^{\prime}$.

We will consider braids in $B_{2 n}$, the braid group on $2 n$ strands (where $n \in \mathbb{N}$ ), and obtain knot diagrams by taking plat closures. Although Seidel and Smith $[\mathbf{2 1}, \mathbf{2 2}]$ and Manolescu [9] considered braid closures instead, our convention will be chosen for computational reasons (note that Waldron illustrated in [25] that $K h_{\text {symp }}$ can be defined for bridge diagrams coming from such plat closures). We will recall the definition for the set $\mathcal{G}$ of Bigelow generators, unordered $n$-tuples of distinct intersection points in a fork diagram obtained from the braid $b$. Following $[\mathbf{3}, \mathbf{9}]$, we will then define functions $Q, P, T: \mathcal{G} \rightarrow \mathbb{Z}$, which can be computed from this diagram in an elementary fashion.

In [9], Manolescu used the fork diagram to give a description of the group $K h_{\text {symp, inv }}(b)$, and in particular showed a one-to-one correspondence between $\mathcal{G}$ and a set of generators for the Seidel-Smith cochain complex. In this context, one can view the totally real submanifolds $\mathcal{T}$ and $\mathcal{T}^{\prime}$ as admissible Heegaard tori for the manifold $\Sigma(K) \#\left(S^{2} \times S^{1}\right)$. Thus the set $\mathcal{G}$ is also in one-to-one correspondence with a set of generators for the chain group $\widehat{C F}\left(\Sigma(K) \#\left(S^{2} \times S^{1}\right)\right)$. This identification provides a function $\widetilde{R}: \mathcal{G} \rightarrow \mathbb{Z}$, and following $[\mathbf{9}]$ we have that $\widetilde{R}=T-Q+P$.

The function $R$ is obtained from $\widetilde{R}$ by a rational shift $s_{R}$ which depends on some properties of the braid $b \in B_{2 n}$ and the knot diagram $D$ which is its plat closure. Let $e(b)$ be the signed count of braid generators in the word $b$ and let $w(D)$ be the writhe of the diagram $D$ for $K$ given by the plat closure of $b$. Then define

$$
R=\widetilde{R}+s_{R}(b, D), \quad \text { where } \quad s_{R}(b, D)=\frac{e(b)-w(D)-2 n}{4} .
$$

Furthermore, for $\mathfrak{s} \in \operatorname{Spin}^{c}(M)$ torsion, Ozsváth and Szabó used surgery cobordisms to define an absolute $\mathbb{Q}$-valued grading $\widetilde{g r}$ on the subcomplex $\widehat{C F}(M, \mathfrak{s})$ which is an absolute lift of the relative Maslov $\mathbb{Z}$-grading. Then for torsion $\mathfrak{s}$, we define a filtration $\rho$ on the Heegaard Floer chain complex $\widehat{C F}\left(\Sigma(K) \#\left(S^{2} \times S^{1}\right), \mathfrak{s}\right)$ by $\rho=R-\widetilde{g r}$.

Two braids with isotopic plat closures can be connected via a finite sequence of Birman moves [4], which in turn induce sequences of isotopies, handleslides, and stabilizations (and associated chain homotopy equivalences 
on the Heegaard Floer complexes). We will prove the following theorem about the filtration $\rho$ in Section 5.2:

Theorem 1.0.1. Let the braids $b \in B_{2 n}$ and $b^{\prime} \in B_{2 m}$ have plat closures, which are diagrams for the knot $K$. Let $\mathcal{H}$ and $\mathcal{H}^{\prime}$ be the pointed Heegaard diagrams for $\Sigma(K) \#\left(S^{2} \times S^{1}\right)$ induced by $b$ and $b^{\prime}$, respectively, in the sense of Proposition 4.2.1 below. Let $\mathfrak{s} \in \operatorname{Spin}^{c}\left(\Sigma(K) \#\left(S^{2} \times S^{1}\right)\right)$ be torsion. Then the $\rho$-filtered chain complexes $\widehat{C F}(\mathcal{H}, \mathfrak{s})$ and $\widehat{C F}\left(\mathcal{H}^{\prime}, \mathfrak{s}\right)$ have the same filtered chain homotopy type.

More concisely, we can state the following:

Corollary 1.0.2. For each torsion $\mathfrak{s} \in \operatorname{Spin}^{c}\left(\Sigma(K) \#\left(S^{2} \times S^{1}\right)\right)$, the $\rho$ filtered chain homotopy type of the complex $\widehat{C F}\left(\Sigma(K) \#\left(S^{2} \times S^{1}\right), \mathfrak{s}\right)$ is an invariant of $K$.

In a standard way, the filtration $\rho$ provides a spectral sequence (whose pages we will denote by $\left.E^{k}\right)$ computing the group $\widehat{H F}\left(\Sigma(K) \#\left(S^{2} \times S^{1}\right)\right)$. Furthermore, the page $E^{1}$ is isomorphic to the subgroup of $K h_{\text {symp, inv }}(b)$ obtained by taking cohomology of the subcomplex whose generators correspond to generators of $\widehat{C F}$ in the torsion $\operatorname{Spin}^{c}$ structures on $\Sigma(K) \#\left(S^{2} \times\right.$ $\left.S^{1}\right)$. This spectral sequence is the same as the one defined by Seidel and

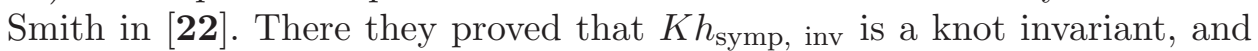
so the the factor corresponding to $E^{1}$ is also. Because higher pages are determined by the filtered chain homotopy type of $E^{0}$, Corollary 1.0 .2 implies the following.

Corollary 1.0.3. For $k \geq 1$, the page $E^{k}$ is a knot invariant.

Under certain degeneracy conditions of the spectral sequence, the function $R$ in fact provides a homological grading on Heegaard Floer theory. We say that a knot $K$ is $\rho$-degenerate if the spectral sequence collapses at $E^{1}$ and the induced filtration $\rho$ on $E^{\infty}$ is constant on each nontrivial factor $\widehat{H F}\left(\Sigma(K) \#\left(S^{2} \times S^{1}\right), \mathfrak{s}\right)$. The following is an easy consequence of the definitions.

Proposition 1.0.4. Let $K \subset S^{3}$ be a knot. Then the following are equivalent:

(i) $K$ is $\rho$-degenerate.

(ii) The filtration $R$ is a grading and lifts the relative Maslov $\mathbb{Z}$-grading on each nontrivial factor $\widehat{H F}\left(\Sigma(K) \#\left(S^{2} \times S^{1}\right), \mathfrak{s}\right)$.

Moreover, the grading $R$ is a knot invariant when the above hold.

\section{Topological preliminaries}

In [13], Ozsváth and Szabó define the Heegaard Floer homology group $\widehat{H F}(M)$ associated to a connected, closed, oriented 3-manifold $M$. A genus-g 
Heegaard splitting for such a manifold can be described via a pointed Heegaard diagram $\mathcal{H}=(\Sigma ; \boldsymbol{\alpha} ; \boldsymbol{\beta} ; z)$, where $\Sigma$ is the splitting surface, $\boldsymbol{\alpha}$ and $\boldsymbol{\beta}$ are g-tuples of attaching curves for the handlebodies, and $z \in\left(\Sigma-\cup \alpha_{i}-\cup \beta_{i}\right)$.

Definition 2.0.5. Let $(\Sigma ; \boldsymbol{\alpha} ; \boldsymbol{\beta} ; z)$ be a pointed Heegaard diagram, and let $D_{1}, \ldots, D_{m}$ be the connected components of $\Sigma \backslash\left(\cup \alpha_{i}\right) \backslash\left(\cup \beta_{i}\right)$, where $z \in D_{m}$. Then a two-chain

$$
\mathcal{P}:=\sum_{i=1}^{m-1} n_{i} D_{i} \quad \text { with } \quad n_{i} \in \mathbb{Z}
$$

is called a periodic domain if its boundary is a sum of $\alpha$ and $\beta$ circles.

Definition 2.0.6. A Heegaard diagram $(\Sigma ; \boldsymbol{\alpha} ; \boldsymbol{\beta} ; z)$ is called admissible if every periodic domain has both positive and negative coefficients.

If $\mathcal{H}$ is an admissible pointed Heegaard diagram, then one can compute the chain complex $\widehat{C F}(\mathcal{H})$ and its homology group $\widehat{H F}(M)$ is the Lagrangian Floer homology of the tori $\mathbb{T}_{\boldsymbol{\alpha}}$ and $\mathbb{T}_{\boldsymbol{\beta}}$ lying inside of the symplectic manifold $\operatorname{Sym}^{g}(\Sigma \backslash z)$.

More precisely, the group $\widehat{C F}(\mathcal{H})$ is generated by the set of intersections $\mathbb{T}_{\boldsymbol{\alpha}} \cap \mathbb{T}_{\boldsymbol{\beta}} \subset \operatorname{Sym}^{g}(\Sigma)$, and the differential is given by

$$
\widehat{\partial}(\mathbf{x})=\sum_{\mathbf{y} \in \mathbb{T}_{\boldsymbol{\alpha}} \cap \mathbb{T}_{\boldsymbol{\beta}}}\left(\sum_{\left\{\phi \in \pi_{2}(\mathbf{x}, \mathbf{y}) \mid \mu(\phi)=1, n_{z}(\phi)=0\right\}}(\# \widehat{\mathcal{M}}(\phi))\right) \mathbf{y},
$$

where $\widehat{\mathcal{M}}(\phi)$ denotes the reduced moduli space of pseudo-holomorphic representatives for the class $\phi, \mu(\phi)$ denotes the Maslov index of $\phi$, and $n_{z}(\phi):=\operatorname{Im}(\phi) \cap\left(\{z\} \cap \operatorname{Sym}^{g-1}(\Sigma)\right)$.

Recall that there is a function

$$
\mathfrak{s}_{z}: \mathbb{T}_{\boldsymbol{\alpha}} \cap \mathbb{T}_{\boldsymbol{\beta}} \longrightarrow \operatorname{Spin}^{c}(M)
$$

partitioning $\mathbb{T}_{\boldsymbol{\alpha}} \cap \mathbb{T}_{\boldsymbol{\beta}}$ into equivalence classes $\mathfrak{U}_{\mathfrak{s}}$. In fact, this function $\mathfrak{s}_{z}$ induces decompositions

$$
\widehat{C F}(\mathcal{H})=\bigoplus_{\mathfrak{s} \in \operatorname{Spin}^{c}(M)} \widehat{C F}(\mathcal{H}, \mathfrak{s}) \text { and } \widehat{H F}(M)=\bigoplus_{\mathfrak{s} \in \operatorname{Spin}^{c}(M)} \widehat{H F}(M, \mathfrak{s}) .
$$

For each $\mathfrak{s} \in \operatorname{Spin}^{c}(M)$ the chain complex $\widehat{C F}(M, \mathfrak{s})$ carries a relative grading $g r$ defined via the Maslov index. For $\mathfrak{s} \in \operatorname{Spin}^{c}(M)$ torsion, Ozsváth and Szabó use surgery cobordisms to construct in $[\mathbf{1 5}]$ an absolute $\mathbb{Q}$-valued grading $\widetilde{g r}$ on $\mathfrak{U}_{\mathfrak{s}}$ which lifts the relative grading in the following sense: if $\mathbf{x}, \mathbf{y} \in \mathfrak{U}_{\mathfrak{s}}$, then

$$
\widetilde{g r}(\mathbf{x})-\widetilde{g r}(\mathbf{y})=\operatorname{gr}(\mathbf{x}, \mathbf{y}) \text {. }
$$

Whenever $b_{1}(M)=0$, all $\operatorname{Spin}^{c}$ structures on $M$ are torsion and so the group $\widehat{H F}(M)$ can be absolutely graded via $\widetilde{g r}$. In particular, this holds for $M=\Sigma(K)$ for a knot $K \subset S^{3}$. However, although $\operatorname{Spin}^{c}\left(\Sigma(K) \#\left(S^{2} \times\right.\right.$ 
$\left.S^{1}\right)$ ) contains non-torsion elements, the group $\widehat{H F}\left(\Sigma(K) \#\left(S^{2} \times S^{1}\right), \mathfrak{s}\right)$ is nontrivial only if $\mathfrak{s}$ is torsion.

\subsection{3-gon chain maps and 4-gon homotopies.}

Remark 2.1.1. There can be some ambiguity surrounding terms like "triangle" and "quadrilateral", in particular when distinguishing between the polygons in the symmetric product $\operatorname{Sym}^{g}(\Sigma)$ and the regions which are their shadows in the surface $\Sigma$. We will follow Sarkar's convention in [18] in using neither of these words. The Whitney polygons in symmetric products will be referred to as $n$-gons and regions in surfaces will be referred to as $n$-sided regions.

In $[\mathbf{1 3}, \mathbf{1 5}]$, maps between Floer homologies are constructed by counting pseudo-holomorphic 3-gons in a certain equivalence class. We review these ideas below.

First recall the notion of a pointed Heegaard triple-diagram $(\Sigma ; \boldsymbol{\alpha} ; \boldsymbol{\beta} ; \boldsymbol{\gamma} ; z)$, where $\Sigma$ is an oriented two-manifold of genus $g, \boldsymbol{\alpha}, \boldsymbol{\beta}$, and $\boldsymbol{\gamma}$ are complete $g$-tuples of attaching circles for handlebodies $U_{\alpha}, U_{\beta}$, and $U_{\gamma}$, respectively, and $z \in\left(\Sigma \backslash \cup \alpha_{i} \backslash \cup \beta_{i} \backslash \cup \gamma_{i}\right)$. We then have pointed Heegaard diagrams $\mathcal{H}_{\boldsymbol{\alpha} \boldsymbol{\beta}}=(\Sigma ; \boldsymbol{\alpha} ; \boldsymbol{\beta} ; z), \mathcal{H}_{\boldsymbol{\beta} \boldsymbol{\gamma}}=(\Sigma ; \boldsymbol{\beta} ; \boldsymbol{\gamma} ; z)$, and $\mathcal{H}_{\boldsymbol{\alpha} \boldsymbol{\gamma}}=(\Sigma ; \boldsymbol{\alpha} ; \boldsymbol{\gamma} ; z)$, depicting manifolds $Y_{\boldsymbol{\alpha} \boldsymbol{\beta}}, Y_{\boldsymbol{\beta} \boldsymbol{\gamma}}$, and $Y_{\boldsymbol{\alpha} \boldsymbol{\gamma}}$, respectively. There is an analogous notion of a pointed Heegaard quadruple-diagram $(\Sigma ; \boldsymbol{\alpha} ; \boldsymbol{\beta} ; \boldsymbol{\gamma} ; \boldsymbol{\delta} ; z)$.

There are notions of triply-periodic domains in triple-diagrams and quadruply-periodic domains in quadruple-diagrams, and the definitions are analogous to that of a periodic domain. Multi-diagrams also have analogous notions of admissibility.

Definition 2.1.2. A pointed Heegaard triple-diagram (resp. quadruplediagram) is admissible if every triply-periodic domain (resp. quadruplyperiodic domain) has both positive and negative coefficients.

If the pointed triple-diagram $(\Sigma ; \boldsymbol{\alpha} ; \boldsymbol{\beta} ; \boldsymbol{\gamma} ; z)$ is admissible, then there is a chain map

given by the formula

$$
\widehat{f}_{\alpha \beta \gamma}: \widehat{C F}\left(\mathcal{H}_{\boldsymbol{\alpha} \boldsymbol{\beta}}\right) \otimes \widehat{C F}\left(\mathcal{H}_{\boldsymbol{\beta} \gamma}\right) \rightarrow \widehat{C F}\left(\mathcal{H}_{\boldsymbol{\alpha} \gamma}\right)
$$

$$
\widehat{f}_{\alpha \beta \gamma}(\mathbf{x} \otimes \mathbf{y})=\sum_{\mathbf{w} \in \mathbb{T}_{\boldsymbol{\alpha}} \cap \mathbb{T}_{\boldsymbol{\gamma}}}\left(\sum_{\left\{\psi \in \pi_{2}(\mathbf{x}, \mathbf{y}, \mathbf{w}) \mid \mu(\psi)=0, n_{z}(\psi)=0\right\}}(\# \mathcal{M}(\psi))\right) \mathbf{w}
$$

where $\mathcal{M}(\psi)$ is the moduli space of pseudo-holomorphic representatives for the class $\psi$. The induced map on homology will be denoted by $\widehat{F}_{\alpha \beta \gamma}$.

If the pointed quadruple-diagram $(\Sigma ; \boldsymbol{\alpha} ; \boldsymbol{\beta} ; \boldsymbol{\gamma} ; \boldsymbol{\delta} ; z)$ is admissible, then one can define a map

$$
\widehat{h}_{\alpha \beta \gamma \delta}: \widehat{C F}\left(\mathcal{H}_{\boldsymbol{\alpha} \boldsymbol{\beta}}\right) \otimes \widehat{C F}\left(\mathcal{H}_{\boldsymbol{\beta} \boldsymbol{\gamma}}\right) \otimes \widehat{C F}\left(\mathcal{H}_{\boldsymbol{\gamma} \boldsymbol{\delta}}\right) \rightarrow \widehat{C F}\left(\mathcal{H}_{\boldsymbol{\alpha} \boldsymbol{\delta}}\right)
$$


by the formula

$$
\widehat{h}_{\alpha \beta \gamma \delta}(\mathbf{x} \otimes \mathbf{y} \otimes \mathbf{w})=\sum_{\mathbf{z} \in \mathbb{T}_{\boldsymbol{\alpha}} \cap \mathbb{T}_{\delta}}\left(\sum_{\left\{\psi \in \pi_{2}(\mathbf{x}, \mathbf{y}, \mathbf{w}, \mathbf{z}) \mid \mu(\psi)=-1, n_{z}(\psi)=0\right\}}(\# \mathcal{M}(\psi))\right) \mathbf{z}
$$

A 4-gon map actually provides a chain homotopy between two compositions of 3-gon maps:

Theorem 2.1.3 ([13]). Let $(\Sigma ; \boldsymbol{\alpha} ; \boldsymbol{\beta} ; \boldsymbol{\gamma} ; \boldsymbol{\delta} ; z)$ be an admissible pointed Heegaard quadruple-diagram. Then for $\xi \in \widehat{C F}\left(\mathcal{H}_{\boldsymbol{\alpha} \boldsymbol{\beta}}\right), \eta \in \widehat{C F}\left(\mathcal{H}_{\boldsymbol{\beta} \gamma}\right)$, and $\zeta \in \widehat{C F}\left(\mathcal{H}_{\gamma \delta}\right)$,

$$
\begin{aligned}
& \partial \widehat{h}_{\alpha \beta \gamma \delta}(\xi \otimes \eta \otimes \zeta)+\widehat{h}_{\alpha \beta \gamma \delta}(\partial(\xi \otimes \eta \otimes \zeta)) \\
& \quad=\widehat{f}_{\alpha \gamma \delta}\left(\widehat{f}_{\alpha \beta \gamma}(\xi \otimes \eta) \otimes \zeta\right)-\widehat{f}_{\alpha \beta \delta}\left(\xi \otimes \widehat{f}_{\beta \gamma \delta}(\eta \otimes \zeta)\right)
\end{aligned}
$$

Classes of Whitney $n$-gons can be studied by examining their 'shadows' in the Heegaard surface $\Sigma$. We recall the definition of the domain of a 2-gon class, though there are analogous notions of domains of $n$-gon classes.

Definition 2.1.4. Let $(\Sigma ; \boldsymbol{\alpha} ; \boldsymbol{\beta} ; z)$ be a pointed Heegaard diagram, and denote by $\mathcal{D}_{0}, \mathcal{D}_{1}, \ldots, \mathcal{D}_{N}$ the connected components of $\Sigma \backslash\left(\cup_{i} \alpha_{i}\right) \backslash\left(\cup_{i} \beta_{i}\right)$, where $\mathcal{D}_{0}$ is the component containing the basepoint $z$. Then for $0 \leq j \leq N$, choose a point $z_{j}$ in the interior of $\mathcal{D}_{j}$. For some class $\phi \in \pi_{2}(\mathbf{x}, \mathbf{y})$ for $\mathbf{x}, \mathbf{y} \in \mathbb{T}_{\boldsymbol{\alpha}} \cap \mathbb{T}_{\boldsymbol{\beta}}$, the domain of $\phi$ is the 2-chain

$$
\mathcal{D}(\phi):=\sum_{j=0}^{N} n_{j} \mathcal{D}_{j} \quad \text { where } \quad n_{j}:=\operatorname{Im}(\phi) \cap\left(\left\{z_{j}\right\} \times \operatorname{Sym}^{g-1}(\Sigma)\right) .
$$

We will say that $\phi$ avoids the basepoint if $n_{0}=0$ (equivalently, $n_{z}(\phi)=0$ ).

2.1.1. Some index-zero 3 -gon classes. We are interested in 3-gon classes of Maslov index zero. To calculate index, we will follow Sarkar's work in [18] on Whitney $n$-gons, which we will review here. Some labeling conventions have been modified to fit our notation, and we will specialize to the $n=3$ case for this discussion.

Let $(\Sigma ; \boldsymbol{\alpha} ; \boldsymbol{\beta} ; \boldsymbol{\gamma} ; z)$ be an admissible pointed Heegaard triple-diagram, and let $\psi$ be a 3 -gon class connecting $\mathbf{x}, \mathbf{y}$, and $\mathbf{w}$ as defined above. Denote by $a(\psi), b(\psi)$, and $c(\psi)$ the boundaries $\left.\partial \mathcal{D}(\psi)\right|_{\alpha},\left.\partial \mathcal{D}(\psi)\right|_{\beta}$, and $\left.\partial \mathcal{D}(\psi)\right|_{\gamma}$, respectively.

Now given some 1-chains $a$ supported on $\alpha$ and $b$ supported on $\beta$, Sarkar defines the number b.a as follows. Assuming some orientation on the $\alpha$ and $\beta$ circles and on $\Sigma$, we have four well-defined directions in which we can translate $b$ so that no endpoint of $a$ lies on the translate $b^{\prime}$ and no endpoint of $b^{\prime}$ lies on $a$. These can be thought of as "northeast", "northwest", "southeast", and "southwest". After a small translation in some direction, we can 


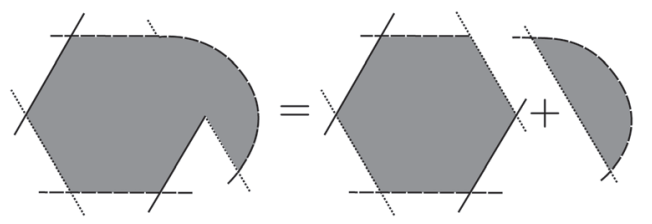

Figure 1. Decomposing the obtuse 6-sided component of $\mathcal{D}(\psi)$.

calculate the intersection number of $b^{\prime}$ with $a$. Then $b . a$ is defined to be the average of these numbers over the four possible translation directions.

Some element $\mathbf{x} \in \mathbb{T}_{\boldsymbol{\alpha}} \cap \mathbb{T}_{\boldsymbol{\beta}}$ is an unordered $g$-tuple $\left\{x_{1}, x_{2}, \ldots, x_{g}\right\}$. Define the number $\mu_{x}(\psi)=\sum \mu_{x_{i}}(\psi)$, where $\mu_{x_{i}}(\psi)$ is the average of the local coefficients of the 2-chain $\mathcal{D}(\psi)$ over the four quadrants around $x_{i} \in \Sigma$.

The Euler measure of $\mathcal{D}(\psi)$ will be denoted by $e(\psi)$. The Euler measure is additive, and it is enough for our purposes to know that the measure of an $n$-sided region is $(1-n / 4)$.

Equipped with these concepts, we present the following formula of Sarkar:

Theorem 2.1.5 ([18]). Let $(\Sigma ; \boldsymbol{\alpha} ; \boldsymbol{\beta} ; \boldsymbol{\gamma} ; z)$ be a pointed Heegaard triplediagram, and let $\psi \in \pi_{2}(\mathbf{x}, \mathbf{y}, \mathbf{w})$ be a 3-gon class connecting $\mathbf{x} \in \mathbb{T}_{\boldsymbol{\alpha}} \cap \mathbb{T}_{\boldsymbol{\beta}}$, $\mathbf{y} \in \mathbb{T}_{\boldsymbol{\beta}} \cap \mathbb{T}_{\boldsymbol{\gamma}}$, and $\mathbf{w} \in \mathbb{T}_{\boldsymbol{\alpha}} \cap \mathbb{T}_{\boldsymbol{\gamma}}$. Then the Maslov index $\mu(\psi)$ satisfies the formula

$$
\mu(\psi)=e(\psi)+\mu_{\mathbf{x}}(\psi)+\mu_{\mathbf{y}}(\psi)+a(\psi) \cdot c(\psi)-g / 2 .
$$

Here we will discuss two types of 3-gon classes in $\operatorname{Sym}^{g}(\Sigma)$.

A 3-gon $\psi$ of the first type has domain $\mathcal{D}(\psi)$ given by the sum of $g$ disjoint 3 -sided regions, each with coefficient +1 . A 3-gon $\psi$ of the second type has domain $\mathcal{D}\left(\psi^{\prime}\right)$ given by the sum of $(g-1)$ disjoint regions, consisting of $(g-2) 3$-sided regions and a single 6 -sided region with one angle larger than $\pi$, each with coefficient +1 . Components of $\boldsymbol{\alpha}, \boldsymbol{\beta}$, and $\boldsymbol{\gamma}$ are solid, dashed, and dotted arcs, respectively. Components of $\mathbf{x}, \mathbf{y}$, and $\mathbf{w}$ are dark gray, white, and light gray dots, respectively.

The reader can verify that $\mu(\psi)=0$ in either case (in the second, it will help to split the obtuse hexagonal component of the domain as seen in Figure 1).

\subsubsection{3-gons and 4-gons in Heegaard moves.}

Definition 2.1.6. Let $\left(\Sigma ; \boldsymbol{\alpha} ; \boldsymbol{\beta} ; \boldsymbol{\beta}^{\prime} ; z\right)$ be a pointed Heegaard triple-diagram.

(i) Let $\beta_{j}^{\prime}$ differ from $\beta_{j}$ by an isotopy (avoiding $z$ ) such that $\beta_{j}^{\prime}$ intersects $\beta_{j}$ transversely in two canceling points and $\beta_{j} \cap \beta_{i}^{\prime}=\emptyset$ when $i \neq j$. Then we say that $\boldsymbol{\beta}^{\prime}$ differs from $\boldsymbol{\beta}$ by a pointed isotopy. A pointed isotopy, which preserves the set of intersection points $\mathbb{T}_{\boldsymbol{\alpha}} \cap \mathbb{T}_{\boldsymbol{\beta}}$ in the obvious way will be called a small pointed isotopy. 
(ii) Instead let $\beta_{1}, \beta_{2}$, and $\beta_{1}^{\prime}$ bound an embedded pair of pants disjoint from $z$ such that $\beta_{1}^{\prime}$ intersects $\beta_{1}$ transversely in two points. Assume also that $\beta_{j} \cap \beta_{i}^{\prime}$ for $i \neq j$, and that for $i>1, \beta_{i}^{\prime}$ relates to $\beta_{i}$ as $(i)$ above. Then we say that $\boldsymbol{\beta}^{\prime}$ differs from $\boldsymbol{\beta}$ by a pointed handleslide.

In either of the cases above, $\left(\Sigma ; \boldsymbol{\beta} ; \boldsymbol{\beta}^{\prime} ; z\right)$ is an admissible pointed Heegaard diagram for $\#^{g}\left(S^{1} \times S^{2}\right)$ and there is a canonical intersection point $\boldsymbol{\theta}_{\boldsymbol{\beta} \boldsymbol{\beta}^{\prime}} \in$ $\mathbb{T}_{\boldsymbol{\beta}} \cap \mathbb{T}_{\boldsymbol{\beta}^{\prime}}$ representing the top-degree homology class in $\widehat{H F}\left(Y_{\boldsymbol{\beta} \boldsymbol{\beta}^{\prime}}\right)$. If the triple-diagram is admissible, we have a well-defined chain map

$$
\widehat{f}_{\alpha \beta \beta^{\prime}}\left(\cdot \otimes \boldsymbol{\theta}_{\boldsymbol{\beta} \boldsymbol{\beta}^{\prime}}\right): \widehat{C F}\left(\mathcal{H}_{\boldsymbol{\alpha} \boldsymbol{\beta}}\right) \rightarrow \widehat{C F}\left(\mathcal{H}_{\boldsymbol{\alpha} \boldsymbol{\beta}^{\prime}}\right) .
$$

Note that in the original proof of invariance in $[\mathbf{1 3}]$, isotopies were not treated in terms of chain maps, which count pseudo-holomorphic 3-gons. Lipshitz proves in Proposition 11.4 from [8] that this can be done.

Now let $\left(\Sigma ; \boldsymbol{\alpha} ; \boldsymbol{\beta} ; \boldsymbol{\beta}^{\prime} ; \widetilde{\boldsymbol{\beta}}\right)$ be an admissible pointed Heegaard quadruplediagram, where $\widetilde{\boldsymbol{\beta}}$ differs from $\boldsymbol{\beta}$ by a small pointed isotopy, and $\boldsymbol{\beta}^{\prime}$ differs from $\boldsymbol{\beta}$ (and necessarily from $\widetilde{\boldsymbol{\beta}}$ ) by a pointed handleslide or a pointed isotopy. We can identify $\mathbb{T}_{\boldsymbol{\alpha}} \cap \mathbb{T}_{\boldsymbol{\beta}}$ with $\mathbb{T}_{\boldsymbol{\alpha}} \cap \mathbb{T}_{\widetilde{\boldsymbol{\beta}}}$ via the canonical nearestneighbor map $N_{\boldsymbol{\beta} \widetilde{\boldsymbol{\beta}}}: \mathbf{x} \mapsto \widetilde{\mathbf{x}}$, and extend this linearly to a chain complex isomorphism $N_{\boldsymbol{\beta} \widetilde{\boldsymbol{\beta}}}: \widehat{C F}\left(\mathcal{H}_{\boldsymbol{\alpha} \boldsymbol{\beta}}\right) \rightarrow \widehat{C F}\left(\mathcal{H}_{\boldsymbol{\alpha} \widetilde{\boldsymbol{\beta}}}\right)$ (note that $\left.\left(N_{\boldsymbol{\beta} \widetilde{\boldsymbol{\beta}}}\right) 6-1=N_{\widetilde{\boldsymbol{\beta}} \boldsymbol{\beta}}\right)$. We then have that

$$
\begin{aligned}
& \widehat{f}_{\boldsymbol{\alpha} \boldsymbol{\beta} \widetilde{\boldsymbol{\beta}}}\left(\mathbf{x} \otimes \widehat{f}_{\boldsymbol{\beta} \boldsymbol{\beta}^{\prime} \widetilde{\boldsymbol{\beta}}}\left(\boldsymbol{\theta}_{\boldsymbol{\beta} \boldsymbol{\beta}^{\prime}} \otimes \boldsymbol{\theta}_{\boldsymbol{\beta}^{\prime} \widetilde{\boldsymbol{\beta}}}\right)\right) \\
& \quad=\widehat{f}_{\boldsymbol{\alpha} \boldsymbol{\beta} \widetilde{\boldsymbol{\beta}}}\left(\mathbf{x} \otimes \boldsymbol{\theta}_{\boldsymbol{\beta} \widetilde{\boldsymbol{\beta}}}\right)=\widetilde{\mathbf{x}} \text { for all } \mathbf{x} \in \mathbb{T}_{\boldsymbol{\alpha}} \cap \mathbb{T}_{\boldsymbol{\beta}},
\end{aligned}
$$

where the last equality is due to Lemma 9.28 of [6] (cf. Proposition 9.8 of $[\mathbf{1 3}])$. Then by Theorem 2.1.3, we have that

$$
\begin{aligned}
& \widehat{f}_{\boldsymbol{\alpha} \boldsymbol{\beta}^{\prime} \widetilde{\boldsymbol{\beta}}}\left(\widehat{f}_{\boldsymbol{\alpha} \boldsymbol{\beta} \boldsymbol{\beta}^{\prime}}\left(\mathbf{x} \otimes \boldsymbol{\theta}_{\boldsymbol{\beta} \boldsymbol{\beta}^{\prime}}\right) \otimes \boldsymbol{\theta}_{\boldsymbol{\beta}^{\prime} \widetilde{\boldsymbol{\beta}}}\right)-\widetilde{\mathbf{x}} \\
& \quad=\widehat{\partial}\left(\widehat{h}_{\boldsymbol{\alpha} \boldsymbol{\beta} \boldsymbol{\beta}^{\prime} \widetilde{\boldsymbol{\beta}}}\left(\mathbf{x} \otimes \boldsymbol{\theta}_{\boldsymbol{\beta} \boldsymbol{\beta}^{\prime}} \otimes \boldsymbol{\theta}_{\boldsymbol{\beta}^{\prime} \widetilde{\boldsymbol{\beta}}}\right)\right)+\widehat{h}_{\boldsymbol{\alpha} \boldsymbol{\beta} \boldsymbol{\beta}^{\prime} \widetilde{\boldsymbol{\beta}}}\left(\widehat{\partial}\left(\mathbf{x} \otimes \boldsymbol{\theta}_{\boldsymbol{\beta} \boldsymbol{\beta}^{\prime}} \otimes \boldsymbol{\theta}_{\boldsymbol{\beta}^{\prime} \widetilde{\boldsymbol{\beta}}}\right)\right) .
\end{aligned}
$$

Letting $\widetilde{\boldsymbol{\beta}}^{\prime}$ differ from $\boldsymbol{\beta}^{\prime}$ by a pointed isotopy and studying the admissible pointed Heegaard quadruple-diagram $\left(\Sigma ; \boldsymbol{\alpha} ; \boldsymbol{\beta}^{\prime} ; \boldsymbol{\beta} ; \widetilde{\boldsymbol{\beta}}^{\prime} ; z\right)$, one finds that for $\mathbf{x} \in \mathbb{T}_{\boldsymbol{\alpha}} \cap \mathbb{T}_{\boldsymbol{\beta}^{\prime}}$

$$
\begin{aligned}
& \widehat{f}_{\boldsymbol{\alpha} \boldsymbol{\beta} \widetilde{\boldsymbol{\beta}}^{\prime}}\left(\widehat{f}_{\boldsymbol{\alpha} \boldsymbol{\beta}^{\prime} \boldsymbol{\beta}}\left(\mathbf{x} \otimes \boldsymbol{\theta}_{\boldsymbol{\beta}^{\prime} \boldsymbol{\beta}}\right) \otimes \boldsymbol{\theta}_{\boldsymbol{\beta} \widetilde{\boldsymbol{\beta}}^{\prime}}\right)-\widetilde{\mathbf{x}} \\
& \quad=\widehat{\partial}\left(\widehat{h}_{\boldsymbol{\alpha} \boldsymbol{\beta}^{\prime} \boldsymbol{\beta} \widetilde{\boldsymbol{\beta}}^{\prime}}\left(\mathbf{x} \otimes \boldsymbol{\theta}_{\boldsymbol{\beta}^{\prime} \boldsymbol{\beta}} \otimes \boldsymbol{\theta}_{\boldsymbol{\beta} \widetilde{\boldsymbol{\beta}}^{\prime}}\right)\right)+\widehat{h}_{\boldsymbol{\alpha} \boldsymbol{\beta}^{\prime} \boldsymbol{\beta} \widetilde{\boldsymbol{\beta}}^{\prime}}\left(\widehat{\partial}\left(\mathbf{x} \otimes \boldsymbol{\theta}_{\boldsymbol{\beta}^{\prime} \boldsymbol{\beta}} \otimes \boldsymbol{\theta}_{\boldsymbol{\beta} \widetilde{\boldsymbol{\beta}}^{\prime}}\right)\right) .
\end{aligned}
$$

Therefore, we see that when $\boldsymbol{\beta}^{\prime}$ differs from $\boldsymbol{\beta}$ by a pointed isotopy or a pointed handleslide, the chain map $\widehat{f}_{\alpha \beta \beta^{\prime}}\left(\cdot \otimes \boldsymbol{\theta}_{\boldsymbol{\beta} \boldsymbol{\beta}^{\prime}}\right)$ is a chain homotopy 
equivalence with homotopy inverse given by $\widehat{f}_{\alpha \beta^{\prime} \beta}\left(\cdot \otimes \boldsymbol{\theta}_{\boldsymbol{\beta}^{\prime} \boldsymbol{\beta}}\right)$. Furthermore, the associated homotopies relating their compositions to the appropriate identity maps are given by

$$
\begin{aligned}
& N_{\widetilde{\boldsymbol{\beta} \beta}} \circ \widehat{h}_{\boldsymbol{\alpha} \boldsymbol{\beta} \boldsymbol{\beta}^{\prime} \widetilde{\boldsymbol{\beta}}}\left(\cdot \otimes \boldsymbol{\theta}_{\boldsymbol{\beta} \boldsymbol{\beta}^{\prime}} \otimes \boldsymbol{\theta}_{\boldsymbol{\beta}^{\prime} \widetilde{\boldsymbol{\beta}}}\right) \text { and } \\
& N_{\widetilde{\boldsymbol{\beta}}^{\prime} \boldsymbol{\beta}^{\prime}} \circ \widehat{h}_{\boldsymbol{\alpha} \boldsymbol{\beta}^{\prime} \boldsymbol{\beta} \widetilde{\boldsymbol{\beta}}^{\prime}}\left(\mathbf{x} \otimes \boldsymbol{\theta}_{\boldsymbol{\beta}^{\prime} \boldsymbol{\beta}} \otimes \boldsymbol{\theta}_{\left.\boldsymbol{\beta}_{\widetilde{\boldsymbol{\beta}}^{\prime}}\right)} .\right.
\end{aligned}
$$

Remark 2.1.7. Given admissible pointed Heegaard quadruple-diagrams $\left(\Sigma ; \widetilde{\boldsymbol{\alpha}} ; \boldsymbol{\alpha}^{\prime} ; \boldsymbol{\alpha} ; \boldsymbol{\beta} ; z\right)$ and $\left(\Sigma ; \widetilde{\boldsymbol{\alpha}}^{\prime} ; \boldsymbol{\alpha} ; \boldsymbol{\alpha}^{\prime} ; \boldsymbol{\beta} ; z\right)$, where $\boldsymbol{\alpha}^{\prime}$ differs from $\boldsymbol{\alpha}$ by a pointed isotopy or a pointed handleslide (with $\widetilde{\boldsymbol{\alpha}}$ and $\widetilde{\boldsymbol{\alpha}}^{\prime}$ analogous to $\widetilde{\boldsymbol{\beta}}$ and $\widetilde{\boldsymbol{\beta}}^{\prime}$, , one can define the chain maps $\widehat{f}_{\boldsymbol{\alpha}^{\prime} \boldsymbol{\alpha} \boldsymbol{\beta}}\left(\boldsymbol{\theta}_{\boldsymbol{\alpha}^{\prime} \boldsymbol{\alpha}} \otimes \cdot\right)$ and $\widehat{f}_{\boldsymbol{\alpha} \boldsymbol{\alpha}^{\prime} \boldsymbol{\beta}}\left(\boldsymbol{\theta}_{\boldsymbol{\alpha} \boldsymbol{\alpha}^{\prime}} \otimes \cdot\right)$. These two maps are chain homotopy inverses to one another, and the associated chain homotopies are

$$
\begin{aligned}
& N_{\widetilde{\boldsymbol{\alpha}} \boldsymbol{\alpha}} \circ \widehat{h}_{\widetilde{\boldsymbol{\alpha}} \boldsymbol{\alpha}^{\prime} \boldsymbol{\alpha} \boldsymbol{\beta}}\left(\boldsymbol{\theta}_{\widetilde{\boldsymbol{\alpha}} \boldsymbol{\alpha}^{\prime}} \otimes \boldsymbol{\theta}_{\boldsymbol{\alpha}^{\prime} \boldsymbol{\alpha}} \otimes \cdot\right) \quad \text { and } \\
& N_{\widetilde{\boldsymbol{\alpha}}^{\prime} \boldsymbol{\alpha}^{\prime}} \circ \widehat{h}_{\widetilde{\boldsymbol{\alpha}}^{\prime} \boldsymbol{\alpha} \boldsymbol{\alpha}^{\prime} \boldsymbol{\beta}}\left(\boldsymbol{\theta}_{\widetilde{\boldsymbol{\alpha}}^{\prime} \boldsymbol{\alpha}} \otimes \boldsymbol{\theta}_{\boldsymbol{\alpha} \boldsymbol{\alpha}^{\prime}} \otimes \cdot\right) .
\end{aligned}
$$

2.2. Periodic domains. Recall that a periodic domain in a pointed Heegaard diagram $(\Sigma ; \boldsymbol{\alpha} ; \boldsymbol{\beta} ; z)$ is a domain avoiding the basepoint $z$ whose boundary is a sum of the $\boldsymbol{\alpha}$ and $\boldsymbol{\beta}$ circles. Denote by $\Pi_{\boldsymbol{\alpha} \boldsymbol{\beta}} \subset H_{2}(\Sigma ; \mathbb{Z})$ the group of such periodic domains and let $S_{\boldsymbol{\alpha} \boldsymbol{\beta}}=S_{\boldsymbol{\alpha}}+S_{\boldsymbol{\beta}} \subset H_{1}(\Sigma ; \mathbb{Z})$ be the span of the $\boldsymbol{\alpha}$ and $\boldsymbol{\beta}$ circles.

Recall also the analogous notions of triply- and quadruply-periodic domains in Heegaard triple-diagrams and quadruple-diagrams.

In $[\mathbf{1 0}]$, it is shown that if $(\Sigma ; \boldsymbol{\alpha} ; \boldsymbol{\beta} ; z)$ is a pointed Heegaard diagram, then $\Pi_{\boldsymbol{\alpha} \boldsymbol{\beta}}$ is a free Abelian group of $\operatorname{rank} 2 g-\operatorname{rank}\left(S_{\boldsymbol{\alpha} \boldsymbol{\beta}}\right)$. It can be shown in a completely analogous way that for a triple-diagram (respectively, quadruplediagram), the group $\Pi_{\boldsymbol{\alpha} \boldsymbol{\beta} \boldsymbol{\gamma}}$ (respectively $\Pi_{\boldsymbol{\alpha} \boldsymbol{\beta} \boldsymbol{\gamma} \boldsymbol{\delta}}$ ) is free Abelian of rank $3 g-\operatorname{rank}\left(S_{\boldsymbol{\alpha} \boldsymbol{\beta} \boldsymbol{\gamma}}\right)$ (respectively, $\left.4 g-\operatorname{rank}\left(S_{\boldsymbol{\alpha} \boldsymbol{\beta} \boldsymbol{\gamma} \boldsymbol{\delta}}\right)\right)$. One should note that because we do not permit periodic domains in a pointed Heegaard diagram to intersect the basepoint, our ranks are 1 lower than those stated in [10].

Let $\boldsymbol{\alpha}$ and $\boldsymbol{\beta}$ be two $g$-tuples of attaching circles on a genus- $g$ surface $\Sigma$ such that $\boldsymbol{\beta}$ differs from $\boldsymbol{\alpha}$ by a pointed isotopy. Then for each $i$, the circles $\alpha_{i}$ and $\beta_{i}$ are separated by two 2 -sided regions, and we denote by $\mathcal{D}_{i}^{\boldsymbol{\alpha} \boldsymbol{\beta}}$ the periodic domain which is their difference - these domains look like the ones shown in Figure 2(a).

Now instead let $\boldsymbol{\alpha}$ and $\boldsymbol{\beta}$ be two $g$-tuples of attaching circles on a genus- $g$ surface $\Sigma$ such that $\boldsymbol{\beta}$ differs from $\boldsymbol{\alpha}$ by a pointed handleslide of $\alpha_{1}$ over $\alpha_{2}$. For $i>1$, the circles $\alpha_{i}$ and $\beta_{i}$ are separated by two thin 2-sided regions, and we denote by $\mathcal{D}_{i}^{\boldsymbol{\alpha} \boldsymbol{\beta}}$ the periodic domain which is their difference. The circles $\alpha_{1}$ and $\beta_{1}$ are separated by a thin 2 -sided region, and we denote by $\mathcal{D}_{1}^{\boldsymbol{\alpha} \boldsymbol{\beta}}$ the periodic domain, which is the difference between this region and 

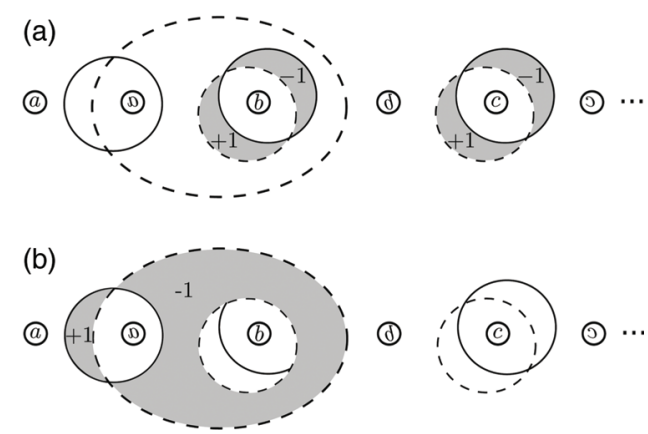

Figure 2. The periodic domains $\mathcal{D}_{i}^{\boldsymbol{\alpha} \boldsymbol{\beta}}$ for the handleslide of $\alpha_{1}$ over $\alpha_{2}$. The $\boldsymbol{\alpha}$ circles are solid and the $\boldsymbol{\beta}$ circles are dashed. The domains of interest are shaded and local coefficients are labelled. (a) The handleslide domains $\mathcal{D}_{i}^{\boldsymbol{\alpha} \boldsymbol{\beta}}$ for $i>1$ and (b) The handleslide domain $\mathcal{D}_{1}^{\boldsymbol{\alpha} \boldsymbol{\beta}}$.

the annular region bounded by $\alpha_{1}, \alpha_{2}$, and $\beta_{1}$. These domains can be seen in Figure 2.

The following facts are exercises in linear algebra:

Proposition 2.2.1. Let $(\Sigma ; \boldsymbol{\alpha} ; \boldsymbol{\beta} ; z)$ be a pointed Heegaard diagram of genus $g$ such that $\boldsymbol{\beta}$ is obtained from $\boldsymbol{\alpha}$ via a pointed isotopy or pointed handleslide. Then the set $\left\{\mathcal{D}_{1}^{\boldsymbol{\alpha} \boldsymbol{\beta}}, \ldots, \mathcal{D}_{g}^{\boldsymbol{\alpha} \boldsymbol{\beta}}\right\}$ is a generating set for the group $\Pi_{\boldsymbol{\alpha} \boldsymbol{\beta}}$.

Proposition 2.2.2. Let $(\Sigma ; \boldsymbol{\alpha} ; \boldsymbol{\beta} ; \boldsymbol{\gamma} ; z)$ be a pointed Heegaard triple-diagram of genus $g$ such that $\boldsymbol{\gamma}$ is obtained from $\boldsymbol{\beta}$ via a pointed isotopy or pointed handleslide. Then the set $\left\{\mathcal{D}_{1}^{\boldsymbol{\beta} \gamma}, \ldots, \mathcal{D}_{g}^{\boldsymbol{\beta} \gamma}\right\}$ is a generating set for the group $\Pi_{\boldsymbol{\alpha} \boldsymbol{\beta} \gamma}$.

Proposition 2.2.3. Let $(\Sigma ; \boldsymbol{\alpha} ; \boldsymbol{\beta} ; \boldsymbol{\gamma} ; \boldsymbol{\delta} ; z)$ be a pointed Heegaard quadruplediagram of genus $\mathrm{g}$ such that $\boldsymbol{\gamma}$ is obtained from $\boldsymbol{\beta}$ via a pointed isotopy or pointed handleslide, and $\boldsymbol{\delta}$ is obtained from $\boldsymbol{\beta}$ via a small pointed isotopy. Then the set $\left\{\mathcal{D}_{1}^{\boldsymbol{\beta} \gamma}, \ldots, \mathcal{D}_{g}^{\boldsymbol{\beta} \gamma}\right\} \cup\left\{\mathcal{D}_{1}^{\boldsymbol{\beta} \boldsymbol{\delta}}, \ldots, \mathcal{D}_{g}^{\boldsymbol{\beta} \boldsymbol{\delta}}\right\}$ is a generating set for the group $\Pi_{\boldsymbol{\alpha} \boldsymbol{\beta} \gamma \boldsymbol{\delta}}$.

The above facts imply the following useful fact about admissibility of multi-diagrams:

Proposition 2.2.4. Let $(\Sigma ; \boldsymbol{\alpha} ; \boldsymbol{\beta} ; \boldsymbol{\gamma} ; \boldsymbol{\delta} ; z)$ be a pointed Heegaard quadruplediagram of genus $g$ such that $\boldsymbol{\gamma}$ is obtained from $\boldsymbol{\beta}$ via a pointed isotopy or pointed handleslide, and $\boldsymbol{\delta}$ is obtained from $\boldsymbol{\beta}$ via a small pointed isotopy. Then if the six pointed diagrams formed by choosing any two tuples out of $\boldsymbol{\alpha}$, $\boldsymbol{\beta}, \boldsymbol{\gamma}$, and $\boldsymbol{\delta}$ are all admissible, so is the quadruple-diagram. Moreover, each of the four triple-diagrams composed of three of the tuples is also admissible. 
Proof. Let $\mathcal{D}_{0}, \mathcal{D}_{1}, \ldots, \mathcal{D}_{N}$ denote the connected components of

$$
\Sigma \backslash\left(\cup_{i} \alpha_{i}\right) \backslash\left(\cup_{i} \beta_{i}\right) \backslash\left(\cup_{i} \gamma_{i}\right) \backslash\left(\cup_{i} \delta_{i}\right),
$$

where $\mathcal{D}_{0}$ is the component containing $z$. Consider some nontrivial quadruply-periodic domain

$$
\mathcal{P}=\sum_{j=1}^{N} c_{j} \mathcal{D}_{j}
$$

Then by Proposition 2.2.3, we can write

$$
\mathcal{P}=\sum_{j=1}^{g} n_{j} \mathcal{D}_{j}^{\boldsymbol{\beta} \boldsymbol{\gamma}}+\sum_{j=1}^{g} m_{j} \mathcal{D}_{j}^{\boldsymbol{\beta} \boldsymbol{\delta}} .
$$

Now at least one of $n_{1}, \ldots, n_{g}, m_{1}, \ldots, m_{g}$ is nonzero - without loss of generality, let it be $n_{1}$. Now the domain $\mathcal{D}_{1}^{\boldsymbol{\beta} \gamma}$ is the sum of two regions, which have coefficients +1 and -1 , respectively. Neither can be canceled by any other terms in the right-hand side of equation (2.3), and so there are both positive and negative numbers among the $c_{i}$.

The argument for triple diagrams is similar, making use of Proposition 2.2 .2 .

2.3. Filtrations and spectral sequences. Let $\left(\mathcal{C}_{*}, \partial\right)$ be a chain complex generated by $\left\{x_{i}\right\}_{i=1}^{n}$ and equipped with a filtration grading $f:\left\{x_{i}\right\} \rightarrow \mathbb{Z}$. We can view the filtration as the nested family of subcomplexes $\left\{\mathcal{F}_{k}\right\}_{k \in \mathbb{Z}}$, with

$$
\mathcal{F}_{k}=\operatorname{span}\left\{x_{i}: f\left(x_{i}\right) \leq k\right\} .
$$

Definition 2.3.1. Let $(\mathcal{C}, \partial)$ and $\left(\mathcal{C}^{\prime}, \partial^{\prime}\right)$ be chain complexes with filtrations $\left\{\mathcal{F}_{k}\right\}$ and $\left\{\mathcal{F}_{k}^{\prime}\right\}$.

(a) A chain map $F:(\mathcal{C}, \partial) \rightarrow\left(\mathcal{C}^{\prime}, \partial^{\prime}\right)$ is called a filtered chain map if for all $k, F\left(\mathcal{F}_{k}\right) \subset \mathcal{F}_{k}^{\prime}$.

(b) Let $H:(\mathcal{C}, \partial) \rightarrow\left(\mathcal{C}^{\prime}, \partial^{\prime}\right)$ be a chain homotopy connecting two maps $F, G:(\mathcal{C}, \partial) \rightarrow\left(\mathcal{C}^{\prime}, \partial^{\prime}\right)$. We call $H$ a filtered chain homotopy if for all $k$, $H\left(\mathcal{F}_{k}\right) \subset \mathcal{F}_{(k+1)}^{\prime}$

(c) Let $F:(\mathcal{C}, \partial) \rightarrow\left(\mathcal{C}^{\prime}, \partial^{\prime}\right)$ be a chain homotopy equivalence with homotopy inverse map $G:\left(\mathcal{C}^{\prime}, \partial^{\prime}\right) \rightarrow(\mathcal{C}, \partial)$ and associated homotopies $H:(\mathcal{C}, \partial) \rightarrow$ $(\mathcal{C}, \partial)$ from $G \circ F$ to $i d_{\mathcal{C}}$ and $H^{\prime}:\left(\mathcal{C}^{\prime}, \partial^{\prime}\right) \rightarrow\left(\mathcal{C}^{\prime}, \partial^{\prime}\right)$ from $F \circ G$ to $i d_{\mathcal{C}^{\prime}}$. We say that $F$ is a filtered chain homotopy equivalence if both $F$ and $G$ are filtered maps and both $H$ and $H^{\prime}$ are filtered chain homotopies.

For each $i, k \in \mathbb{Z}$, let $\mathcal{F}_{k} \mathcal{C}_{i}:=\mathcal{F}_{k} \cap \mathcal{C}_{i}$. Now note that the filtration on $\mathcal{C}_{*}$ induces a filtration on the homology of $\mathcal{C}_{*}$ given by

$$
\mathcal{F}_{k} H_{i}\left(\mathcal{C}_{*}\right):=\left\{\alpha \in H_{i}\left(\mathcal{C}_{*}\right) \mid \alpha=[x] \text { for some } x \in \mathcal{F}_{k} \mathcal{C}_{i}\right\} .
$$


One can associate to a filtered complex a spectral sequence, which is defined recursively. First, for each $p, q \in \mathbb{Z}$, define the associated graded module by

$$
E_{p, q}^{0}:=\mathcal{F}_{p} \mathcal{C}_{p+q} / \mathcal{F}_{p-1} \mathcal{C}_{p+q}
$$

The differential $\partial$ induces a differential $\partial_{0}: E_{p, q}^{0} \rightarrow E_{p, q-1}^{0}$, and we refer to the chain complex $\left(E^{0}, \partial_{0}\right)$ as the $E^{0}$-page of the spectral sequence. The homology of this associated graded complex is denoted by

$$
E_{p, q}^{1}:=H_{p+q}\left(\mathcal{F}_{p} \mathcal{C}_{*} / \mathcal{F}_{p-1} \mathcal{C}_{*}\right)
$$

and $\partial$ induces a differential $\partial_{1}: E_{p, q}^{1} \rightarrow E_{p-1, q}^{1}$ (yielding the $E^{1}$-page $\left.\left(E^{1}, \partial_{1}\right)\right)$.

Continuing this process, one obtains a sequence of chain complexes $\left(E^{k}, \partial_{k}\right)$ (the $E^{k}$-pages), where $\partial_{k}: E_{p, q}^{k} \rightarrow E_{p-k, q+k-1}^{k}$ and

$$
E_{p, q}^{k}:=\frac{\operatorname{Ker}\left(\partial_{k}: E_{p, q}^{k} \rightarrow E_{p-k, q+k-1}^{k}\right)}{\operatorname{Im}\left(\partial_{k}: E_{p-r, q-r+1}^{r} \rightarrow E_{p, q}^{k}\right)} .
$$

Since $\mathcal{C}_{*}$ was finitely-generated, eventually these pages stabilize and are isomorphic to the homology of $\mathcal{C}_{*}$. More precisely, for $k$ sufficiently large,

$$
E_{p, q}^{k} \cong \mathcal{F}_{p} H_{p+q}\left(\mathcal{C}_{*}\right) / \mathcal{F}_{p-1} H_{p+q}\left(\mathcal{C}_{*}\right) \quad \text { and } \quad \partial_{k} \equiv 0
$$

If $K$ denotes the smallest such $k$ such that the above holds, we say that the spectral sequence collapses at $E^{K}$.

One should note that the spectral sequence will collapse at $E^{1}$ if $\partial$ preserves the filtration, i.e., if for each $j$,

$$
\partial\left(x_{j}\right)=\sum_{f\left(x_{i}\right)=f\left(x_{j}\right)} a_{i} x_{i} .
$$

\section{Braids and the Bigelow picture}

Let $B_{2 n}$ denote the braid group on $2 n$ strands. This group is generated by $\left\{\sigma_{1}, \ldots, \sigma_{2 n-1}\right\}$, where $\sigma_{k}$ denotes a half-twist of the $k^{\text {th }}$ strand over the $(k+1)^{s t}$ strand. Given a braid $b \in B_{2 n}$, we can obtain a diagram of a knot or a link (the plat closure of $b$ ) by connecting ends of consecutive strands with segments at the top and bottom, as shown in Figure 3.

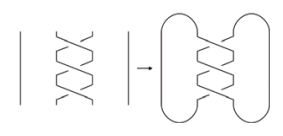

Figure 3. The left-handed trefoil. 


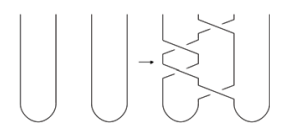

Figure 4. The Birman move $b \mapsto b B$.

Any knot $K$ can be presented as the plat closure of an element in $B_{2 n}$. Many distinct braid elements can have isotopic plat closures, but such braids are related.

Definition 3.0.2. Let $K_{2 n}$ be the subgroup of the braid group $B_{2 n}$ generated by $A=\sigma_{1}, B=\sigma_{2} \sigma_{1}^{2} \sigma_{2}$, and $C_{i}=\sigma_{2 i} \sigma_{2 i-1} \sigma_{2 i+1} \sigma_{2 i}$ for $i=1,2, \ldots, 2 n$.

Theorem 3.0.3 (Theorem 1 from [4]). Let $b \in B_{2 n}$ and $b^{\prime} \in B_{2 n^{\prime}}$ be two oriented braids. The braids $b$ and $b^{\prime}$ have isotopic plat closures if and only if they are related by a finite sequence of the following moves:

(i) $b \mapsto g b h$ where $b \in B_{2 n}$ and $g, h \in K_{2 n}$

(ii) $b \leftrightarrow \sigma_{2 n} b$ where $b \in B_{2 n}$ and $\sigma_{2 n} b \in B_{2 n+2}$

We will refer to the local moves appearing in the statement of Theorem 3.0.3 as "Birman moves" - one of these is illustrated in Figure 4.

3.1. The Bigelow generators. Let $D_{2 n} \subset \mathbb{C}$ denote the unit disk with $2 n$ punctures $\mu_{1}, \ldots, \mu_{2 n}$ evenly spaced along $\mathbb{R} \cap D$. We can view the braid group $B_{2 n}$ as the mapping class group of $D_{2 n}$, where the generator $\sigma_{k}$ is a diffeomorphism, which is the identity outside of a neighborhood of the $k^{\text {th }}$ and $(k+1)^{\text {st }}$ punctures and exchanges these two punctures by a counterclockwise half-twist. Any braid can be written as a word in the $\sigma_{k}^{\prime} s$, and we view them as operating on $D_{2 n}$ in this way, read from left to right.

Let $b \in B_{2 n}$ be an oriented braid on $2 n$ strands. We will establish some terminology, following Bigelow in [3].

Definition 3.1.1. Let $D \subset \mathbb{C}$ be the unit disk.

(i) Let the standard fork diagram in $D_{2 n}$ be a collection of maps $\alpha_{1}, \ldots, \alpha_{n}: I \rightarrow D$ and $h_{1}, \ldots, h_{n}: I \rightarrow D$ called tine edges and handles, respectively, such that the following hold:

(a) The segments $\left.\alpha_{i}\right|_{(0,1)}$ are disjoint embeddings of $(0,1)$ into $D_{2 n}$ such that for each $k, \alpha_{k}(0)=\mu_{2 k-1}, \alpha_{k}(1)=\mu_{2 k}$, and $\alpha_{k}(t) \in \mathbb{R}$ for all $t \in I$.

(b) The segments $\left.h_{i}\right|_{(0,1)}$ are disjoint embeddings of $(0,1)$ into $D_{2 n}$ such that that for each $k, h_{k}(1)=d_{k} \in \partial D, h_{k}(0)=m_{k}$ is the midpoint of the segment $\alpha_{k}$, and the segment $h_{k}$ is vertical.

(ii) Let a fork diagram for $b$ be the standard fork diagram along with the compositions $b \circ \alpha_{1}, \ldots, b \circ \alpha_{n}$ and $b \circ h_{1}, \ldots, b \circ h_{n}$. We will let $\beta_{k}=b \circ \alpha_{k}$. 


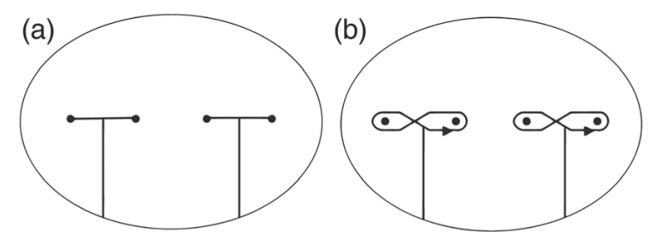

Figure 5. Structures in fork diagrams (a) Tine edges $\alpha_{k}$ and (b) Figure-eights $E_{k}$.

(iii) Let an augmented fork diagram for $b$ be obtained from a fork diagram by replacing each arc $\beta_{k}$ with $b E_{k}$, where $E_{k}$ is a figure-eight encircling $\mu_{2 k-1}$ and $\mu_{2 k}$, where $E_{k}$ is oriented such that it winds counterclockwise about $\mu_{2 k}$.

A standard fork diagram in $D_{4}$ and an augmented version of it are depicted in Figure 5.

The reader should note that by drawing a picture containing just the $\alpha$ and $\beta$ arcs and treating the $\alpha$ arcs as undercrossings at each intersection, we get a diagram of the plat closure of $b$.

We will define some notation. Let $\operatorname{Conf}^{n}(\mathbb{C})$ denote the configuration space of $\mathbb{C}$, i.e., the set of unordered $n$-tuples of distinct points in $\mathbb{C}$. Let $\widetilde{\mathcal{Z}}$ be the set of intersections between $\alpha$ and $\beta$ arcs. Then if $\tau$ denotes the set of puncture points, we see that $\tau \subset \widetilde{\mathcal{Z}}$. Then we construct a set $\mathcal{Z}$ by doubling the points in $\widetilde{\mathcal{Z}} \backslash \tau$ by introducing for each $x \in \tau$ one element $e_{x} \in \mathcal{Z}$ and for each $x \in \widetilde{\mathcal{Z}} \backslash \tau$ two elements $e_{x}, e_{x}^{\prime} \in \mathcal{Z}$. The set $\mathcal{Z}$ can then be seen as the intersections points between $\alpha$ arcs and figure-eights $b E_{k}$. We distinguish between $e_{x}$ and $e_{x}^{\prime}$ by requiring that the loop traveling along a figure-eight from $e_{x}$ to $e_{x}^{\prime}$ and back to $e_{x}$ along an $\alpha$ arc has winding number +1 around the puncture point.

Remark 3.1.2. Via an abuse of notation, we will often refer to the points corresponding to $x \in \widetilde{\mathcal{Z}}$ as $x \in \mathcal{Z}$ (if $x \in \tau$ ) or $x, x^{\prime} \in \mathcal{Z}$ (if $x \in \widetilde{\mathcal{Z}} \backslash \tau$ ).

We then define $\widetilde{\mathcal{G}}=\left(\alpha_{1} \times \cdots \times \alpha_{n}\right) \cap\left(\beta_{1} \times \cdots \times \beta_{n}\right) \subset \operatorname{Conf}^{n}(\mathbb{C})$, the set of unordered $n$-tuples of points in $\widetilde{\mathcal{Z}}$ such that no two points are on the same $\alpha$ or $\beta$ arcs.

Similarly, define $\mathcal{G}=\left(\alpha_{1} \times \cdots \times \alpha_{n}\right) \cap\left(b E_{1} \times \cdots \times b E_{n}\right) \subset \operatorname{Conf}^{n}(\mathbb{C})$, which will be referred to as the set of Bigelow generators for the diagram.

Remark 3.1.3. From this point forward, something of the form $x y$ will denote an element in $\mathcal{G}$ or $\widetilde{\mathcal{G}}$ such that $x \in \mathcal{Z}$ or $x \in \widetilde{\mathcal{Z}}$ is some component of the $n$-tuple and $\mathbf{y}$ is the rest of the $n$-tuple.

3.2. Gradings on the Bigelow generators. We will define some gradings $Q, T, P: \mathcal{G} \rightarrow \mathbb{Z}$ based on loops in the configuration space of the disk. Our 


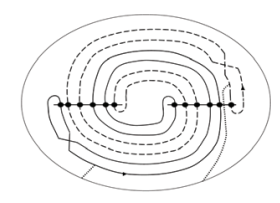

Figure 6. The augmented fork diagram induced by $\sigma_{2}^{3} \in B_{4}$.

Table 1. Distributions of $T, P^{*}, P, Q^{*}$, and $Q$ for $\sigma_{2}^{3} \in B_{4}$.

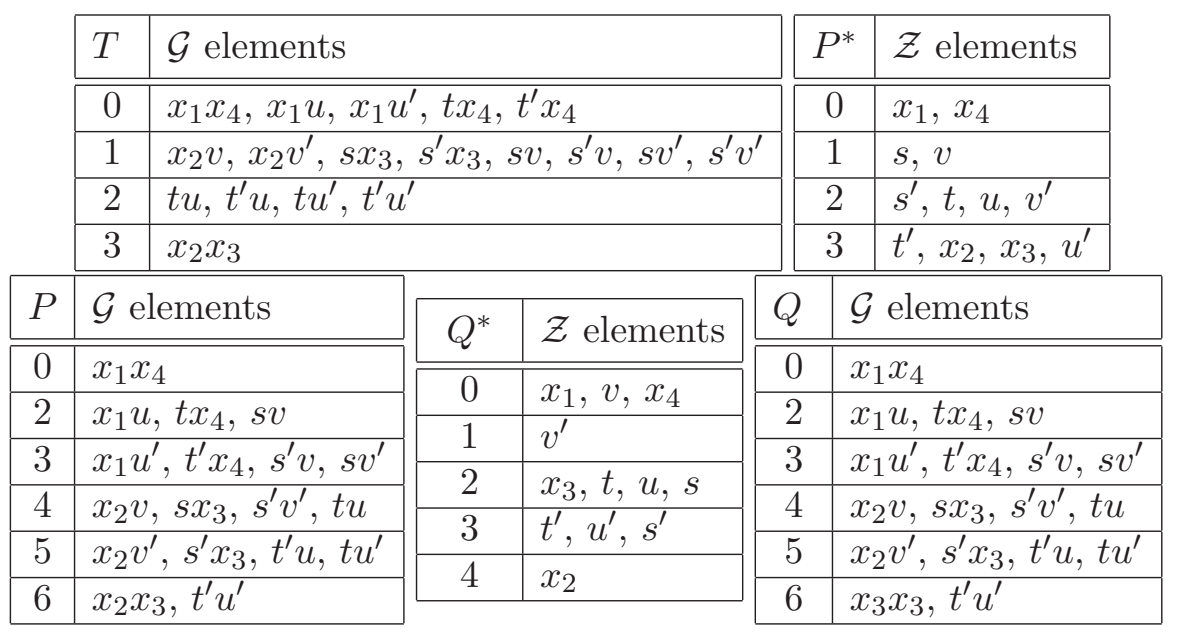

definitions of $Q$ and $T$ are identical to Bigelow's in [3], while our definition for $P$ is adapted from Manolescu's definition for $\widetilde{P}$ in $[\mathbf{9}]$ (in which he used braid closures).

For the sake of concreteness, a sample calculation will accompany the description of the gradings. We will study the left-handed trefoil knot depicted as the plat closure of $\sigma_{2}^{3} \in B_{4}$, as seen in Figure 3 .

Figure 6 depicts the augmented fork diagram for our example. Label the elements of $\mathcal{Z}$ from left to right in the diagram as $\mathcal{Z}=\left\{x_{1}, s, s^{\prime}, t, t^{\prime}, x_{2}, x_{3}, u^{\prime}\right.$, $\left.u, v^{\prime}, v, x_{4}\right\}$.

One can verify that the set of Bigelow generators is given by

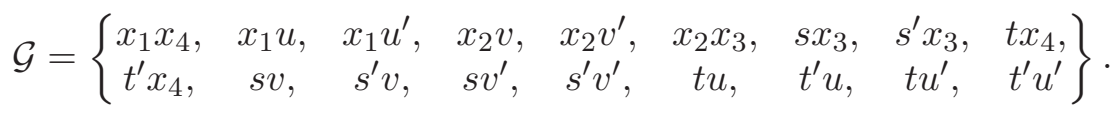

We will turn to defining various gradings on the set $\mathcal{G}$. Grading distributions for our trefoil example can be found in Table 1. Figure 7 illustrates how to compute the gradings in practice.

3.2.1. The $Q$ grading. The grading $Q$ on $\mathcal{G}$ will be computed additively from a grading $Q^{*}: \mathcal{Z} \rightarrow \mathbb{Z}$. Consider some $x \in \mathcal{Z}$, where $x \in \alpha_{i} \cap b E_{j}$. 
(a)

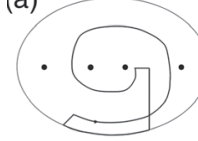

(b)

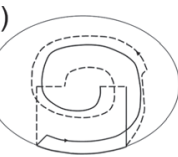

(c)

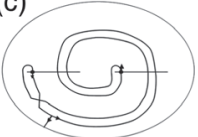

Figure 7. Computing the gradings $Q, T$, and $P$. (a) $Q^{*}\left(x_{3}\right)=2$, (b) $T\left(x_{2} x_{3}\right)=3$, (c) $P^{*}\left(x_{3}\right)=3$ and $P^{*}\left(x_{1}\right)=0$.

Define an arc $\gamma_{x}$ in the disk by starting at $d_{j}$, traveling along $-b h_{j}$ to $b m_{j}$, traveling along $b E_{j}$ to $x$, traveling along $\alpha_{i}$ to $m_{i}$, and traveling along $h_{i}$ to $d_{i}$. Then let $\gamma_{i j}$ be the arc traveling along the lower portion of $\partial D$ from $d_{i}$ to $d_{j}$. Then $\gamma_{x} \gamma_{i j}$ is an arc from $d_{j}$ to itself, and we define $Q^{*}(x)$ to be the winding number of this loop around the set of punctures.

Then for each $\mathbf{e}=e_{1} e_{2} \ldots e_{n} \in \mathcal{G}$, define

$$
Q(\mathbf{e})=\sum_{i=1}^{n} Q^{*}\left(e_{i}\right)
$$

3.2.2. The $\boldsymbol{T}$ grading. Given $\mathbf{e}=e_{1} e_{2} \cdots e_{n} \in \mathcal{G}$, we have that for each $k, e_{k}=e_{x_{k}}$ or $e_{k}=e_{x_{k}}^{\prime}$ for some $x_{k} \in \widetilde{\mathcal{Z}}$. Now let $\mathbf{x}=x_{1} x_{2} \cdots x_{n} \in \widetilde{\mathcal{G}}$.

Then denote by $\tilde{\gamma}_{x_{k}}$ the arc obtained by replacing the figure-eight segments of $\gamma_{x_{k}}$ with the corresponding $\beta$ arc segments. Then $T(\mathbf{x})$ can be computed as twice the sum of the pairwise winding of the $\tilde{\gamma}_{x_{k}}$ around each other. In other words, if $\tilde{\gamma}_{x_{k}}$ and $\tilde{\gamma}_{x_{m}}$ make a half-twist counter-clockwise around each other for $k \neq m$, this contributes +1 to the value of $T(\mathbf{x})$. Define $T: \mathcal{G} \rightarrow \mathbb{Z}$ by letting $T(\mathbf{e})=T(\mathbf{x})$.

3.2.3. The $\boldsymbol{P}$ grading. This grading will be computed additively from a grading $P^{*}: \mathcal{Z} \rightarrow \mathbb{Z}$, which measures twice the relative winding number of the tangent vectors to the figure eights $b E_{k}$ at the points in $\mathcal{Z}$.

For $x \in \mathcal{Z}$, where $x \in \alpha_{i} \cap b E_{j}$, we define $P^{*}(x)$ in the following way. We view the arc $b h_{j}$ as being oriented downward at the point where it intersects $\partial D$. Let $b E_{j}$ have the orientation induced by the orientation on $E_{j}$ in the standard fork diagram. Then we let $P^{*}(x)$ be twice the winding number of the tangent vector relative to the downward-pointing tangent vector at the point $b h_{j} \cap \partial D$. In other words, if the tangent vector makes $k$ counter-clockwise half-revolutions and $m$ clockwise half-revolutions as we travel first along $b h_{j}$ from $b h_{j}(0)$ to $b h_{j}(1)$ then along $b E_{j}$ to $x$, then we set $P^{*}(x)=m-k$. This number is an integer because we assume that at any point $x \in \mathcal{Z}$, the figure-eight intersects the $\alpha$ arc at a right angle.

Then for $\mathbf{e}=e_{1} e_{2} \cdots e_{n} \in \mathcal{G}$, we define

$$
P(\mathbf{e})=\sum_{i=1}^{n} P^{*}\left(e_{i}\right)
$$




\section{The anti-diagonal filtration}

We review here how one obtains from the above picture a filtration on the Heegaard Floer complex, following Manolescu in [9] and Seidel and Smith in $[\mathbf{2 2}]$.

We will first recall in Section 4.1 a formal construction involving graded totally-real submanifolds, as discussed by Manolescu in [9]. This repeats the construction of graded Lagrangians by Seidel in [20], following the ideas of Kontsevich in [7].

Then we will apply the formalism in Section 4.1 to define Seidel gradings on two particular totally real tori in the $n$-fold symmetric product of a Riemann surface $\Sigma$. It is illustrated in [9] that by taking the Lagrangian Floer cohomology of these tori in the complement of a certain divisor $\nabla \subset \operatorname{Sym}^{n}(\Sigma)$, one obtains the fixed-point symplectic Khovanov homology group $K h_{\text {symp, inv }}(K)$. However, Manolescu also showed that these tori can be viewed as Heegaard tori $\mathbb{T}_{\widehat{\boldsymbol{\alpha}}}, \mathbb{T}_{\widehat{\boldsymbol{\beta}}}$ for the manifold $\Sigma(K) \#\left(S^{2} \times S^{1}\right)$. A holomorphic volume form on $W=\operatorname{Sym}^{n}(\Sigma) \backslash \nabla$ induces an absolute Maslov grading $\widetilde{R}$ on intersections of these tori when viewed inside $W$.

Furthermore, we have an identification of the set of Bigelow generators $\mathcal{G}$ with a generating set for the Heegaard Floer chain groups. This allows us to view $\widetilde{R}$ as a function on $\mathcal{G}$, and it in fact coincides with $P-Q+T$. However, when we view these tori inside all of $\operatorname{Sym}^{n}(\Sigma)$, this grading is no longer a priori consistent with Maslov index calculations (but rather also records intersections of 2 -gons with the factor $\nabla$ ).

We can use $R$ (a shifted version of $\widetilde{R}$ ) to define a filtration $\rho$ on $\widehat{C F}\left(\Sigma(K) \#\left(S^{2} \times S^{1}\right), \mathfrak{s}\right)$ for each torsion $\mathfrak{s} \in \operatorname{Spin}^{c}\left(\Sigma(K) \#\left(S^{2} \times S^{1}\right)\right)$. The definition for $\rho$ will appear to depend heavily on the braid $b$ chosen to represent the knot $K$. However, we will obtain an invariance result for this filtration in Section 5.2 in the form of Theorem 1.0.1.

4.1. Graded totally real submanifolds. First recall the following definition:

Definition 4.1.1. A real subspace $V \subset \mathbb{C}^{n}$ is called totally real (with respect to the standard complex structure if $\operatorname{dim}_{\mathbb{R}} V=n$ and $V \cap i V=0$. A half-dimensional submanifold $\mathcal{T}$ of an almost complex manifold $(Y, J)$ is called totally real if $T_{x} \mathcal{T} \cap J\left(T_{x} \mathcal{T}\right)=0$ for all $x \in \mathcal{T}$.

We will first work in the setting of a Kähler manifold $(Y, \Omega)$ such that $\Omega$ is exact and $c_{1}(Y)=0$. Recall that $Y$ then carries an almost-complex structure $J$ which is compatible with the symplectic form $\Omega$ - in this context, a totally real submanifold (with respect to $J$ ) is a generalization of a Lagrangian submanifold (with respect to $\Omega$ ). Let $\mathcal{T}$ and $\mathcal{T}^{\prime}$ be two totally real submanifolds of $Y$, intersecting transversely. 
Under these conditions, there is a well-defined abelian group $H F^{*}$ $\left(\mathcal{T}, \mathcal{T}^{\prime}\right)=H\left(C F^{*}\left(\mathcal{T}, \mathcal{T}^{\prime}\right), d\right)$ with a relative $\mathbb{Z}$-grading given by a Maslov index calculation. However, by a construction of Seidel in [20], this relative grading can be improved to an absolute $\mathbb{Z}$-grading.

Let $\mathfrak{T} \rightarrow Y$ be the natural fiber bundle whose fibers $\mathfrak{T}_{x}$ are the manifolds of totally real subspaces of $T_{x} Y$. Choosing a complex volume form $\Theta$ on $Y$ determines a square phase map $\theta: \mathfrak{T} \rightarrow \mathbb{C}^{*} / \mathbb{R}_{+} \cong S^{1}$ defined by $\theta(V)=$ $\Theta\left(e_{1} \wedge \cdots \wedge e_{n}\right)^{2}$, where $e_{1}, \ldots, e_{n}$ is any orthonormal basis for $V \subset T_{x} Y$.

Let $\widetilde{\mathfrak{T}} \rightarrow \mathfrak{T}$ be the infinite cyclic covering obtained by pulling back the covering $\mathbb{R} \rightarrow S^{1}$ via the map $\theta$. Consider the canonical section $s_{\mathcal{T}}: \mathcal{T} \rightarrow \mathfrak{T}$ given by $s_{\mathcal{T}}(x)=T_{x} \mathcal{T}$. This section induces a $S^{1}$-valued map $\theta_{\mathcal{T}}=\theta \circ s_{\mathcal{T}}$. In some cases, the section $s_{\mathcal{T}}$ can be lifted to a section $\widetilde{s}_{\mathcal{T}}: \mathcal{T} \rightarrow \widetilde{\mathfrak{T}}$ (inducing a lift $\widetilde{\theta}_{\mathcal{T}}: \mathcal{T} \rightarrow \mathbb{R}$ of the map $\left.\theta_{\mathcal{T}}\right)$. Let us assume such a lift exists.

Definition 4.1.2. A grading on $\mathcal{T}$ is a choice of lift $\widetilde{\theta}_{\mathcal{T}}: \mathcal{T} \rightarrow \mathbb{R}$.

Given such gradings on the submanifolds $\mathcal{T}$ and $\mathcal{T}^{\prime}$, one can define the absolute Maslov index $\mu(x) \in \mathbb{Z}$ for each element $x \in \mathcal{T} \cap \mathcal{T}^{\prime}[\mathbf{2 0}]$. This index is constructed using the Maslov index of paths in $\mathfrak{T}_{x}$, which is discussed in [17]. We will sometimes refer to the grading structure on $\mathcal{T}$ as $\widetilde{\mathcal{T}}$, and we will refer to the absolutely-graded Lagrangian Floer groups for the graded submanifolds $\widetilde{\mathcal{T}}$ and $\widetilde{\mathcal{T}}^{\prime}$ as $H F^{*}\left(\widetilde{\mathcal{T}}, \widetilde{\mathcal{T}}^{\prime}\right)$.

If $\Phi: Y \rightarrow Y$ is a symplectic automorphism with respect to the Kähler form $\Omega$, let $\Phi^{\mathfrak{T}}: \mathfrak{T} \rightarrow \mathfrak{T}$ denote the map given by $\Phi^{\mathfrak{T}}(V)=D \Phi(V)$. We recall the following definition:

Definition 4.1.3. Let $\Phi: Y \rightarrow Y$ be a symplectic automorphism, and suppose that there is a $\mathbb{Z}$-equivariant diffeomorphism $\widetilde{\Phi}: \widetilde{\mathfrak{T}} \mapsto \widetilde{\mathfrak{T}}$ which is a lift of $\Phi^{\mathfrak{T}}$. Then the pair $(\Phi, \widetilde{\Phi})$ is called a graded symplectic automorphism.

A graded symplectic automorphism $(\Phi, \widetilde{\Phi})$ acts on a graded Lagrangian submanifold $(L, \widetilde{L})$ by

$$
(\Phi, \widetilde{\Phi})(L, \widetilde{L})=\left(\Phi(L), \widetilde{\Phi} \circ \widetilde{L} \circ \Phi^{-1}\right) .
$$

Remark 4.1.4. We will often write $\widetilde{\Phi}$ to refer to the pair $(\Phi, \widetilde{\Phi})$ (and thus $\widetilde{\Phi}(\widetilde{L})$ will denote $(\Phi, \widetilde{\Phi})(L, \widetilde{L}))$.

As discussed in [20], many Lagrangian Floer identities can be extended to the absolutely-graded case. For instance, as absolutely-graded complexes, $C F\left(\widetilde{\mathcal{T}}, \widetilde{\mathcal{T}}^{\prime}\right) \cong\left(C F\left(\widetilde{\mathcal{T}}, \widetilde{\mathcal{T}}^{\prime}\right)\right)^{\vee}$, where " $\vee$ " denotes the dual complex.

Furthermore, if $\widetilde{\Phi}$ is a graded symplectic automorphism, then there is a natural isomorphism of absolutely-graded complexes $C F\left(\widetilde{\Phi}(\widetilde{\mathcal{T}}), \widetilde{\Phi}\left(\widetilde{\mathcal{T}}^{\prime}\right)\right) \cong$ $C F\left(\widetilde{\mathcal{T}}, \widetilde{\mathcal{T}}^{\prime}\right)$. 


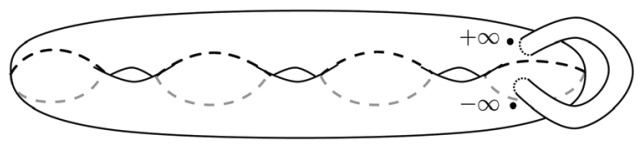

Figure 8. Stabilizing near $\pm \infty$.

4.2. From fork diagrams to Heegaard Floer homology. We summarize Manolescu's work in [9], describing a connection between Bigelow's fork diagram and a Heegaard diagram for the manifold $\Sigma(K) \#\left(S^{2} \times S^{1}\right)$.

We represent a knot $K$ as the plat closure of a braid $b \in B_{2 n}$, the braid group on $2 n$ strands, and obtain a fork diagram for $b$ by following the action the braid on the standard fork diagram, as described in Section 3.1.

Now let $P_{\mu} \in \mathbb{C}[t]$ be a polynomial with set of roots $\left\{\mu_{1}, \ldots \mu_{2 n}\right\}$, which is exactly the set of punctures in $\mathbb{C}$. We define an affine space $\widehat{S}=\{(u, z) \in$ $\left.\mathbb{C}^{2}: u^{2}+P_{\mu}(z)=0\right\} \subset \mathbb{C}^{2}$.

Also , for $k=1, \ldots, n$, define the subspaces $\widehat{\alpha}_{k}$ and $\widehat{\beta}_{k}$ of $\widehat{S}$ by

$$
\begin{aligned}
& \widehat{\alpha}_{k}=\left\{(u, z) \in \mathbb{C}: z=\alpha_{k}(t), \text { for some } t \in[0,1] ; u= \pm \sqrt{-P_{\mu}(z)}\right\} \text { and } \\
& \widehat{\beta}_{k}=\left\{(u, z) \in \mathbb{C}: z=\beta_{k}(t), \text { for some } t \in[0,1] ; u= \pm \sqrt{-P_{\mu}(z)}\right\}
\end{aligned}
$$

Note that the map $\widehat{S} \rightarrow \mathbb{C}$ defined by $(u, z) \mapsto z$ is a double branched covering with branch set equal to $\left\{\mu_{1}, \ldots, \mu_{2 n}\right\} \subset \mathbb{C}$. This means that $\widehat{S}$ can be seen as $\Sigma_{n-1}-\{ \pm \infty\}$, where $\Sigma_{n-1}$ is a Riemann surface of genus $(n-1)$. Furthermore, the $\widehat{\alpha}_{k}$ and $\widehat{\beta}_{k}$ are simple closes curves in $\widehat{S}$ which induce totally real tori $\mathbb{T}_{\widehat{\boldsymbol{\alpha}}}=\widehat{\alpha}_{1} \times \cdots \times \widehat{\alpha}_{n}, \mathbb{T}_{\widehat{\boldsymbol{\beta}}}=\widehat{\beta}_{1} \times \ldots \times \widehat{\beta}_{n} \subset \operatorname{Sym}^{n}(\widehat{S})$. We want a Heegaard diagram, so we stabilize this surface as shown in Figure 8 to acquire $\Sigma_{n}-\{ \pm \infty\}$.

Proposition 4.2.1 (Proposition 7.4 from [9]). The collection of data $\mathcal{H}=\left(\Sigma_{n} ; \widehat{\alpha}_{1}, \ldots, \widehat{\alpha}_{n} ; \widehat{\beta}_{1}, \ldots, \widehat{\beta}_{n} ;+\infty\right)$ is an admissible pointed Heegaard diagram for $\Sigma(K) \#\left(S^{2} \times S^{1}\right)$.

Now note that with respect to the covering map above, each puncture $\mu_{k} \in \mathbb{C}$ has a single point as its preimage. However, the preimage of a point $x \in \beta_{j} \cap \operatorname{int}\left(\alpha_{i}\right)$ consists of a pair of points upstairs. This gives a bijection between the intersection $\mathbb{T}_{\widehat{\boldsymbol{\alpha}}} \cap \mathbb{T}_{\widehat{\boldsymbol{\beta}}}$ and the set $\mathcal{G}$ of Bigelow generators as defined in Section 3.1. However, this identification isn't canonical, since for some $x \in \widetilde{\mathcal{Z}}-\tau$ it is only required that the pair $\left\{e_{x}, e_{x}^{\prime}\right\}$ is identified with the two preimages of $x$ upstairs. In any case, the grading function $R$ defined below will satisfy $R\left(e_{x}\right)=R\left(e_{x}^{\prime}\right)$. 
4.3. A grading induced by a volume form. Define a subset $W=$ $\operatorname{Sym}^{n}(\widehat{S})-\nabla$, where the anti-diagonal $\nabla$ is defined by

$$
\begin{aligned}
\nabla & =\left\{\left(u_{k}, z_{k}\right), k=1, \ldots, n: u_{k}^{2}+P_{\mu}\left(z_{k}\right)=0,\right. \\
\left(u_{i}, z_{i}\right) & \left.=\left(-u_{j}, z_{j}\right) \text { for some } i \neq j\right\}
\end{aligned}
$$

When we restrict to $W$, the Maslov grading on $\mathbb{T}_{\widehat{\boldsymbol{\alpha}}} \cap \mathbb{T}_{\widehat{\boldsymbol{\beta}}}$ can be lifted to an absolute Maslov $\mathbb{Z}$-grading by endowing the tori $\mathbb{T}_{\widehat{\boldsymbol{\alpha}}}, \mathbb{T}_{\widehat{\boldsymbol{\beta}}}$ with gradings in the sense of Section 4.1 via the choice of a particular holomorphic volume form.

Proposition 4.3.1 (Proposition 7.5 from [9]). There exists a complex volume form $\Theta$ on $W$ so that we can endow $\mathbb{T}_{\widehat{\boldsymbol{\alpha}}}$ and $\mathbb{T}_{\widehat{\boldsymbol{\beta}}}$ with gradings on the sense of Section 4.1. The resulting absolute Maslov grading (in $W$ ) on the elements of $\mathbb{T}_{\widehat{\boldsymbol{\alpha}}} \cap \mathbb{T}_{\widehat{\boldsymbol{\beta}}}$ is $P-Q+T$.

Proof. One can describe points in $\operatorname{Sym}^{n}(\widehat{S})$ by their coordinates $\left\{\left(u_{j}, z_{j}\right)\right\}_{j}$, where $u_{j}^{2}+P_{\mu}\left(z_{j}\right)=0$. Following Manolescu, we let the $\mathbb{C}$-valued $n$-form $\Theta$ on $\operatorname{Sym}^{n}(\widehat{S})$ be given by

$$
\Theta=\prod_{1 \leq i<j \leq n}\left(z_{i}-z_{j}\right) \cdot \prod_{k=1}^{n} \frac{d z_{k}}{u_{k}} .
$$

By using a basis of symmetric functions in the $z_{j}$ near any point on the diagonal $\Delta \subset \operatorname{Sym}^{n}(\widehat{S})$, Manolescu shows that $\Theta$ in fact gives a well-defined volume form on $W:=\operatorname{Sym}^{n}(\widehat{S}) \backslash \nabla$.

As described in Section 4.1, one can obtain from $\Theta$ two functions $\theta_{\alpha}$ : $\mathbb{T}_{\widehat{\boldsymbol{\alpha}}} \rightarrow S^{1}$ and $\theta_{\beta}: \mathbb{T}_{\widehat{\boldsymbol{\beta}}} \rightarrow S^{1}$. A point $\mathbf{x} \in \mathbb{T}_{\widehat{\boldsymbol{\beta}}}$ has coordinates $\left\{\left(u_{j}, z_{j}\right)\right\}_{j}$ where $z_{j}=\beta_{j}\left(t_{j}\right)$ for some $t_{j} \in[0,1]$, and $u_{j}= \pm \sqrt{-P_{\mu}\left(\beta_{j}\left(t_{j}\right)\right)}$. So, we can write

$$
\theta_{\beta}(\mathbf{x})=\prod_{1 \leq i<j \leq n}\left(\beta_{i}\left(t_{i}\right)-\beta_{j}\left(t_{j}\right)\right)^{2} \cdot \prod_{k=1}^{n} \frac{\beta_{j}^{\prime}\left(t_{j}\right)^{2}}{-P_{\mu}\left(\beta_{j}\left(t_{j}\right)\right)},
$$

and write $\theta_{\alpha}(\mathbf{x})$ similarly for $\mathbf{x} \in \mathbb{T}_{\widehat{\boldsymbol{\alpha}}}$.

A choice of $\mathbb{R}$-valued lifts $\widetilde{\theta}_{\alpha}$ and $\widetilde{\theta}_{\beta}$ of $\theta_{\alpha} \theta_{\beta}$ will induce an absolute Maslov grading on $\mathbb{T}_{\widehat{\boldsymbol{\alpha}}} \cap \mathbb{T}_{\widehat{\boldsymbol{\beta}}} \subset W$. Note that $\theta_{\alpha}$ has a constant value of $1 \in S^{1}$; it is shown in $[\mathbf{9}]$ (by examination of the function $\theta_{\beta}$ ) that any choice of lifts $\widetilde{\theta}_{\alpha}, \widetilde{\theta}_{\beta}$ will induce a Maslov grading, which agrees with $P-Q+T$ upto an overall shift, and the same argument applies here.

In [9], the absolute Maslov grading is fixed to be exactly $P-Q+T$ by choosing $\widetilde{\theta}_{\beta}$ to be obtained continuously from $\widetilde{\theta}_{\alpha}$ by following the family of crossingless matchings (induced by the braid action) starting at $\left\{\alpha_{1}, \ldots, \alpha_{n}\right\}$ and ending at $\left\{\beta_{1}, \ldots, \beta_{n}\right\}$; this effectively sets $\widetilde{R}\left(\mathbf{x}_{0}\right)=0$ for a distinguished 
Table 2. Distributions of $\widetilde{R}$ and $R$ for $\sigma_{2}^{3} \in B_{4}$.

\begin{tabular}{|c|l|}
\hline$\widetilde{R}$ & $\mathcal{G}$ elements \\
\hline \hline 0 & $x_{1} x_{4}, x_{1} u, x_{1} u^{\prime}, t x_{4}, t^{\prime} x_{4}$ \\
\hline 1 & $\begin{array}{l}x_{2} v, x_{2} v^{\prime}, s x_{3}, s^{\prime} x_{3}, s v, s^{\prime} v, \\
s v^{\prime}, s^{\prime} v^{\prime}\end{array}$ \\
\hline 2 & $t u, t^{\prime} u, t u^{\prime}, t^{\prime} u^{\prime}$ \\
\hline 3 & $x_{2} x_{3}$ \\
\hline
\end{tabular}

\begin{tabular}{|c|l|}
\hline$R$ & $\mathcal{G}$ elements \\
\hline \hline $1 / 2$ & $x_{1} x_{4}, x_{1} u, x_{1} u^{\prime}, t x_{4}, t^{\prime} x_{4}$ \\
\hline $3 / 2$ & $\begin{array}{l}x_{2} v, x_{2} v^{\prime}, s x_{3}, s^{\prime} x_{3}, s v, s^{\prime} v, \\
s v^{\prime}, s^{\prime} v^{\prime}\end{array}$ \\
\hline $5 / 2$ & $t u, t^{\prime} u, t u^{\prime}, t^{\prime} u^{\prime}$ \\
\hline $7 / 2$ & $x_{2} x_{3}$ \\
\hline
\end{tabular}

generator $\mathbf{x}_{0} \in \mathbb{T}_{\widehat{\boldsymbol{\alpha}}} \cap \mathbb{T}_{\widehat{\boldsymbol{\beta}}}$. In our case, there is no such distinguished generator. Instead, we set $\widetilde{\theta}_{\alpha} \equiv 0$ and then choose the lift $\widetilde{\theta}_{\beta}$ in a way that the induced Maslov grading $\widetilde{R}$ satisfies $\widetilde{R}(\mathbf{x})=(P-Q+T)(\mathbf{x})$ for some choice of generator $\mathbf{x} \in \mathbb{T}_{\widehat{\boldsymbol{\alpha}}} \cap \mathbb{T}_{\widehat{\boldsymbol{\beta}}}$. Necessarily we will then have that $\widetilde{R}=P-Q+T$.

We can now view $\widetilde{R}$ as a function both on $\mathbb{T}_{\widehat{\boldsymbol{\alpha}}} \cap \mathbb{T}_{\widehat{\boldsymbol{\beta}}}$ and on the set $\mathcal{G}$ of Bigelow generators. Now we define a rational number $s_{R}(b, D)$, which will depends on properties of the braid $b$ and of the oriented link diagram $D$ which is its plat closure. Denote by $\epsilon(b)$ the sum of the powers (with sign) of the braid group generators making up the word $b$, and denote by $w$ the writhe of the diagram $D$. Then let

$$
s_{R}(b, D)=\frac{\epsilon(b)-w(D)-2 n}{4} \in \mathbb{Q} .
$$

Then for $\mathbf{e} \in \mathcal{G}$, define $R(\mathbf{e})=\widetilde{R}(\mathbf{e})+s_{R}=P(\mathbf{e})-Q(\mathbf{e})+T(\mathbf{e})+s_{R}$.

One should note that for any $x \in \widetilde{\mathcal{Z}} \backslash \tau$, we have that $Q^{*}\left(e_{x}^{\prime}\right)=Q^{*}\left(e_{x}\right)+1$ and $P^{*}\left(e_{x}^{\prime}\right)=P^{*}\left(e_{x}\right)+1$. As a result, $R\left(e_{x}^{\prime} \mathbf{y}\right)=R\left(e_{x} \mathbf{y}\right)$; following [9], we say that the grading $R$ is stable.

4.4. Computing $\mathbf{R}$ for the left-handed trefoil. Here we have that $n=$ $2, \epsilon=3$, and $w=-3$, and so

$$
s_{R}(b, D)=\frac{(3)-(-3)-2(2)}{4}=\frac{1}{2} .
$$

Combining this with Table 1, one obtains the distributions of $\widetilde{R}$ and $R$ seen in Table 2 .

4.4.1. Drawing Heegaard diagrams. Given a fork diagram, it is straightforward (albeit sometimes tedious) to construct the admissible pointed Heegaard diagram discussed in Proposition 4.2.1. Figure 9(a) shows a standard fork diagram with six punctures (where the handle arcs are omitted). Cutting along the dashed arcs produces three disks, each with two punctures. The double cover of each such disk branched over the punctures is an annulus, as shown in Figure 9(b). One can reglue the annuli to form a genus-two 
(a)
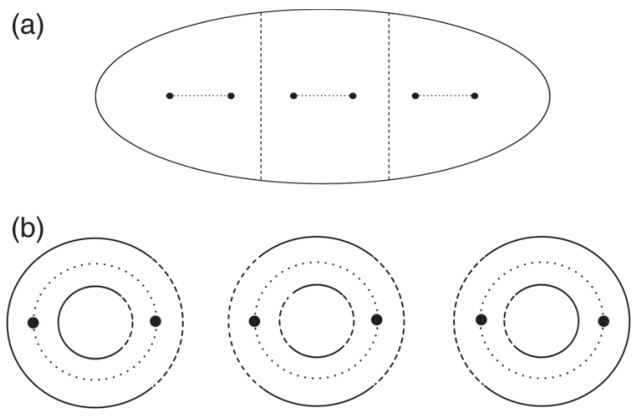

(c)

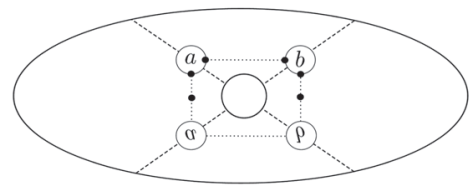

Figure 9. Producing a pointed Heegaard diagram from a fork diagram. The dotted arcs (resp. circles) represent the $\alpha_{i}$ (resp. their covers $\widehat{\alpha}_{i}$ ) and black dots represent punctures. (a) A fork diagram cut along dashed arcs, (b) The annuli covering the three pieces from Figures 9(a) and (c) The gluedup genus-two surface.

surface with two boundary components, as shown in Figure 9(c). Capping off the boundary components and stabilizing the surface with a handle whose feet lie near $\pm \infty$ yields the required pointed Heegaard diagram.

During the invariance proof, we will exhibit local pictures of Heegaard diagrams covering local pictures of fork diagrams with three punctures. In this case, one should cut the fork diagram into two disks (one with two punctures and one with one), as in Figure 10(a). The branched covers of these pieces are an annulus and a disk, respectively; gluing yields a genusone surface with one boundary component, as seen in Figure 10(c).

4.5. Intersections with the anti-diagonal. However, as observed in [22], the volume form $\Theta$ has an order-one zero along the antidiagonal $\nabla$. Therefore, $R$ is not compatible with Maslov index counts in all of $\operatorname{Sym}^{n}(\widehat{S})$.

Let $\phi \in \pi_{2}(\mathbf{x}, \mathbf{y})$ be counted by a term in $\widehat{\partial}(\mathbf{x})$. If $\phi$ intersects $\nabla$ with multiplicity $k \in \mathbb{Z}$ (it can be arranged that $k \geq 0$, with equality only if $\phi$ completely avoids $\nabla)$, then $[\mathbf{2 2}]$ gives that $R(\mathbf{x})-R(\mathbf{y})=2 k+1$.

More generally, one can say that if $\phi \in \pi_{2}(\mathbf{x}, \mathbf{y})$ with $n_{+\infty}(\phi)=0$, then

$$
R(\mathbf{x})-R(\mathbf{y})=\mu(\phi)+2([\phi] \cdot[\nabla])=\operatorname{gr}(\mathbf{x}, \mathbf{y})+2([\phi] \cdot[\nabla]) .
$$

Now for each torsion $\mathfrak{s} \in \operatorname{Spin}^{c}\left(\Sigma(K) \#\left(S^{2} \times S^{1}\right)\right)$ define $\rho: \mathfrak{U}_{\mathfrak{s}} \rightarrow \mathbb{Q}$ by $\rho(\mathbf{x})=R(\mathbf{x})-\tilde{g r}(\mathbf{x})$. Then we have that if $\mathbf{x}, \mathbf{y} \in \mathfrak{U}_{\mathfrak{s}}$ for $\mathfrak{s}$ torsion and $\phi \in \pi_{2}(\mathbf{x}, \mathbf{y})$ with $n_{+\infty}(\phi)=0, \rho(\mathbf{x})-\rho(\mathbf{y})=2[\phi] \cdot[\nabla]$. 

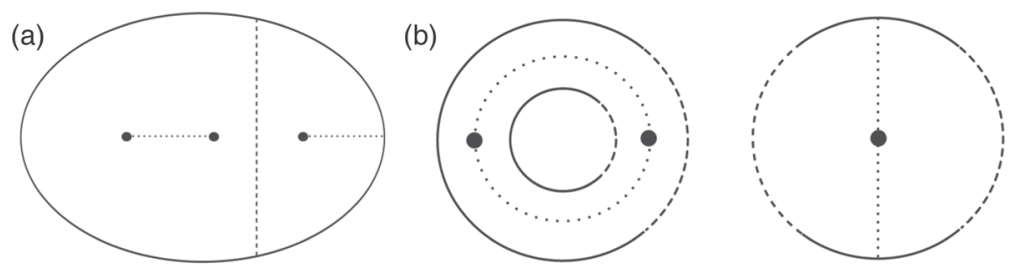

(c)

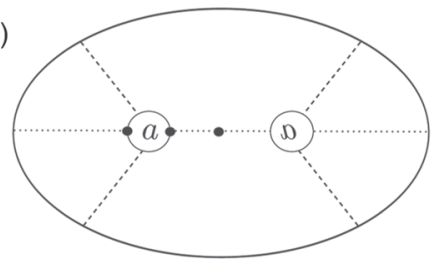

Figure 10. A local region of a pointed Heegaard diagram covering a thrice-punctured region of a fork diagram. (a) A region of a fork diagram cut along dashed arcs, (b) The annulus and disk covering the two pieces from Figures 10(a) and (c) The glued-up genus-one surface.

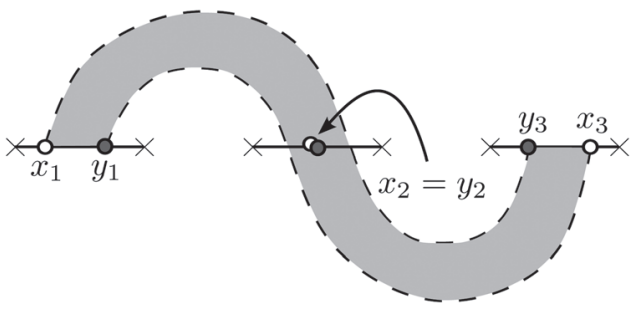

Figure 11. A portion of a fork diagram. The domain $\pi(\mathcal{D}(\phi))$ is shaded, the $\boldsymbol{\alpha}$ arcs are solid, and the $\boldsymbol{\beta}$ arcs are dashed.

Now $\rho$ provides a filtration grading on the factor $\widehat{C F}(\mathcal{H}, \mathfrak{s})$ for each torsion $\mathfrak{s}$.

4.5.1. A schematic example of non-trivial intersection. For the sake of concreteness, let us see an example of a 2-gon whose intersection number with the anti-diagonal is nonzero. Figure 11 shows a portion of a fork diagram induced by some braid in $B_{6}$. Let $\mathbf{x}, \mathbf{y} \in \mathcal{G}$ be the Bigelow generators whose components are indicated in Figure 11.

Figure 12 shows the Heegaard diagram of genus 3 obtained from the fork diagram via Theorem 4.2.1, and let $\pi$ denote the branched covering map. Let $\widehat{\mathbf{x}}, \widehat{\mathbf{y}} \in \mathbb{T}_{\widehat{\boldsymbol{\alpha}}} \cap \mathbb{T}_{\widehat{\boldsymbol{\beta}}}$ have components as indicated in the Heegaard diagram, where $\pi\left(\widehat{x_{i}}\right)=x_{i}$ and $\pi\left(\widehat{y}_{i}\right)=y_{i}$ for $i=1,2,3$. The shaded region in 


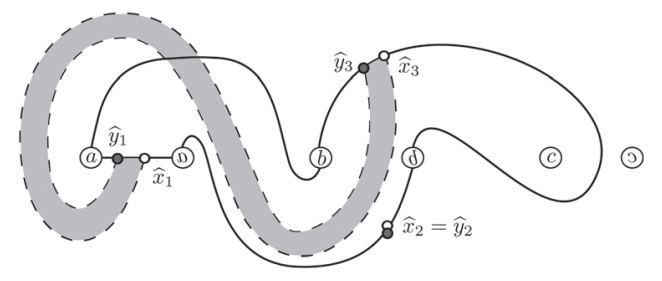

Figure 12. A Heegaard diagram induced by Figure 11. The domain $\mathcal{D}(\phi)$ is shaded.

Figure 12 is the domain $\mathcal{D}(\phi)$ of a 2 -gon $\phi \in \pi_{2}(\widehat{\mathbf{x}}, \widehat{\mathbf{y}})$ and the shaded region in Figure 11 is its image $\pi(\mathcal{D}(\phi))$.

Note that for each $i, \pi^{-1}\left(x_{i}\right)$ contains two points; the point $\widehat{x}_{2}$ and $\widehat{y}_{2}$ are both chosen to be the preimage of $x_{2}=y_{2}$ which lies outside of the domain $\mathcal{D}(\phi)$.

One can see that $\widetilde{g r}(\widehat{\mathbf{x}})-\widetilde{g r}(\widehat{\mathbf{y}})=\mu(\phi)=1$. However, $[\phi] \cdot[\nabla]=1$, and one can verify from the fork diagram that indeed $R(\mathbf{x})-R(\mathbf{y})=3$.

4.5.2. The anti-diagonal and Heegaard multi-diagrams. Throughout the rest of Section 4, we will assume that $\Sigma$ is a genus- $n$ Heegaard surface arising as the double branched cover of $S^{2}$, as described in the discussion preceding Proposition 4.2.1, with basepoint $+\infty \in \Sigma$. Further, assume that $\boldsymbol{\alpha}, \boldsymbol{\beta}$, and $\boldsymbol{\beta}^{\prime}$ be $n$-tuples of attaching curves on $\Sigma$ such that $\boldsymbol{\beta}^{\prime}$ differs from $\boldsymbol{\beta}$ by a pointed handeslide or pointed isotopy.

We will find in Section 5 that Birman moves will induce sequences of Heegaard moves such that only the initial and final $\alpha$ and $\beta$ circles are lifts of arcs from fork diagrams. However, one should consider $\nabla \subset \operatorname{Sym}^{n}(\Sigma)$ as being determined by the branched covering map $\pi: \Sigma \rightarrow \mathbb{C}$ (and thus being a well-defined feature of intermediate Heegaard diagrams).

We will analyze several types of 3-gons in the invariance proof in Section 5. For $\mathbf{x} \in \mathbb{T}_{\boldsymbol{\alpha}} \cap \mathbb{T}_{\boldsymbol{\beta}}$ and $\mathbf{y} \in \mathbb{T}_{\boldsymbol{\alpha}} \cap \mathbb{T}_{\boldsymbol{\beta}^{\prime}}$, let $\psi \in \pi_{2}\left(\mathbf{x}, \boldsymbol{\theta}_{\boldsymbol{\beta}, \boldsymbol{\beta}^{\prime}}, \mathbf{y}\right)$ be a 3 -gon class avoiding the basepoint with $\mu(\psi)=0$, where the domain $\mathcal{D}(\psi)$ has one of the two types discussed in Section 2.1.1. If $\mathcal{D}(\psi)$ is of the first type (a sum of $n$ disjoint 3 -sided regions $\left.\mathcal{D}_{1}, \ldots, \mathcal{D}_{n}\right)$, a point in $\operatorname{Im}(\psi) \subset \operatorname{Sym}^{n}(\Sigma)$ is of the form $\mathbf{x}=\left\{x_{1}, \ldots, x_{n}\right\}$, where each $x_{i} \in \mathcal{D}_{i}$. Further assume that at least $n-1$ of the regions $\mathcal{D}_{i}$ are small 3 -sided regions of the type appearing in Figure 13. It can easily be arranged that

$$
\mathcal{D}_{i} \cap \pi^{-1}\left(\pi\left(\mathcal{D}_{j}\right)\right)=\emptyset \quad \text { for } \quad i \neq j
$$

and so $\mathbf{x} \notin \nabla$.

However, in Section 5.3.4, we will encounter a case in which $\mathcal{D}(\psi)$ is of the second type (a sum of $(n-1)$ disjoint regions $\mathcal{D}_{1}, \ldots, \mathcal{D}_{(n-1)}$, where the first $(n-2)$ are 3 -sided regions and the last is a 6 -sided region with one obtuse angle). Additionally, assume that $\mathcal{D}_{1}, \ldots, \mathcal{D}_{(n-2)}$ are as shown in Figure 13. 


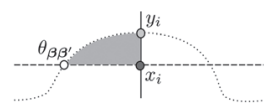

Figure 13. A small 3-sided region appearing in a small isotopy.

In this case, a point in $\operatorname{Im}(\psi) \subset \operatorname{Sym}^{n}(\Sigma)$ is of the form $\mathbf{x}=\left\{x_{1}, \ldots, x_{n}\right\}$, where $x_{i} \in \mathcal{D}_{i}$ for $1 \leq i \leq n-2$ and $x_{(n-1)}, x_{n} \in \mathcal{D}_{(n-1)}$. An analog of equation (4.1) can also be achieved here; as a result, $\mathbf{x} \notin \nabla$ as long as it is not the case that $x_{g} \neq x_{(n-1)}$ and $\pi\left(x_{n}\right)=\pi\left(x_{(n-1)}\right)$. We will show that this is impossible by arranging that $\pi^{-1}\left(\pi\left(\mathcal{D}_{(n-1)}\right)\right)$ has two connected components (one of which is $\mathcal{D}_{(n-1)}$ itself).

4.5.3. The anti-diagonal and periodic domains. Let $\alpha_{i} \cap \beta_{i}=\left\{x_{i}, y_{i}\right\}$, as labelled in Figure 2, and let $\mathbf{x}=\left\{x_{1}, \ldots, x_{n}\right\}$ and $\mathbf{y}=\left\{y_{1}, \ldots, y_{n}\right\}$. Assume without loss of generality that such a handleslide is of $\alpha_{1}$ over $\alpha_{2}$. Then let the domains $\mathcal{D}_{i}^{\boldsymbol{\alpha} \boldsymbol{\beta}}$ be the ones defined in Section 2.2. In this context, we have the following fact:

Lemma 4.5.1. Let $(\Sigma ; \boldsymbol{\alpha} ; \boldsymbol{\beta} ;+\infty)$ be the pointed Heegaard diagram mentioned above. Let $\phi \in \pi_{2}(\mathbf{v}, \mathbf{v})$, where $\mathbf{v}=\mathbf{x}$ or $\mathbf{v}=\mathbf{y}$. Then $\phi$ avoids the anti-diagonal $\nabla$.

Proof. Without loss of generality, let $\mathbf{v}=\mathbf{x}$. Letting $\mathcal{D}_{i}^{\boldsymbol{\alpha} \boldsymbol{\beta}}$ be as in Section 2.2 , one can see that for each $i$, there is a class $\phi_{i} \in \pi_{2}(\mathbf{x}, \mathbf{x})$ with $\mathcal{D}\left(\phi_{i}\right)=\mathcal{D}_{i}^{\boldsymbol{\alpha} \boldsymbol{\beta}}$.

First assume that $\boldsymbol{\beta}$ differs from $\boldsymbol{\alpha}$ by a pointed isotopy. Then every point in $\operatorname{Im}\left(\phi_{1}\right) \subset \operatorname{Sym}^{n}(\Sigma)$ is of the form $\left\{x, x_{2}, \ldots, x_{n}\right\}$, where $x \in \mathcal{D}_{1}^{\alpha}$. It can be arranged that $\mathcal{D}_{1}^{\boldsymbol{\alpha} \boldsymbol{\beta}}$ avoids the branched covering pre-image "twin" of $x_{j}$ for each $j \geq 2$, and so $\phi_{1}$ avoids $\nabla$.

Instead assume that $\boldsymbol{\beta}$ differs from $\boldsymbol{\alpha}$ by a pointed handleslide of $\alpha_{1}$ over $\alpha_{2}$, as in Figure 2. The argument for the isotopy case above implies that $\phi_{i}$ avoids $\nabla$ for $i \geq 2$. Note that we can write $\phi_{1}=\phi_{1,1}+\phi_{1,2}$, where $\phi_{1,1} \in$ $\pi_{2}(\mathbf{x}, \mathbf{y})$ has domain given by the annular component of $\mathcal{D}_{1}^{\boldsymbol{\alpha} \boldsymbol{\beta}}$ (with local coefficient +1$)$ and $\phi_{1,2}, \in \pi_{2}(\mathbf{y}, \mathbf{x})$ has domain given by the small two-sided component of $\mathcal{D}_{1}^{\boldsymbol{\alpha} \boldsymbol{\beta}}$ (with local coefficient -1 ). By an argument analogous to that in the isotopy case, $\phi_{1,2}$ avoids $\nabla$. Note that any point in $\operatorname{Im}\left(\phi_{1,1}\right)$ is of the form $\left\{x, x_{2}, \ldots, x_{n}\right\}$, where $x$ lies in the annular component. It can be arranged that this annular component avoids the branched covering pre-image "twin" of $x_{j}$ for each $j \geq 2$, and so $\phi_{1,1}$ avoids $\nabla$ as well.

In either case, $\phi$ can be written as a sum (concatenation) of the classes $\phi_{i}$, and so it can be arranged that $\phi$ avoids $\nabla$. 


\subsubsection{The anti-diagonal and the homotopy for a small pointed isotopy.}

\section{Invariance of the filtration}

Here we will prove a few facts that we will use in our invariance proofs.

Remark 5.0.2. From now on, we will suppress the hat when discussing a lift $\widehat{\alpha}$ of an arc $\alpha$ unless the distinction is not obvious from the context.

Now let $\left(\Sigma ; \boldsymbol{\alpha}^{\prime} ; \boldsymbol{\alpha} ; \boldsymbol{\beta} ; z\right)$ be a pointed Heegaard triple-diagram, where the set of attaching circles $\boldsymbol{\alpha}^{\prime}$ is obtained from $\boldsymbol{\alpha}$ a pointed handleslide or pointed isotopy. Then $(\Sigma ; \boldsymbol{\alpha} ; \boldsymbol{\beta} ; z)$ and $\left(\Sigma ; \boldsymbol{\alpha}^{\prime} ; \boldsymbol{\beta} ; z\right)$ are two pointed Heegaard diagrams for the same 3 -manifold $M$. Recall that in fact $\left(\Sigma ; \boldsymbol{\alpha}^{\prime} ; \boldsymbol{\alpha} ; z\right)$ is an admissible pointed Heegaard diagram for $\#_{n}\left(S^{1} \times S^{2}\right)$. There is a natural choice of top-degree generator $\boldsymbol{\theta}_{\boldsymbol{\alpha}^{\prime} \boldsymbol{\alpha}} \in \mathbb{T}_{\boldsymbol{\alpha}^{\prime}} \cap \mathbb{T}_{\boldsymbol{\alpha}}$. We also assume that $\mathcal{H}_{\boldsymbol{\alpha} \boldsymbol{\beta}}:=(\Sigma ; \boldsymbol{\alpha} ; \boldsymbol{\beta} ; z)$ and $\mathcal{H}_{\boldsymbol{\alpha}^{\prime} \boldsymbol{\beta}}:=\left(\Sigma ; \boldsymbol{\alpha}^{\prime} ; \boldsymbol{\beta} ; z\right)$ are admissible - Proposition 2.2.4 thus implies that the triple-diagram is admissible also. Recall that there is a 3 -gon counting chain homotopy equivalence

$$
\widehat{f}_{\boldsymbol{\alpha}^{\prime}, \boldsymbol{\alpha}, \boldsymbol{\beta}}\left(\boldsymbol{\theta}_{\boldsymbol{\alpha}^{\prime} \boldsymbol{\alpha}} \otimes \cdot\right): \widehat{C F}\left(\mathcal{H}_{\boldsymbol{\alpha} \boldsymbol{\alpha}}\right) \rightarrow \widehat{C F}\left(\mathcal{H}_{\boldsymbol{\alpha}^{\prime} \boldsymbol{\alpha}}\right) \text {. }
$$

Now for any $\mathbf{x} \in \mathbb{T}_{\boldsymbol{\alpha}^{\prime}} \cap \mathbb{T}_{\boldsymbol{\alpha}}, \mathbf{y} \in \mathbb{T}_{\boldsymbol{\alpha}} \cap \mathbb{T}_{\boldsymbol{\beta}}$, and $\mathbf{z} \in \mathbb{T}_{\boldsymbol{\alpha}^{\prime}} \cap \mathbb{T}_{\boldsymbol{\beta}}$, there is a well-defined map

$$
\mathfrak{s}_{z}: \pi_{2}(\mathbf{x}, \mathbf{y}, \mathbf{z}) \rightarrow \operatorname{Spin}^{c}(X),
$$

where $X$ is the cobordism induced by the Heegaard move. Since $X$ is induced by a handleslide or any isotopy, the cobordism is in fact a cylinder. Therefore, if $\boldsymbol{\theta}_{\boldsymbol{\alpha}^{\prime} \boldsymbol{\alpha}} \in \mathbb{T}_{\boldsymbol{\alpha}^{\prime}} \cap \mathbb{T}_{\boldsymbol{\alpha}}$ represents the top-degree generator of $\widehat{C F}\left(\#^{n}\left(S^{2} \times S^{1}\right)\right)$, then for some $\psi \in \pi_{2}\left(\boldsymbol{\theta}_{\boldsymbol{\alpha}^{\prime} \boldsymbol{\alpha}}, \mathbf{y}, \mathbf{z}\right), \mathfrak{s}_{z}(\psi)$ is completely determined by either restriction $\mathfrak{s}_{z}(\mathbf{y})$ or $\mathfrak{s}_{z}(\mathbf{z})$.

We will need the following fact about the absolute grading $\widetilde{g r}$ :

Lemma 5.0.3. Let $\left(\Sigma ; \boldsymbol{\alpha}^{\prime} ; \boldsymbol{\alpha} ; \boldsymbol{\beta} ; z\right)$ be an admissible pointed Heegaard triplediagram such that $\boldsymbol{\alpha}^{\prime}$ differs from $\boldsymbol{\alpha}$ by a pointed isotopy or pointed handleslide. Then if $\mathbf{x} \in \mathfrak{U}_{\mathfrak{s}} \subset \mathbb{T}_{\boldsymbol{\alpha}} \cap \mathbb{T}_{\boldsymbol{\beta}}$ for $\mathfrak{s} \in \operatorname{Spin}^{c}\left(Y_{\boldsymbol{\alpha} \boldsymbol{\beta}}\right)$ torsion and $\mathbf{y} \in \mathbb{T}_{\boldsymbol{\alpha}^{\prime}} \cap \mathbb{T}_{\boldsymbol{\beta}}$ is a generator appearing with nonzero coefficient in the expansion of $\widehat{f}_{\boldsymbol{\alpha}^{\prime}, \boldsymbol{\alpha}, \boldsymbol{\beta}}\left(\boldsymbol{\theta}_{\boldsymbol{\alpha}^{\prime} \boldsymbol{\alpha}} \otimes \mathbf{x}\right), \tilde{g r}(\mathbf{y})=\tilde{g r}(\mathbf{x})$.

Proof. Let $X$ be the cobordism induced by the Heegaard move and choose some $\mathfrak{t} \in \operatorname{Spin}^{c}(X)$ restricting to $\mathfrak{s}$ on $Y_{\boldsymbol{\alpha} \boldsymbol{\beta}}$ and $\mathfrak{s}^{\prime}$ on $Y_{\boldsymbol{\alpha}^{\prime} \boldsymbol{\beta}}$, where $\mathbf{y} \in \mathfrak{U}_{\mathfrak{s}^{\prime}} \subset$ $\mathbb{T}_{\boldsymbol{\alpha}^{\prime}} \cap \mathbb{T}_{\boldsymbol{\beta}}$. The absolute grading $\widetilde{g r}$ is uniquely characterized in $[\mathbf{1 1}]$ by several properties, one of which implies that

$$
\widetilde{g r}(\mathbf{y})-\widetilde{g r}(\mathbf{x})=\frac{c_{1}^{2}(\mathfrak{t})-2 \xi(X)-3 \sigma(X)}{4} .
$$

Since $X$ is in fact a cylinder, the right-hand side vanishes. 
Letting $\widetilde{\boldsymbol{\alpha}}$ differ from $\boldsymbol{\alpha}$ by a small pointed isotopy and working in the pointed Heegaard quadruple-diagram $\left(\Sigma ; \widetilde{\boldsymbol{\alpha}} ; \boldsymbol{\alpha}^{\prime} ; \boldsymbol{\alpha} ; \boldsymbol{\beta} ; z\right)$, one can make an analogous observation regarding $\operatorname{Spin}^{c}$ structures associated to 4-gons.

Recall that the filtration $\rho$ is only well-defined on the summands $\widehat{C F}\left(\Sigma(K) \#\left(S^{2} \times S^{1}\right), \mathfrak{s}\right)$ with $\mathfrak{s} \in \operatorname{Spin}^{c}\left(\Sigma(K) \#\left(S^{2} \times S^{1}\right)\right)$ torsion. However, due to the observations above, everything in sight will be $\mathrm{Spin}^{c}$-equivalent and we will suppress $\operatorname{Spin}^{c}$ structures in notation when proving many of the lemmas in this section.

Below we discuss Heegaard diagrams obtained from braids. The Birman stabilization move on braids induces in the Heegaard diagram a Heegaard stabilization followed by two handleslides. For a Heegaard diagram $\mathcal{H}$, stabilization amounts to taking a connected sum with $\mathcal{H}_{0}$, the standard genus-one pointed Heegaard diagram for $S^{3}$ with $\boldsymbol{\alpha} \cap \boldsymbol{\beta}=\{x\}$, where the connected sum is performed near the respective basepoints of the diagrams. Ozsváth and Szabó showed in $[\mathbf{1 3}]$ that as chain complexes, $\widehat{C F}(\mathcal{H}) \cong \widehat{C F}\left(\mathcal{H} \# \mathcal{H}_{0}\right)$. If $\mathcal{H}$ is a Heegaard diagram for $\Sigma(K) \#\left(S^{2} \times S^{1}\right)$ obtained from a braid $b$, we extend $R$ and $\rho$ to $\widehat{C F}\left(\mathcal{H} \# \mathcal{H}_{0}, \mathfrak{s} \# \mathfrak{s}_{0}\right)$ for each torsion $\mathfrak{s}$ by setting $R(x \mathbf{y})=R(\mathbf{y})$.

Let us first establish some terminology that will be used in Lemma 5.0.6 to follow.

Definition 5.0.4. Let $(\Sigma ; \boldsymbol{\alpha} ; \boldsymbol{\beta} ; z)$ and $\left(\Sigma ; \boldsymbol{\alpha}^{\prime} ; \boldsymbol{\beta}^{\prime} ; z\right)$ be two admissible Heegaard diagrams of genus $n$ appearing in some sequence of Heegaard moves connecting two diagrams covering fork diagrams, and let $\nabla \subset \operatorname{Sym}^{n}(\Sigma)$ denote the anti-diagonal.

(i) If $\boldsymbol{\beta}^{\prime}=\boldsymbol{\beta}$ and $\boldsymbol{\alpha}^{\prime}$ differs from $\boldsymbol{\alpha}$ by a pointed isotopy or pointed handleslide, then a $\boldsymbol{\alpha}$-triangle injection is a function $g: \mathbb{T}_{\boldsymbol{\alpha}} \cap \mathbb{T}_{\boldsymbol{\beta}} \hookrightarrow$ $\mathbb{T}_{\boldsymbol{\alpha}^{\prime}} \cap \mathbb{T}_{\boldsymbol{\beta}}$ such that the following hold:

(a) There is a Heegaard triple-diagram $\left(\Sigma ; \boldsymbol{\alpha}^{+} ; \boldsymbol{\alpha} ; \boldsymbol{\beta} ; z\right)$ (where for each $k, \alpha_{k}^{+}$is isotopic to $\alpha_{k}^{\prime}$ and intersects $\alpha_{k}$ transversely in two points) such that for each $\mathbf{x} \in \mathbb{T}_{\boldsymbol{\alpha}} \cap \mathbb{T}_{\boldsymbol{\beta}}$, there is a 3-gon class $\psi_{g}^{+} \in$ $\pi_{2}\left(\boldsymbol{\theta}_{\boldsymbol{\alpha}^{+} \boldsymbol{\alpha}}, \mathbf{x}, \mathbf{y}^{+}\right)$with $\mu\left(\psi_{g}^{+}\right)=0, \psi_{g}^{+} \cap \nabla=\emptyset$, and $n_{z}\left(\psi_{g}^{+}\right)=0$, where $\mathbf{y}^{+} \in \mathbb{T}_{\boldsymbol{\alpha}} \cap \mathbb{T}_{\boldsymbol{\alpha}^{+}}$is the nearest neighbor to $g(\mathbf{x})$.

(b) There is a Heegaard triple-diagram $\left(\Sigma ; \boldsymbol{\alpha} ; \boldsymbol{\alpha}^{-} ; \boldsymbol{\beta} ; z\right)$ (where for each $k, \alpha_{k}^{-}$is isotopic to $\alpha_{k}^{\prime}$ and intersects $\alpha_{k}$ transversely in two points) such that for each $\mathbf{x} \in \mathbb{T}_{\boldsymbol{\alpha}}^{\prime} \cap \mathbb{T}_{\boldsymbol{\beta}}$, there is a 3-gon class $\psi_{g}^{-} \in \pi_{2}\left(\boldsymbol{\theta}_{\boldsymbol{\alpha} \boldsymbol{\alpha}^{-}}, \mathbf{y}^{-}, \mathbf{x}\right)$ with $\mu\left(\psi_{g}^{-}\right)=0, \psi_{g}^{-} \cap \nabla=\emptyset$, and $n_{z}\left(\psi_{g}^{-}\right)=0$, where $\mathbf{y}^{-} \in \mathbb{T}_{\boldsymbol{\alpha}} \cap \mathbb{T}_{\boldsymbol{\alpha}^{-}}$is the nearest neighbor to $g(\mathbf{x})$.

(ii) If $\boldsymbol{\alpha}^{\prime}=\boldsymbol{\alpha}$ and $\boldsymbol{\beta}^{\prime}$ differs from $\boldsymbol{\beta}$ by a pointed isotopy or pointed handleslide, then a $\boldsymbol{\beta}$-triangle injection is a function $g: \mathbb{T}_{\boldsymbol{\alpha}} \cap \mathbb{T}_{\boldsymbol{\beta}} \hookrightarrow \mathbb{T}_{\boldsymbol{\alpha}} \cap \mathbb{T}_{\boldsymbol{\beta}^{\prime}}$ such that the following hold: 
(a) There is a Heegaard triple-diagram $\left(\Sigma ; \boldsymbol{\alpha} ; \boldsymbol{\beta} ; \boldsymbol{\beta}^{+} ; z\right)$ (where for each $k, \beta_{k}^{+}$is isotopic to $\beta_{k}^{\prime}$ and intersects $\beta_{k}$ transversely in two points) such that for each $\mathbf{x} \in \mathbb{T}_{\boldsymbol{\alpha}} \cap \mathbb{T}_{\boldsymbol{\beta}}$, there is a 3-gon class $\psi_{g}^{+} \in \pi_{2}\left(\mathbf{x}, \boldsymbol{\theta}_{\boldsymbol{\beta} \boldsymbol{\beta}^{+}}, \mathbf{y}^{+}\right)$with $\mu\left(\psi_{g}^{+}\right)=0, \psi_{g}^{+} \cap \nabla=\emptyset$, and $n_{z}\left(\psi_{g}^{+}\right)=0$, where $\mathbf{y}^{+} \in \mathbb{T}_{\boldsymbol{\beta}} \cap \mathbb{T}_{\boldsymbol{\beta}^{+}}$is the nearest neighbor to $g(\mathbf{x})$.

(b) There is a Heegaard triple-diagram $\left(\Sigma ; \boldsymbol{\alpha} ; \boldsymbol{\beta}^{-} ; \boldsymbol{\beta} ; z\right)$ (where for each $k, \beta_{k}^{-}$is isotopic to $\beta_{k}^{\prime}$ and intersects $\beta_{k}$ transversely in two points) such that for each $\mathbf{x} \in \mathbb{T}_{\boldsymbol{\alpha}} \cap \mathbb{T}_{\boldsymbol{\beta}}^{\prime}$, there is a 3-gon class $\psi_{g}^{-} \in \pi_{2}\left(\mathbf{y}^{-}, \boldsymbol{\theta}_{\boldsymbol{\beta}^{-} \boldsymbol{\beta}}, \mathbf{x}\right)$ with $\mu\left(\psi_{g}^{-}\right)=0, \psi_{g}^{-} \cap \nabla=\emptyset$, and $n_{z}\left(\psi_{g}^{-}\right)=0$, where $\mathbf{y}^{-} \in \mathbb{T}_{\boldsymbol{\beta}} \cap \mathbb{T}_{\boldsymbol{\beta}^{-}}$is the nearest neighbor to $g(\mathbf{x})$.

Remark 5.0.5. Recall that when constructing chain homotopies associated to triangle-counting chain homotopy equivalences in Section 2.1.2, we composed them with nearest neighbor maps so that compositions were honestly chain-homotopic to identity maps.

For instance, if $(\Sigma ; \boldsymbol{\alpha} ; \boldsymbol{\beta} ; z)$ and $\left(\Sigma ; \boldsymbol{\alpha}^{\prime} ; \boldsymbol{\beta} ; z\right)$ are two admissible pointed Heegaard diagrams such that $\boldsymbol{\alpha}^{\prime}$ is obtained from $\boldsymbol{\alpha}$ via a pointed isotopy or handle slide, then

$$
\begin{aligned}
& \widehat{f}_{\boldsymbol{\alpha} \boldsymbol{\alpha}^{\prime} \boldsymbol{\beta}}\left(\boldsymbol{\theta}_{\boldsymbol{\alpha} \boldsymbol{\alpha}^{\prime}} \otimes \widehat{f}_{\boldsymbol{\alpha}^{\prime} \boldsymbol{\alpha} \boldsymbol{\beta}}\left(\boldsymbol{\theta}_{\boldsymbol{\alpha}^{\prime} \boldsymbol{\alpha}} \otimes \cdot\right)\right)-i d_{\widehat{C F}\left(\mathcal{H}_{\boldsymbol{\alpha} \boldsymbol{\beta}}\right)}=\widehat{\partial} H+H \widehat{\partial} \quad \text { and } \\
& \widehat{f}_{\boldsymbol{\alpha}^{\prime} \boldsymbol{\alpha} \boldsymbol{\beta}}\left(\boldsymbol{\theta}_{\boldsymbol{\alpha}^{\prime} \boldsymbol{\alpha}} \otimes \widehat{f}_{\boldsymbol{\alpha} \boldsymbol{\alpha}^{\prime} \boldsymbol{\beta}}\left(\boldsymbol{\theta}_{\boldsymbol{\alpha} \boldsymbol{\alpha}^{\prime}} \otimes \cdot\right)\right)-i d_{\widehat{C F}\left(\mathcal{H}_{\boldsymbol{\alpha}^{\prime} \boldsymbol{\beta}}\right)}=\widehat{\partial} G+G \widehat{\partial}
\end{aligned}
$$

where $H$ and $G$ are given by the expressions in equation (2.2) appearing in Remark 2.1.7.

Lemma 5.0.6. Let $\mathcal{H}=(\Sigma ; \boldsymbol{\alpha} ; \boldsymbol{\beta} ; z)$ and $\mathcal{H}^{\prime}=\left(\Sigma ; \boldsymbol{\alpha}^{\prime} ; \boldsymbol{\beta} ; z\right)$ be two admissible pointed Heegaard diagrams for the manifold $\Sigma(K) \#\left(S^{2} \times S^{1}\right)$ which are obtained from braids $b$ and $b^{\prime}$ (possibly after Heegaard stabilization). Assume that there is a sequence of pointed isotopies or handeslides

$$
\mathcal{H}=\mathcal{H}^{0} \rightarrow \mathcal{H}^{1} \rightarrow \ldots \rightarrow \mathcal{H}^{n}=\mathcal{H}^{\prime}
$$

where each pointed Heegaard diagram $\mathcal{H}^{k}:=\left(\Sigma ; \boldsymbol{\alpha}^{k} ; \boldsymbol{\beta}^{k} ; z\right)$ is admissible.

Also, let $g=g^{n} \circ \cdots \circ g^{1}$ be a composition of triangle injections

$$
g^{k}: \mathbb{T}_{\boldsymbol{\alpha}^{(k-1)}} \cap \mathbb{T}_{\boldsymbol{\beta}^{(k-1)}} \rightarrow \mathbb{T}_{\boldsymbol{\alpha}^{k}} \cap \mathbb{T}_{\boldsymbol{\beta}^{k}}
$$

and assume that $R(g(\mathbf{x}))=R(\mathbf{x})$ for each $\mathbf{x} \in \mathbb{T}_{\boldsymbol{\alpha}} \cap \mathbb{T}_{\boldsymbol{\beta}}$. Now for $1 \leq$ $k \leq n$, let $f^{k}: \widehat{C F}\left(\mathcal{H}^{(k-1)}\right) \rightarrow \widehat{C F}\left(\mathcal{H}^{k}\right)$ denote the 3-gon-counting chain homotopy equivalence induced by the $k^{\text {th }}$ Heegaard move in the sequence, let $h^{k}: \widehat{C F}\left(\mathcal{H}^{k}\right) \rightarrow \widehat{C F}\left(\mathcal{H}^{(k-1)}\right)$ denote its homotopy inverse, and let $H^{k}$ : $\widehat{C F}\left(\mathcal{H}^{(k-1)}\right) \rightarrow \widehat{C F}\left(\mathcal{H}^{(k-1)}\right)$ and $G^{k}: \widehat{C F}\left(\mathcal{H}^{k}\right) \rightarrow \widehat{C F}\left(\mathcal{H}^{k}\right)$ be associated homotopies as described in Remark 5.0.5. 
Now let $H: \widehat{C F}(\mathcal{H}) \rightarrow \widehat{C F}(\mathcal{H})$ and $G: \widehat{C F}\left(\mathcal{H}^{\prime}\right) \rightarrow \widehat{C F}\left(\mathcal{H}^{\prime}\right)$ be given by

$$
\begin{aligned}
H & :=H^{1}+\sum_{i=1}^{n-1}\left(h^{1} \circ \cdots \circ h^{i}\right) \circ H^{i+1} \circ\left(f^{i} \circ \cdots \circ f^{1}\right) \quad \text { and } \\
G & :=G^{n}+\sum_{i=1}^{n-1}\left(f^{n} \circ \cdots \circ f^{(i+1)}\right) \circ G^{i} \circ\left(h^{(i+1)} \circ \cdots \circ h^{n}\right),
\end{aligned}
$$

so that

$$
h f-i d_{\widehat{C F}(\mathcal{H})}=\widehat{\partial} H+H \widehat{\partial} \quad \text { and } \quad f h-i d_{\widehat{C F}\left(\mathcal{H}^{\prime}\right)}=\widehat{\partial} G+G \widehat{\partial} .
$$

Then for each torsion $\mathfrak{s} \in \operatorname{Spin}^{c}\left(\Sigma(K) \#\left(S^{2} \times S^{1}\right)\right)$, the following hold:

(i) If $\mathbf{y} \in \mathbb{T}_{\boldsymbol{\alpha}^{\prime}} \cap \mathbb{T}_{\boldsymbol{\beta}^{\prime}}$ is a term in the sum $f(\mathbf{x})$ for some $\mathbf{x} \in \mathfrak{U}_{\mathfrak{s}} \subset \mathbb{T}_{\boldsymbol{\alpha}} \cap \mathbb{T}_{\boldsymbol{\beta}}$ and if $\mathbf{w} \in \mathbb{T}_{\boldsymbol{\alpha}} \cap \mathbb{T}_{\boldsymbol{\beta}}$ is a term in the sum $h(\mathbf{z})$ for some $\mathbf{z} \in \mathfrak{U}_{\mathfrak{s}}^{\prime} \subset$ $\mathbb{T}_{\boldsymbol{\alpha}^{\prime}} \cap \mathbb{T}_{\boldsymbol{\beta}^{\prime}}$, then $\rho(\mathbf{y}) \leq \rho(\mathbf{x})$ and $\rho(\mathbf{w}) \leq \rho(\mathbf{z})$.

(ii) If $\mathbf{y} \in \mathbb{T}_{\boldsymbol{\alpha}} \cap \mathbb{T}_{\boldsymbol{\beta}}$ is a term in the sum $H(\mathbf{x})$ for some $\mathbf{x} \in \mathfrak{U}_{\mathfrak{s}} \subset \mathbb{T}_{\boldsymbol{\alpha}} \cap \mathbb{T}_{\boldsymbol{\beta}}$ and if $\mathbf{w} \in \mathbb{T}_{\boldsymbol{\alpha}^{\prime}} \cap \mathbb{T}_{\boldsymbol{\beta}^{\prime}}$ is a term in the sum $G(\mathbf{z})$ for some $\mathbf{z} \in \mathfrak{U}_{\mathfrak{s}}^{\prime} \subset$ $\mathbb{T}_{\boldsymbol{\alpha}^{\prime}} \cap \mathbb{T}_{\boldsymbol{\beta}^{\prime}}$, then $\rho(\mathbf{y}) \leq \rho(\mathbf{x})$ and $\rho(\mathbf{w}) \leq \rho(\mathbf{z})$.

Proof of part (i). Let $\mathbf{x} \in \mathfrak{U}_{\mathfrak{s}} \subset \mathbb{T}_{\boldsymbol{\alpha}} \cap \mathbb{T}_{\boldsymbol{\beta}}$ and let $\mathbf{y} \in \mathbb{T}_{\boldsymbol{\alpha}^{\prime}} \cap \mathbb{T}_{\boldsymbol{\beta}^{\prime}}$ be a term in the sum $f(\mathbf{x})$. Then there are two sequences

$$
\mathbf{x}=\mathbf{y}^{0}, \mathbf{y}^{1}, \ldots, \mathbf{y}^{n}=\mathbf{y} \text { and } \mathbf{x}=\mathbf{x}^{0}, \mathbf{x}^{1}, \ldots, \mathbf{x}^{n}=g(\mathbf{x})
$$

such that $\mathbf{y}^{j}, \mathbf{x}^{j} \in \mathbb{T}_{\boldsymbol{\alpha}^{j} \cap \boldsymbol{\beta}^{j}}, \mathbf{y}^{j}$ is a term in the sum $f^{j}\left(\mathbf{y}^{(j-1)}\right)$, and $\mathbf{x}^{j}=$ $g^{j}\left(\mathbf{x}^{(j-1)}\right)$ for each $j$.

We proceed by induction. Without loss of generality, assume that the $j^{\text {th }}$ Heegaard move in the sequence is one among the $\boldsymbol{\alpha}$ curves. i.e., that $\boldsymbol{\beta}^{j}=\boldsymbol{\beta}^{(j-1)}$ and $g^{j}$ is a $\boldsymbol{\alpha}$-triangle injection.

Recall that we have a class $\psi_{f^{j}} \in \pi_{2}\left(\boldsymbol{\theta}_{\boldsymbol{\alpha}^{j} \boldsymbol{\alpha}^{(j-1)}}, \mathbf{y}^{(j-1)}, \mathbf{y}^{j}\right)$ with a pseudoholomorphic representative such that $\mu\left(\psi_{f^{j}}\right)=n_{z}\left(\psi_{f^{j}}\right)=0$. Furthermore, the triangle injection $g^{j}$ provides a class $\psi_{g^{j}} \in \pi_{2}\left(\boldsymbol{\theta}_{\boldsymbol{\alpha}^{j} \boldsymbol{\alpha}^{(j-1)}}, \mathbf{x}^{(j-1)}, \mathbf{x}^{j}\right)$ avoiding $\nabla$ such that $\mu\left(\psi_{g^{j}}\right)=n_{z}\left(\psi_{g^{j}}\right)=0$.

Assume that we have already obtained a 2-gon class $\eta_{\boldsymbol{\alpha}^{(j-1)} \boldsymbol{\beta}} \in \pi_{2}$ $\left(\mathbf{x}^{(j-1)}, \mathbf{y}^{(j-1)}\right)$ with $\mu\left(\eta_{\boldsymbol{\alpha}^{(j-1)} \boldsymbol{\beta}}\right)=n_{z}\left(\eta_{\boldsymbol{\alpha}^{(j-1)} \boldsymbol{\beta}}\right)=\left[\eta_{\boldsymbol{\alpha}^{(j-1)} \boldsymbol{\beta}}\right] \cdot[\nabla]=0$. For the base case, we note that $\mathbf{x}^{0}=\mathbf{y}^{0}=\mathbf{x}$ and we let $\eta_{\boldsymbol{\alpha}^{0} \boldsymbol{\beta}} \in \pi_{2}(\mathbf{x}, \mathbf{x})$ be the class with trivial domain. Then the concatenation $\widetilde{\psi}_{j}:=\left(\eta_{\boldsymbol{\alpha}^{(j-1)} \boldsymbol{\alpha}}+\psi_{f^{j}}\right)$ is an element of $\pi_{2}\left(\boldsymbol{\theta}_{\boldsymbol{\alpha}^{j} \boldsymbol{\alpha}^{(j-1)}}, \mathbf{x}^{(j-1)}, \mathbf{y}^{j}\right)$. The classes $\widetilde{\psi}_{j}$ and $\psi_{g^{j}}$ are $\operatorname{Spin}^{{ }^{-}-}$ equivalent, and thus there are 2-gons $\eta_{(j-1)} \in \pi_{2}\left(\mathbf{x}^{(j-1)}, \mathbf{x}^{(j-1)}\right), \eta_{\boldsymbol{\alpha}^{j} \boldsymbol{\beta}} \in$ $\pi_{2}\left(\mathbf{x}^{j}, \mathbf{y}^{j}\right)$, and $\eta_{\boldsymbol{\alpha}^{j} \boldsymbol{\alpha}^{(j-1)}} \in \pi_{2}\left(\boldsymbol{\theta}_{\boldsymbol{\alpha}^{j} \boldsymbol{\alpha}^{(j-1)}}, \boldsymbol{\theta}_{\boldsymbol{\alpha}^{j} \boldsymbol{\alpha}^{(j-1)}}\right)$ such that

$$
\widetilde{\psi}_{j}=\psi_{g^{j}}+\eta_{(j-1)}+\eta_{\boldsymbol{\alpha}^{j} \boldsymbol{\beta}}+\eta_{\boldsymbol{\alpha}^{j} \boldsymbol{\alpha}^{(j-1)}} .
$$


Now $\mathcal{D}\left(\eta_{(j-1)}\right)+\mathcal{D}\left(\eta_{\boldsymbol{\alpha}^{j} \boldsymbol{\alpha}^{(j-1)}}\right)$ can be viewed as a triply-periodic element of $\Pi_{\boldsymbol{\alpha}^{(j-1)} \boldsymbol{\beta}^{j} \boldsymbol{\alpha}^{j}}$, and so by Proposition 2.2.2 can be written as a sum of the doubly-periodic domains $\mathcal{D}_{k}^{\boldsymbol{\alpha}^{(j-1)} \boldsymbol{\alpha}^{j}} \in \Pi_{\boldsymbol{\alpha}^{(j-1)} \boldsymbol{\alpha}^{j}}$. For each $k$, let $\eta_{k}^{j} \in$ $\pi_{2}\left(\boldsymbol{\theta}_{\boldsymbol{\alpha}^{j} \boldsymbol{\alpha}^{(j-1)}}, \boldsymbol{\theta}_{\boldsymbol{\alpha}^{j} \boldsymbol{\alpha}^{(j-1)}}\right)$ denote the 2-gon whose domain is $\mathcal{D}_{k}^{\boldsymbol{\alpha}^{(j-1)} \boldsymbol{\alpha}^{j}}$ - by Lemma 4.5.1, $\eta_{k}^{j}$ avoids the anti-diagonal $\nabla$ (and of course the basepoint). The class $\xi_{j}:=\eta_{(j-1)}+\eta_{\boldsymbol{\alpha}^{j} \boldsymbol{\alpha}^{(j-1)}}$ can be written as a sum of elements of classes in the set $\left\{\eta_{1}^{j}, \ldots, \eta_{g}^{j}\right\}$, and thus $\left[\xi_{j}\right] \cdot[\nabla]=n_{z}\left(\xi_{j}\right)=0$. Thus,

$$
\begin{aligned}
\mu\left(\eta_{\boldsymbol{\alpha}^{j} \boldsymbol{\beta}}\right) & =\mu\left(\psi_{f^{j}}+\eta_{\boldsymbol{\alpha}^{(j-1)} \boldsymbol{\alpha}}\right)-\mu\left(\psi_{g^{j}}\right)-\mu\left(\xi_{j}\right)=0, \\
{\left[\eta_{\boldsymbol{\alpha}^{j} \boldsymbol{\beta}}\right] \cdot[\nabla] } & =\left[\psi_{f^{j}}+\eta_{\boldsymbol{\alpha}^{(j-1)} \boldsymbol{\alpha}}\right] \cdot[\nabla]-\left[\psi_{g^{j}}\right] \cdot[\nabla]-\left[\xi_{j}\right] \cdot[\nabla] \\
& =\left[\psi_{f^{j}}\right] \cdot[\nabla] \geq 0, \quad \text { and } \quad n_{z}\left(\eta_{\boldsymbol{\alpha}^{j} \boldsymbol{\beta}}\right)=0 .
\end{aligned}
$$

where the last inequality follows from the fact that $\psi_{f^{j}}$ has a pseudoholomorphic representative. After $n$ steps, we obtain the required class $\eta_{\boldsymbol{\alpha}^{n} \boldsymbol{\beta}} \in \pi_{2}(g(\mathbf{x}), \mathbf{y})$.

Now since $R(\mathbf{x})=R(g(\mathbf{x})) \geq R(\mathbf{y}), n$ iterations of Lemma 5.0.3 give that

$$
\rho(\mathbf{x})=R(\mathbf{x})-\widetilde{g r}(\mathbf{x}) \geq R(\mathbf{y})-\widetilde{g r}(\mathbf{x})=R(\mathbf{y})-\widetilde{g r}(\mathbf{y})=\rho(\mathbf{y}) .
$$

On the other hand, let $\mathbf{z} \in \mathfrak{U}_{\mathfrak{s}}^{\prime} \subset \mathbb{T}_{\boldsymbol{\alpha}^{\prime}} \cap \mathbb{T}_{\boldsymbol{\beta}^{\prime}}$ and let $\mathbf{w}$ be a term in the sum $\left(h^{1} \circ \cdots \circ h^{n}\right)(\mathbf{z})$. A similar induction argument provides a 2-gon class $\left.\eta \in \pi_{2}(g(\mathbf{w}), \mathbf{z})\right)$ such that

$$
\mu(\eta)=n_{z}(\eta)=0 \quad \text { and } \quad[\eta] \cdot[\nabla] \leq 0 .
$$

Now we have that $R(\mathbf{w})=R(g(\mathbf{w})) \leq R(\mathbf{z})$, and so

$$
\rho(\mathbf{w})=R(\mathbf{w})-\widetilde{g r}(\mathbf{w})=R(\mathbf{z})-\widetilde{g r}(\mathbf{w}) \leq R(\mathbf{z})-\widetilde{g r}(\mathbf{z})=\rho(\mathbf{z})
$$

Proof of part (ii). Without loss of generality, assume that the $k^{\text {th }}$ Heegaard move is among the $\boldsymbol{\alpha}$ curves (so that $\boldsymbol{\beta}^{k}=\boldsymbol{\beta}^{(k-1)}$ and $g^{k}$ is a $\boldsymbol{\alpha}$-triangle injection). We work in the pointed Heegaard quadruple-diagram $\left(\Sigma ; \widetilde{\boldsymbol{\alpha}}^{(k-1)} ; \boldsymbol{\alpha}^{k} ; \boldsymbol{\alpha}^{(k-1)} ; \boldsymbol{\beta}^{k} ; z\right)$, where $\widetilde{\boldsymbol{\alpha}}^{(k-1)}$ is a set of attaching circles obtained from $\boldsymbol{\alpha}^{(k-1)}$ by a small admissible isotopy. Suppose that $\mathbf{y} \in$ $\mathbb{T}_{\boldsymbol{\alpha}} \cap \mathbb{T}_{\boldsymbol{\beta}}$ appears as a term in the sum

$$
\left(h^{1} \circ \cdots \circ h^{k}\right) \circ H^{(k+1)} \circ\left(f^{k} \circ \cdots \circ f^{1}\right)(\mathbf{x}),
$$

then $\rho(\mathbf{y}) \leq \rho(\mathbf{x})$.

Then there are $\mathbf{z}^{j}, \mathbf{u}^{j} \in \mathbb{T}_{\boldsymbol{\alpha}^{j}} \cap \mathbb{T}_{\boldsymbol{\beta}^{j}}$ for $j=0, \ldots, k$ with $\mathbf{z}^{0}=\mathbf{x}, \mathbf{u}^{0}=\mathbf{y}$, $\mathbf{z}^{i}$ a term in $f^{i}\left(\mathbf{z}^{(i-1)}\right), \mathbf{u}^{k}$ a term in $H^{(k+1)}\left(\mathbf{z}^{k}\right)$, and $\mathbf{u}^{(i-1)}$ a term in $h^{i}\left(\mathbf{u}^{i}\right)$ for $i=1, \ldots, k$.

Recall that

$$
H^{(k+1)}:=N_{\widetilde{\boldsymbol{\alpha}}^{k} \boldsymbol{\alpha}^{k}} \circ \widetilde{H}^{(k+1)},
$$




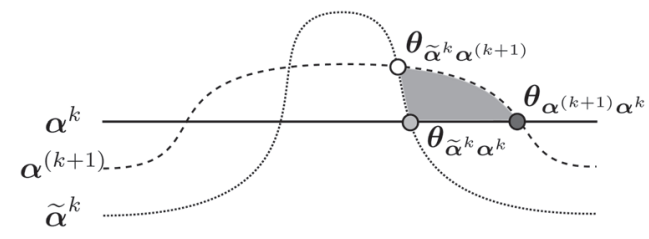

Figure 14. A component of the domain of the 3-gon $\psi_{\theta} \in$ $\pi_{2}\left(\boldsymbol{\theta}_{\widetilde{\boldsymbol{\alpha}}^{k} \boldsymbol{\alpha}^{(k+1)}}, \boldsymbol{\theta}_{\boldsymbol{\alpha}^{(k+1)} \boldsymbol{\alpha}^{k}}, \boldsymbol{\theta}_{\widetilde{\boldsymbol{\alpha}}^{k} \boldsymbol{\alpha}^{k}}\right)$.

where $N_{\widetilde{\boldsymbol{\alpha}}^{k} \boldsymbol{\alpha}^{k}}: \widetilde{\mathbf{v}} \rightarrow \mathbf{v}$ is the nearest neighbor isomorphism and

$$
\widetilde{H}^{(k+1)}:=\widehat{h}_{\widetilde{\boldsymbol{\alpha}}^{k} \boldsymbol{\alpha}^{(k+1)} \boldsymbol{\alpha}^{k} \boldsymbol{\beta}}\left(\boldsymbol{\theta}_{\widetilde{\boldsymbol{\alpha}}^{k} \boldsymbol{\alpha}^{(k+1)}} \otimes \boldsymbol{\theta}_{\boldsymbol{\alpha}^{(k+1)} \boldsymbol{\alpha}^{k}} \otimes \cdot\right)
$$

is a map counting pseudo-holomorphic representatives of 4-gon classes. There is then such a 4-gon class $\sigma \in \pi_{2}\left(\boldsymbol{\theta}_{\widetilde{\boldsymbol{\alpha}}^{k} \boldsymbol{\alpha}^{(k+1)}}, \boldsymbol{\theta}_{\boldsymbol{\alpha}^{(k+1)} \boldsymbol{\alpha}^{k}}, \mathbf{z}^{k}, \widetilde{\mathbf{u}}^{k}\right)$ such that $\mu(\sigma)=-1$ and $[\sigma] \cdot[\nabla] \geq 0$.

Now for $0 \leq j \leq k, \mathfrak{s}_{z}\left(\mathbf{u}^{j}\right)=\mathfrak{s}_{z}\left(\mathbf{z}^{j}\right)$ and by Lemma 5.0.3,

$$
\widetilde{g r}\left(\mathbf{z}^{j}\right)-\widetilde{g r}\left(\mathbf{u}^{j}\right)=\widetilde{g r}\left(\mathbf{z}^{k}\right)-\widetilde{g r}\left(\mathbf{u}^{k}\right)=\widetilde{g r}\left(\mathbf{z}^{k}\right)-\widetilde{g r}\left(\widetilde{\mathbf{u}^{k}}\right)=\mu(\sigma)=-1
$$

As a result, there are 2-gon classes $\zeta^{j} \in \pi_{2}\left(\mathbf{z}^{j}, \mathbf{u}^{j}\right)$ such that $\mu\left(\zeta^{j}\right)=-1$.

There are also index-zero 3-gon classes $\widetilde{\psi} \in \pi_{2}\left(\boldsymbol{\theta}_{\widetilde{\boldsymbol{\alpha}}^{k} \boldsymbol{\alpha}^{k}}, \mathbf{u}^{k}, \widetilde{\mathbf{u}}^{k}\right.$ ) (with domain components as in Figure 13) and $\psi_{\theta} \in \pi_{2}\left(\boldsymbol{\theta}_{\widetilde{\boldsymbol{\alpha}}^{k} \boldsymbol{\alpha}^{(k+1)}}\right.$, $\boldsymbol{\theta}_{\boldsymbol{\alpha}^{(k+1)} \boldsymbol{\alpha}^{k}}, \boldsymbol{\theta}_{\widetilde{\boldsymbol{\alpha}}^{k} \boldsymbol{\alpha}^{k}}$ ) (with domain components as in Figure 14); it can be arranged that these classes have small domains, so that $\widetilde{\psi} \cap \nabla=\psi_{\theta} \cap \nabla=\emptyset$.

Note that $\widetilde{\psi}+\psi_{\theta}+\zeta^{k} \in \pi_{2}\left(\boldsymbol{\theta}_{\widetilde{\boldsymbol{\alpha}}^{k} \boldsymbol{\alpha}^{(k+1)}}, \boldsymbol{\theta}_{\boldsymbol{\alpha}^{(k+1)} \boldsymbol{\alpha}^{k}}, \mathbf{z}^{k}, \widetilde{\mathbf{u}}^{k}\right)$, and so there is some 4-gon $\eta$ with quadruply-periodic domain such that $\widetilde{\psi}+\psi_{\theta}+\zeta^{k}=\sigma+\eta$.

But recall that $\eta$ can be written as the concatenation of 2-gons which avoid the anti-diagonal $\nabla$ and the basepoint $z$, and so

$\left[\zeta^{k}\right] \cdot[\nabla]=[\sigma] \cdot[\nabla]+[\eta] \cdot[\nabla]-[\widetilde{\psi}] \cdot[\nabla]-\left[\psi_{\theta}\right] \cdot[\nabla]=[\sigma] \cdot[\nabla]+0-0-0 \geq 0$.

Consider the sequence $\mathbf{x}^{0}:=\mathbf{x}=\mathbf{z}^{0}, \mathbf{x}^{1}, \ldots, \mathbf{x}^{k}$ with $\mathbf{x}^{j}=g^{j}\left(\mathbf{x}^{(j-1)}\right)$ for each $j$ (here $\mathbf{x}^{j} \in \mathbb{T}_{\boldsymbol{\alpha}^{j}} \cap \mathbb{T}_{\boldsymbol{\beta}^{j}}$ ). Recall that the proof of the first part of this lemma provided 2-gon classes $\phi^{i} \in \pi_{2}\left(\mathbf{x}^{i}, \mathbf{z}^{i}\right)$ with $\mu\left(\phi^{i}\right)=0$ and $\left[\phi^{i}\right] \cdot[\nabla] \geq 0$ for $i=1, \ldots, k$. We will show that for each $i$ with $1 \leq i \leq k$

$$
\left[\zeta^{(i-1)}\right] \cdot[\nabla]+\left[\phi^{(i-1)}\right] \cdot[\nabla] \geq\left[\zeta^{i}\right] \cdot[\nabla]+\left[\phi^{i}\right] \cdot[\nabla],
$$

where $\phi^{0}$ denotes the class with trivial domain connecting $\mathbf{x}^{0}=\mathbf{z}^{0}=\mathbf{x}$ to itself.

Fix $i$ with $1 \leq i \leq k$ and assume (without loss of generality) that the $i^{\text {th }}$ Heegaard move is among the $\alpha$ curves. The triangle injection $g^{i}$ provides a class $\psi_{g^{i}} \in \pi_{2}\left(\boldsymbol{\theta}_{\boldsymbol{\alpha}^{(i-1)} \boldsymbol{\alpha}^{i}}, \mathbf{x}^{i}, \mathbf{x}^{(i-1)}\right)$ such that $\mu\left(\psi_{g^{i}}\right)=n_{z}\left(\psi_{g^{i}}\right)=0$ and $\left[\psi_{g^{i}}\right] \cdot[\nabla]=0$. Additionally, there is a class $\psi_{h^{i}} \in \pi_{2}\left(\boldsymbol{\theta}_{\boldsymbol{\alpha}^{(i-1)} \boldsymbol{\alpha}^{i}}, \mathbf{u}^{i}, \mathbf{u}^{(i-1)}\right)$ with pseudo-holomorphic representative such that $\mu\left(\psi_{h^{i}}\right)=n_{z}\left(\psi_{h^{i}}\right)=0$. 
Note that $\psi_{g^{i}}+\zeta^{(i-1)}+\phi^{(i-1)}, \phi^{i}+\zeta^{i}+\psi_{h^{i}} \in \pi_{2}\left(\boldsymbol{\theta}_{\boldsymbol{\alpha}^{(i-1)} \boldsymbol{\alpha}^{i}}, \mathbf{x}^{i}, \mathbf{u}^{(i-1)}\right)$. Then there is some 3 -gon $\eta^{i}$ with triply-periodic domain such that

$$
\psi_{g^{i}}+\zeta^{(i-1)}+\phi^{(i-1)}=\phi^{i}+\zeta^{i}+\psi_{h^{i}}+\eta^{i} .
$$

Since $\left[\psi_{g^{i}}\right] \cdot[\nabla]=\left[\eta^{i}\right] \cdot[\nabla]=0$ and $\left[\psi_{h^{i}}\right] \cdot[\nabla] \geq 0$, equation (5.2) holds. Therefore,

$$
\left[\zeta^{0}\right] \cdot[\nabla] \geq\left[\zeta^{1}\right] \cdot[\nabla]+\left[\phi^{1}\right] \cdot[\nabla] \geq \cdots \geq\left[\zeta^{k}\right] \cdot[\nabla]+\left[\phi^{k}\right] \cdot[\nabla] \geq 0 .
$$

On the other hand, given some $\mathbf{z}, \mathbf{w} \in \mathbb{T}_{\boldsymbol{\alpha}^{\prime}} \cap \mathbb{T}_{\boldsymbol{\beta}^{\prime}}$ such that $\mathbf{w}$ is a term in $G(\mathbf{z})$, a similar argument produces a 2-gon class $\xi^{0} \in \pi_{2}(\mathbf{z}, \mathbf{w})$ such that $\left[\xi^{0}\right] \cdot[\nabla] \geq 0$ and $\mu\left(\xi^{0}\right)=-1$.

We formulate the following restatement of Lemma 5.0.6.

Corollary 5.0.7. Let $\mathcal{H}=(\Sigma ; \boldsymbol{\alpha} ; \boldsymbol{\beta} ; z)$ and $\mathcal{H}^{\prime}=\left(\Sigma ; \boldsymbol{\alpha}^{\prime} ; \boldsymbol{\beta}^{\prime} ; z\right)$ be two admissible pointed Heegaard diagrams for the manifold $\Sigma(K) \#\left(S^{2} \times S^{1}\right)$ which are obtained from braids $b$ and $b^{\prime}$ (possibly after Heegaard stabilization), and related by handleslides and isotopies in the sense of Lemma 5.0.6, where the intermediate pointed Heegaard diagrams are all admissible. Assume also that there are triangle injections corresponding to each of these Heegaard moves such that their composition $g: \mathbb{T}_{\boldsymbol{\alpha}} \cap \mathbb{T}_{\boldsymbol{\beta}} \rightarrow \mathbb{T}_{\boldsymbol{\alpha}^{\prime}} \cap \mathbb{T}_{\boldsymbol{\beta}^{\prime}}$ satisfies $R(g(\mathbf{x}))=$ $R(\mathbf{x})$ for all $\mathbf{x} \in \mathbb{T}_{\boldsymbol{\alpha}} \cap \mathbb{T}_{\boldsymbol{\beta}}$. Then for each $\mathfrak{s} \in \operatorname{Spin}^{c}\left(\Sigma(K) \#\left(S^{2} \times S^{1}\right)\right)$ torsion, the following hold:

(i) The composition $f: \widehat{C F}(\mathcal{H}, \mathfrak{s}) \rightarrow \widehat{C F}\left(\mathcal{H}^{\prime}, \mathfrak{s}\right)$ of chain homotopy equivalences induced by the moves and its homotopy inverse $h: \widehat{C F}\left(\mathcal{H}^{\prime}, \mathfrak{s}\right) \rightarrow$ $\widehat{C F}(\mathcal{H}, \mathfrak{s})$ are $\rho$-filtered chain maps.

(ii) The homotopies $H$ from $g \circ f$ to $i d_{\widehat{C F}(\mathcal{H}, \mathfrak{s})}$ and $G$ from $f \circ g$ to $i d_{\widehat{C F}\left(\mathcal{H}^{\prime}, \mathfrak{s}\right)}$ are $\rho$-filtered chain homotopies.

In particular, the $\rho$-filtered complexes $\widehat{C F}(\mathcal{H}, \mathfrak{s})$ and $\widehat{C F}\left(\mathcal{H}^{\prime}, \mathfrak{s}\right)$ have the same filtered chain homotopy type.

We turn to a few lemmas which will later allow us to restrict our attention to multiplication of braids in $B_{2 n}$ by elements of $K_{2 n}$ on the right side only. Note first that the symplectic automorphism induced by the braid $b \in B_{2 n}$ on the punctured disk induces a symplectic automorphism $f_{b}: \operatorname{Sym}^{n}(\Sigma) \rightarrow$ $\operatorname{Sym}^{n}(\Sigma)$.

One can see that there is an induced graded symplectic automorphism $\widetilde{f}_{b}$ with respect to gradings provided by the volume form.

Lemma 5.0.8. Let $f_{b}: \operatorname{Sym}^{n}(\Sigma) \rightarrow \operatorname{Sym}^{n}(\Sigma)$ be the automorphism discussed above and let $\nabla \subset \operatorname{Sym}^{n}(\Sigma)$ denote the anti-diagonal. Then $f_{b}(\nabla)=\nabla$. 
Proof. Let $\mathbf{x} \in \nabla$. Then $\mathbf{x}$ contains components $\left(u_{1}, z_{1}\right)$ and $\left(u_{2}, z_{2}\right)$ such that $z_{2}=z_{1}$ and $u_{2}=-u_{1}$. Suppose that $u_{2} \neq 0$. Then $f_{b}(\mathbf{x})=\left(v_{k}, w_{k}\right)$ contains components $\left(v_{1}, w_{1}\right)=f_{b}\left(u_{1}, z_{1}\right)$ and $\left(v_{2}, w_{2}\right)=f_{b}\left(u_{2}, z_{2}\right)$. Since $f$ is induced by a map on the punctured disk, we have that $w_{2}=w_{1}$. Therefore, $\left(v_{2}\right)^{2}=\left(v_{1}\right)^{2}$ and so $v_{2}= \pm v_{1}$.

Now let $\mathbf{x}^{\prime}=\left(u_{k}^{\prime}, z_{k}^{\prime}\right) \in \Delta$ be such that $u_{2}^{\prime}=-u_{2}, u_{j}^{\prime}=u_{j}$ for $j \neq 2$, and $z_{j}^{\prime}=z_{j}$ for $j=1, \ldots, n$. Then if $f_{b}\left(\mathbf{x}^{\prime}\right)=\left(v_{k}^{\prime}, w_{k}^{\prime}\right)$, we then have that $v_{j}^{\prime}=v_{j}$ for $j \neq 2$ and $w_{j}^{\prime}=w_{j}$ for $j=1, \ldots, n$. Further, $v_{2}^{\prime}=v_{1}^{\prime}=v_{1}= \pm v_{2}$. But $\mathbf{x} \neq \mathbf{x}^{\prime}$, so $v_{2}^{\prime} \neq v_{2}$ and thus $v_{2}=-v_{1}$.

Now suppose that $u_{2}=u_{1}=0$. Then $z_{2}$ is a puncture point. However, a braid element diffeomorphism on the punctured disk fixes the set of punctures, and so $v_{2}=v_{1}=0$ also. So, $f_{b}(\mathbf{x}) \in \nabla$ in this case also.

One can similarly show that $f_{b}^{-1}(\nabla) \subset \nabla$.

As shorthand, let $\widetilde{f}_{b}\left(\mathbb{T}_{\boldsymbol{\alpha}}\right)$ be denoted by $b \mathbb{T}_{\boldsymbol{\alpha}}$ from now on. Since $R$ provides an absolute grading on $C F_{*}\left(\mathbb{T}_{\boldsymbol{\alpha}}, b \mathbb{T}_{\boldsymbol{\alpha}}\right)$, when computed inside $\operatorname{Sym}^{n}(\Sigma)-\nabla$, then one can define a grading $R^{*}$ on $C F^{-*}\left(\mathbb{T}_{\boldsymbol{\alpha}}, b \mathbb{T}_{\boldsymbol{\alpha}}\right)$ by letting $R^{*}\left(\mathbf{x}^{*}\right)=-R(\mathbf{x})$ for each $\mathbf{x} \in \mathbb{T}_{\boldsymbol{\alpha}} \cap b \mathbb{T}_{\boldsymbol{\alpha}}$.

Lemma 5.0.9. Let $b \in B_{2 n}$ be a braid. Then when computed inside $\operatorname{Sym}^{n}(\Sigma)-\nabla$, the complexes $C F_{*}\left(\mathbb{T}_{\boldsymbol{\alpha}}, b \mathbb{T}_{\boldsymbol{\alpha}}\right)$ and $C F^{-*}\left(\mathbb{T}_{\boldsymbol{\alpha}}, b^{-1} \mathbb{T}_{\boldsymbol{\alpha}}\right)$ are isomorphic as absolutely graded chain complexes equipped with the gradings $R$ and $R^{*}$, respectively.

Proof. The grading $\widetilde{R}$ arises as an absolute grading induced by gradings on totally-real submanifolds, and so as $\widetilde{R}$-graded complexes,

$$
C F_{*}\left(\mathbb{T}_{\boldsymbol{\alpha}}, b \mathbb{T}_{\boldsymbol{\alpha}}\right) \cong C F^{n-*}\left(b \mathbb{T}_{\boldsymbol{\alpha}}, \mathbb{T}_{\boldsymbol{\alpha}}\right) \cong C F^{n-*}\left(\mathbb{T}_{\boldsymbol{\alpha}}, b^{-1} \mathbb{T}_{\boldsymbol{\alpha}}\right) .
$$

But since $s_{R}\left(b^{-1}\right)+s_{R}(b)=-n$, the result follows.

When one computes these complexes inside all of $\operatorname{Sym}^{n}(\Sigma)$, recall that $C F^{-*}\left(\mathbb{T}_{\boldsymbol{\alpha}}, b \mathbb{T}_{\boldsymbol{\alpha}}\right)=\widehat{C F}\left(\mathcal{H}_{b}\right)$, where $\mathcal{H}_{b}$ is the admissible Heegaard diagram for $\Sigma(K) \#\left(S^{2} \times S^{1}\right)$ provided by Proposition 4.2.1 and $K$ is the closure of $b$. It is clear that $R^{*}$ provides a filtration on this complex.

Lemma 5.0.10. Let $b \in B_{2 n}$ be a braid and denote by $\mathcal{H}_{b}$ and $\mathcal{H}_{b^{-1}}$ the admissible Heegaard diagrams induced by the braid $b$ and $b^{-1}$, respectively. Then the complexes $\widehat{C F}_{*}\left(\mathcal{H}_{b^{-1}}\right)$ and $\widehat{C F}^{-*}\left(\mathcal{H}_{b}\right)$ are isomorphic as filtered chain complexes equipped with the filtrations $R$ and $R^{*}$, respectively.

Proof. When one extends the computation of the Floer complexes to all of $\operatorname{Sym}^{n}(\Sigma)$, the differentials may have additional terms which count classes of 2 -gons intersecting $\nabla$. By Lemma 5.0.8, the chain isomorphisms in the proof of Lemma 5.0.9 induce identifications between such classes which preserve intersection counts with $\nabla$. 


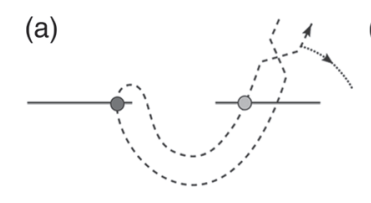

(b)

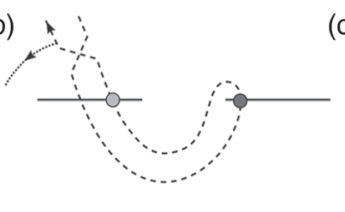

(c)

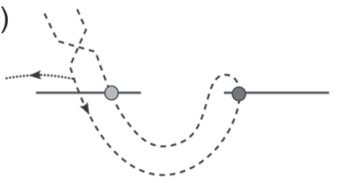

Figure 15. Local pictures of fork diagrams, where corresponding points are marked with matching dots. (a) The diagram $\mathcal{F}^{+}$, (b) $\widetilde{\mathcal{F}}$, the mirror of $\mathcal{F}^{+}$and (c) The diagram $\mathcal{F}^{-}$.

Now given a braid word $b=\sigma_{i_{1}}^{k_{1}} \ldots \sigma_{i_{m}}^{k_{m}} \in B_{2 n}$, let $-b$ denote the braid word $\sigma_{2 n-i_{1}}^{-k_{1}} \cdots \sigma_{2 n-i_{m}}^{-k_{m}} \in B_{2 n}$.

Lemma 5.0.11. Let $b \in B_{2 n}$ be a braid whose closure is the knot $K$, let $\mathfrak{s} \in$ $\operatorname{Spin}^{c}\left(\Sigma(K) \#\left(S^{2} \times S^{1}\right)\right)$ be torsion, and denote by $\mathcal{H}_{b}$ and $\mathcal{H}_{-b}$ the admissible Heegaard diagrams induced by the braids $b$ and $-b$, respectively. Then the complexes $\widehat{C F}_{*}\left(\mathcal{H}_{b}, \mathfrak{s}\right)$ and $\widehat{C F}^{-*}\left(\mathcal{H}_{-b}, \mathfrak{s}\right)$ are isomorphic as filtered chain complexes equipped with the filtrations $R$ and $R^{*}$, respectively.

Proof. Let $\mathcal{F}^{+}$and $\mathcal{F}^{-}$be the fork diagrams induced by $b$ and $-b$, respectively. Note that if the closure of $b$ is the knot $K$, then the closure of $-b$ is $-K$, the mirror image of $K$. Therefore, $\mathcal{H}_{ \pm b}$ is a Heegaard diagram for $\pm \Sigma(K) \#\left(S^{2} \times S^{1}\right)$. Let $\iota: \mathbb{C} \rightarrow \mathbb{C}$ be the map given by $z \mapsto-\bar{z}$, and let $\widetilde{\mathcal{F}}$ denote the "fork-like" diagram obtained by applying $\iota$ to $\mathcal{F}^{+}$. Note that if one ignores the handles, then $\widetilde{\mathcal{F}}$ is isotopic to $\mathcal{F}^{-}$. Figure 15 compares local pictures of these diagrams.

This map induces a diffeomorphism $\widehat{\iota}: \Sigma \rightarrow-\Sigma$, where $\mathcal{H}_{b}=$ $(\Sigma, \boldsymbol{\alpha}, \boldsymbol{\beta},+\infty)$ and $\mathcal{H}_{-b}=(-\Sigma, \boldsymbol{\alpha}, \boldsymbol{\beta},+\infty)$. Recall that for any closed, connected, oriented 3 -manifold $Y$, $\operatorname{Spin}^{c}(Y) \cong \operatorname{Spin}^{c}(-Y)$. For each torsion $\mathfrak{s} \in \operatorname{Spin}^{c}\left(\Sigma(K) \#\left(S^{2} \times S^{1}\right)\right)$, Ozsváth and Szabó described in [12] a natural chain isomorphism

$$
\Phi: \widehat{C F}_{*}\left(\Sigma(-K) \#\left(S^{2} \times S^{1}\right), \mathfrak{s}\right) \rightarrow \widehat{C F}^{-*}\left(\Sigma(K) \#\left(S^{2} \times S^{1}\right), \mathfrak{s}\right),
$$

which in our case is realized by $\Phi(\mathbf{x})=\left(\left\{\hat{\iota}^{-1}\left(x_{i}\right)\right\}\right)^{*}$ for each generator $\mathbf{x}$ for $\widehat{C F}_{*}\left(\Sigma(-K) \#\left(S^{2} \times S^{1}\right), \mathfrak{s}\right)$.

Figure 15 displays a suitably general local picture of the fork diagrams involved. Let $\mathbf{z}^{+}$be a generator in $\mathcal{F}^{+}$, let $\widetilde{\mathbf{z}}$ be the tuple in $\widetilde{\mathcal{F}}$ such that $\widetilde{z}_{j}=\iota\left(z_{j}^{+}\right)$, and let $\mathbf{z}^{-}$be the corresponding generator in $\mathcal{F}^{-}$. Define the functions $Q, P, T$, and $\widetilde{R}$ on $\widetilde{\mathcal{F}}$ in the obvious way. One can verify that

$$
\begin{gathered}
\left(P^{*}-Q^{*}\right)\left(z_{j}^{-}\right)=\left(P^{*}-Q^{*}\right)\left(\widetilde{z}_{j}\right)+1=-\left(P^{*}-Q^{*}\right)\left(z_{j}^{+}\right)+1, \\
\text { and so }(P-Q)\left(\mathbf{z}^{-}\right)=-(P-Q)\left(\mathbf{z}^{+}\right)+n .
\end{gathered}
$$


(a)

(b)

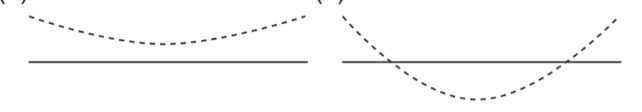

Figure 16. An isotopy in a fork diagram introducing two intersections between a $\alpha$ arc (solid) and a $\beta$ arc (dotted). (a) Before the isotopy and (b) After the isotopy.

Furthermore, $T\left(\mathbf{z}^{-}\right)=-T\left(\mathbf{z}^{+}\right)$, and

$$
\begin{aligned}
s_{R}\left(b^{-}, D^{-}\right) & =\frac{e\left(b^{-}\right)-w\left(D^{-}\right)-2 n}{4}=\frac{-e\left(b^{+}\right)+w\left(D^{+}\right)-2 n}{4} \\
& =-\frac{e\left(b^{+}\right)-w\left(D^{+}\right)-2 n}{4}-n .
\end{aligned}
$$

Therefore, we have that $R\left(\mathbf{z}^{-}\right)=-R\left(\mathbf{z}^{+}\right)$, and so both $\Phi$ and $\Phi^{-1}$ are filtered.

5.1. Fork diagram isotopy. The identification of a braid $b$ with its associated fork diagram is only defined up to isotopy of the fork diagram. We should verify the following:

Proposition 5.1.1. Let $\mathfrak{s} \in \operatorname{Spin}^{c}\left(\Sigma(K) \#\left(S^{2} \times S^{1}\right)\right)$ be torsion. Then the $\rho$-filtered chain homotopy type of $\widehat{C F}\left(\Sigma(K) \#\left(S^{2} \times S^{1}\right), \mathfrak{s}\right)$ is an invariant of the braid $b$.

Remark 5.1.2. The reader should note that in the original proof in $[\mathbf{1 3}]$ of the invariance of the group $\widehat{H F}(M)$ under isotopies of the Heegaard diagram for $M$, pseudo-holomorphic 3-gons were not used. Lipshitz observed in [8] that the induced chain map could be defined in terms of counting 3-gons.

Proof. We omit explicit analysis of isotopies which do not introduce or annihilate intersection points between $\alpha$ and $\beta$ arcs (i.e., preserve $\mathcal{Z}$ and $\mathcal{G}$ ); these just induce intersection-preserving isotopies on the Heegaard diagram. Below we will study two type of isotopies, which introduce new intersection points — we need not study isotopies, which remove intersection points, since filtered chain homotopy equivalence is an equivalence relation and is this symmetric.

First consider an isotopy of the type illustrated in Figure 16. This type of isotopy induces a pair of analogous isotopies of the Heegaard diagram, and one can define the obvious triangle injection. All components of associated domains appear far from the new intersection points and are of the form shown in Figure 13.

One could also perform an isotopy of a fork diagram near a puncture point, as shown in Figure 17. 
(a)

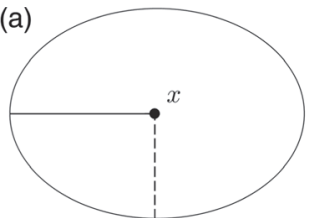

(b)

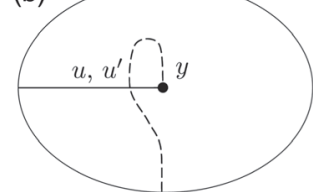

Figure 17. Introducing new intersections via an isotopy. (a) Before isotopy and (b) After isotopy.

(a)

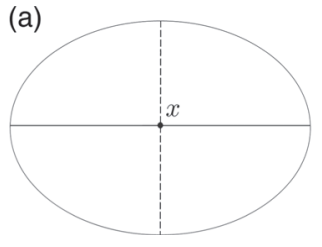

(b)

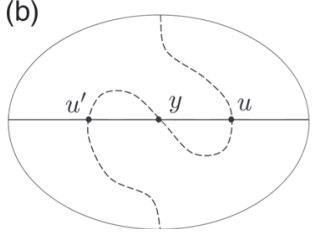

Figure 18. Isotopic Heegaard diagrams for $\Sigma(K) \#\left(S^{2} \times S^{1}\right)$ covering the fork diagrams in Figure 17. (a) Before isotopy and (b) After isotopy.

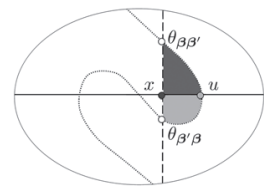

Figure 19. Local regions in domains of the 3-gons $\psi_{g_{\text {iso }}}^{+}($dark gray $)$and $\psi_{g_{\text {iso }}}^{-}$(light gray).

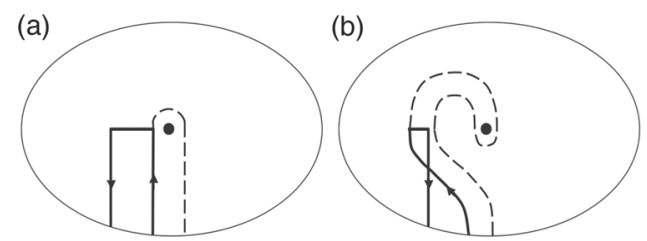

Figure 20. Loops used to compute gradings for $x(a)$ and $u(b)$.

Taking the two-fold cover of this local fork diagram branched on the one puncture gives the local Heegaard diagrams for $\Sigma(K) \#\left(S^{2} \times S^{1}\right)$ shown in Figure 18. The isotopy on the fork diagram amounts to an isotopy on the Heegaard diagram, and by Lemma 5.0.6 it is sufficient to construct a $\boldsymbol{\beta}$-triangle injection $g_{\text {iso }}$ and check that it preserves $R$.

We see from the 3 -sided region in Figure 19 that we can define the injection $g_{\text {iso }}$ such that $g_{\text {iso }}(x \mathbf{z})=u \mathbf{z}$. Furthermore, the loops in Figure 20 
show that $Q^{*}(u)=Q^{*}(x), P^{*}(u)=P^{*}(x)$, and $T(u \mathbf{z})=T(x \mathbf{z})$. Therefore, $R(u \mathbf{z})=R(x \mathbf{z})$.

\subsection{Invariance under choice of braid.}

Proof of Theorem 1.0.1. It suffices to verify that if $b$ and $b^{\prime}$ (inducing Heegaard diagrams $\mathcal{H}$ and $\mathcal{H}^{\prime}$ for $\left.\Sigma(K) \#\left(S^{2} \times S^{1}\right)\right)$ are related by a Birman move, then the $\rho$-filtered complexes $\widehat{C F}(\mathcal{H}, \mathfrak{s})$ and $\widehat{C F}\left(\mathcal{H}^{\prime}, \mathfrak{s}\right)$ have the same filtered chain homotopy type for each torsion $\mathfrak{s} \in \operatorname{Spin}^{c}\left(\Sigma(K) \#\left(S^{2} \times S^{1}\right)\right)$.

We first claim that we do not need to explicitly examine Birman moves of the form $b \mapsto g b$, where $b \in B_{2 n}$ and $g \in K_{2 n}$. Well, by Lemmas 5.0.10 and 5.0 .11 , the $R$-filtered complexes $\widehat{C F}_{*}\left(\mathcal{H}_{g b}, \mathfrak{s}\right)$ and $\widehat{C F}_{*}\left(\mathcal{H}_{b f}, \mathfrak{s}\right)$ are filtered chain isomorphic, where $f=-g^{-1}$. We will not explicitly analyze moves of the form $b \longmapsto b(-g)$ for generators $g$ of $K_{2 n}$, but the local pictures would be mirror images of those for $b \longmapsto b g$ and the arguments would be completely analogous.

We will see that each Birman move induces either a diffeomorphism of the Heegaard surface or a sequence of isotopies and handleslides relating Heegaard diagrams for $\Sigma(K) \#\left(S^{2} \times S^{1}\right)$ induced by the fork diagrams before and after the move (also preceded by a Heegaard stabilization in the Birman stabilization move).

For the case of a surface diffeomorphism, one obtains a chain complex isomorphism which will be shown to preserve $R$. By Lemma 5.0.8, such an isomorphism also preserves the filtration $\rho$.

We saw in Section 2.1 that isotopies and handleslides on Heegaard diagrams induce chain homotopy equivalences on $\widehat{C F}\left(\Sigma(K) \#\left(S^{2} \times S^{1}\right)\right)$ which count pseudo-holomorphic 3-gon classes of index zero. For each isotopy or handleslide taking $\boldsymbol{\alpha}$ and $\boldsymbol{\beta}$ to $\boldsymbol{\alpha}^{\prime}$ and $\boldsymbol{\beta}^{\prime}$, we will define a triangle injection $g: \mathbb{T}_{\boldsymbol{\alpha}} \cap \mathbb{T}_{\boldsymbol{\beta}} \hookrightarrow \mathbb{T}_{\boldsymbol{\alpha}^{\prime}} \cap \mathbb{T}_{\boldsymbol{\beta}^{\prime}}$. By Corollary 5.0.7, it suffices to construct these injections $g$ and verify that $R(g(\mathbf{x}))=R(\mathbf{x})$ for each $\mathbf{x} \in \mathcal{G}$. Note that since the moves induce local changes only, we will only demonstrate local regions in the domains of the 3-gons lying in neighborhoods of the moves. We exhibit such domains and check gradings in Section 5.3.

\subsection{Local effects of Birman moves.}

5.3.1. $\boldsymbol{b} \mapsto \boldsymbol{b} \boldsymbol{A}^{ \pm \mathbf{1}}=\boldsymbol{b} \boldsymbol{\sigma}_{\mathbf{1}}^{ \pm \mathbf{1}}$. The fork diagrams for $b \mapsto b A$ can be seen in Figure 21. This move induces a diffeomorphism on the Heegaard surface for $\Sigma(K) \#\left(S^{2} \times S^{1}\right)$ (in fact, a single Dehn twist) which sends $x_{i} \mapsto y_{i}$ for $i=1,2$ and sends $\left\{u, u^{\prime}\right\} \hookrightarrow\left\{v, v^{\prime}\right\}$. One thus obtains a chain isomorphism $g_{A}$ on Heegaard Floer complexes, and we verify that for each $\mathbf{w} \in \mathcal{G}$, $R\left(g_{A}(\mathbf{w})\right)=R(\mathbf{w})$. 
(a)

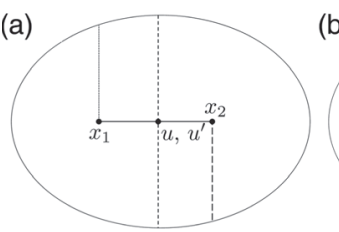

(b)

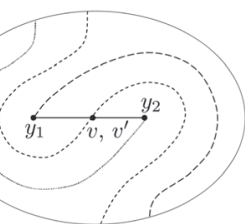

Figure 21. Fork diagrams associated to the move $b \mapsto b A$ (a) Local diagram for $b$ and (b) Local diagram for $b A$.

(a)

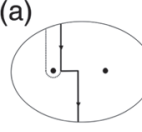

(b)

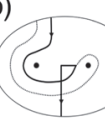

(c)

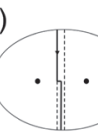

(d)

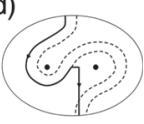

(e) (f)

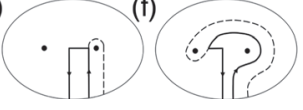

Figure 22. Loops associated to elements of $\mathcal{Z}$ affected by $b \mapsto b A$. (a) $x_{1}$, (b) $y_{2}$, (c) $u$, (d) $v$, (e) $x_{2}$ and (f) $y_{1}$.

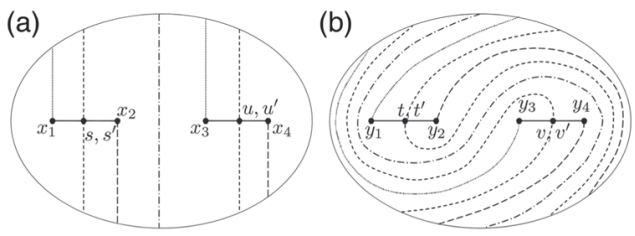

Figure 23. Fork diagrams associated to $b \mapsto b C_{i}$. (a) Local diagram for $b$ and (b) Local diagram for $b C_{i}$.

Since $R$ is stable, we only need to examine one $\mathcal{Z}$ representative for each element of $\widetilde{\mathcal{Z}} \backslash \tau$. By examining the local pictures in Figure 22, one can verify that $Q\left(g_{A}(\mathbf{w})\right)=Q(\mathbf{w})+1$ and $P\left(g_{A}(\mathbf{w})\right)=P(\mathbf{w})+1$ and all $\mathbf{w} \in \mathcal{G}$. Since moves are local and at most one component is modified, $T$ is preserved. The number of strands $n$ is also preserved, but $\epsilon$ and $w$ each increase by 1 . So, $s_{R}(b A)=s_{R}(b)$, and thus $R\left(g_{A}(\mathbf{w})\right)=R(\mathbf{w})$ for all $\mathbf{w} \in \mathcal{G}$. The details for the move $b \mapsto b A^{-1}$ are analogous.

5.3.2. $b \mapsto b C_{i}^{ \pm 1}=b\left(\sigma_{2 i} \sigma_{2 i-1} \sigma_{2 i+1} \sigma_{2 i}\right)^{ \pm 1}$. Figure 23 depicts local pictures of fork diagrams before and after this move. This move induces a sequence of two Dehn twists, mapping intersections via $x_{1} \mapsto y_{3}, x_{2} \mapsto y_{4}$, $x_{3} \mapsto y_{1}, x_{4} \mapsto y_{2},\left\{s, s^{\prime}\right\} \hookrightarrow\left\{v, v^{\prime}\right\}$, and $\left\{u, u^{\prime}\right\} \hookrightarrow\left\{t, t^{\prime}\right\}$. By examining the local pictures in Figure 24, one can verify that for each $\mathbf{w} \in \mathcal{G}, Q\left(g_{C_{i}}(\mathbf{w})\right)=$ $Q(\mathbf{w})+2, P\left(g_{C_{i}}(\mathbf{w})\right)=P(\mathbf{w})$, and $T\left(g_{C_{i}}(\mathbf{w})\right)=T(\mathbf{w})+1$. Here $n$ and $w$ are unchanged, but $\epsilon$ increases by 4 . So, $s_{R}\left(b C_{i}\right)=s_{R}(b)+1$, and thus $R\left(g_{C_{i}}(\mathbf{w})\right)=R(\mathbf{w})$ for all $\mathbf{w} \in \mathcal{G}$. The proof associated to the move $b \mapsto$ $b C_{i}^{-1}$ is analogous. 
(a)

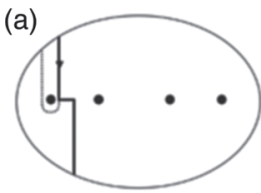

(b)

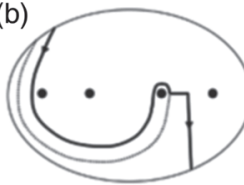

(e)

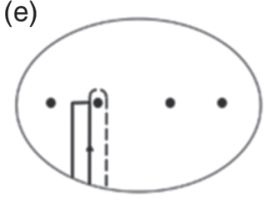

(f)

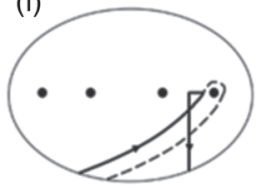

(i)

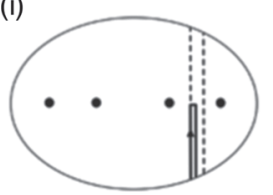

(j)

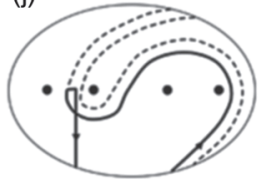

(c)

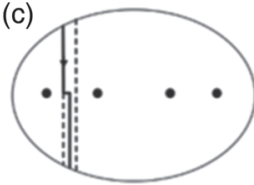

(g)

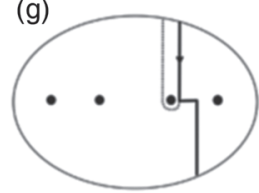

(k)

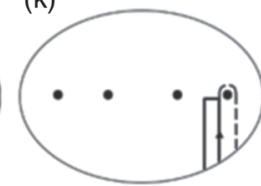

(d)

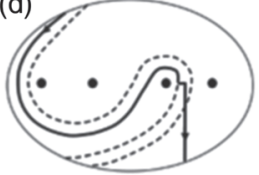

(h)

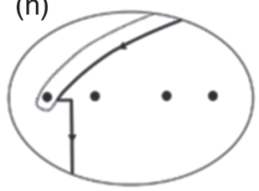

(I)

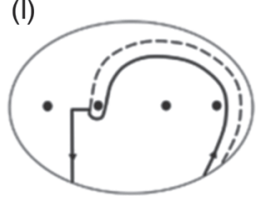

Figure 24. Loops associated to elements of $\mathcal{Z}$ affected by $b \mapsto b C_{i}$. (a) $x_{1}$, (b) $y_{3}$, (c) $s$, (d) $v$, (e) $x_{2}$, (f) $y_{4}$, (g) $x_{3}$, (h) $y_{1}$, (i) $u,(\mathrm{j}) t,(\mathrm{k}) x_{4}$ and (l) $y_{2}$.

5.3.3. $\boldsymbol{b} \mapsto b B^{ \pm 1}=\boldsymbol{b}\left(\sigma_{2} \sigma_{1}^{2} \sigma_{2}\right)^{ \pm 1}$. The fork diagrams before and after this move can be seen in Figure 25. To better understand the fork diagram for $b B$, we perform the isotopy resulting in Figure 25(c).

It is clear that only $\alpha_{2}$ is altered by the move. To be more precise, we take a look at the Heegaard diagrams for $\Sigma(K) \#\left(S^{2} \times S^{1}\right)$ which are the branched double-covers of the fork diagrams for $b$ and $b B$. These can be seen in Figure 26.

To get from the left diagram to the right, we can perform a sequence of two handleslides as shown in Figure 27.

We first address admissibility of the intermediate pointed Heegaard dia$\operatorname{gram}\left(\Sigma ; \boldsymbol{\alpha}^{2} ; \boldsymbol{\beta} ;+\infty\right)$.

Label the $m$ domains of $\left(\Sigma ; \boldsymbol{\alpha}^{1} ; \boldsymbol{\beta} ;+\infty\right)$ as indicated in Figure 29(a), where $\mathcal{D}_{k}$ lies entirely outside of the picture for $k \geq 11$; label the $(m+4)$ domains of $\left(\Sigma ; \boldsymbol{\alpha}^{2} ; \boldsymbol{\beta} ;+\infty\right)$ as indicated $29(\mathrm{~b})$, where $\mathcal{D}_{k}^{\prime}$ corresponds to $\mathcal{D}_{k}$ for $k \geq 11$. Now consider some periodic domain in $\left(\Sigma ; \boldsymbol{\alpha}^{2} ; \boldsymbol{\beta} ;+\infty\right)$

$$
\mathcal{P}^{\prime}=\widetilde{c}_{1} \widetilde{\mathcal{D}}_{1}+\widetilde{c}_{2} \widetilde{\mathcal{D}}_{2}+\widetilde{c}_{1} \widetilde{\mathcal{D}}_{3}+\widetilde{c}_{1} \widetilde{\mathcal{D}}_{4}+\sum_{i=1}^{m} c_{i} \mathcal{D}_{i}^{\prime} .
$$

Note that

$$
\begin{aligned}
c_{1}-c_{10} & =\widetilde{c}_{1}-c_{10}=c_{5}-c_{9}=\widetilde{c}_{i}-c_{i} \quad \text { for } \quad 1 \leq i \leq 4 \quad \text { and } \\
\widetilde{c}_{2}-c_{9} & =\widetilde{c}_{3}-c_{8}=\widetilde{c}_{4}-c_{7}=c_{10}-c_{6} .
\end{aligned}
$$



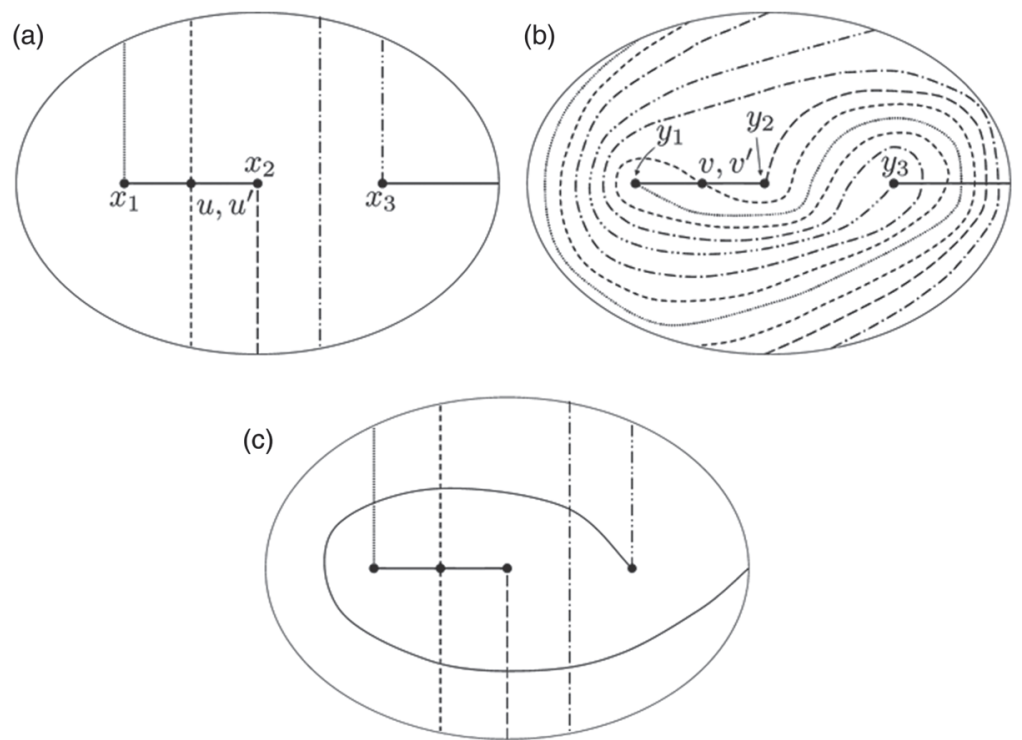

Figure 25. Fork diagrams associated to $b \mapsto b B$. (a) Local diagram for $b$, (b) Local diagram for $b B$ and (c) Isotopic to Figure 25(b).
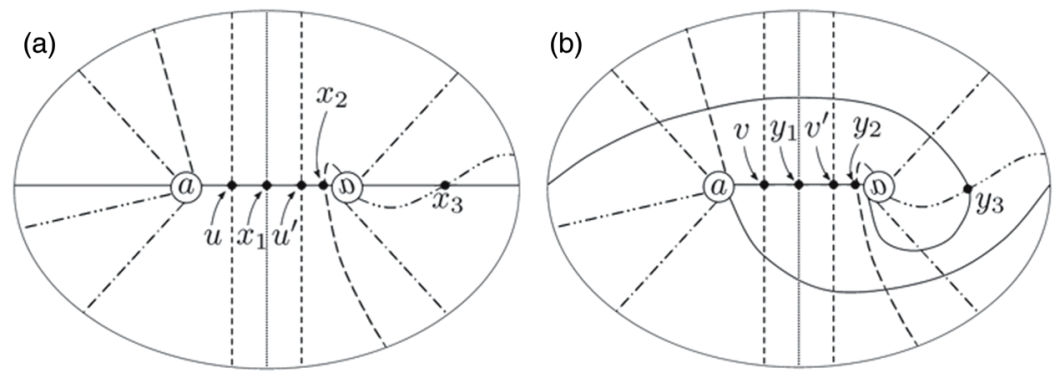

Figure 26. Heegaard diagrams for $\Sigma(K) \#\left(S^{2} \times S^{1}\right)$ covering the fork diagrams in Figure 25. (a) From $b$ and (b) From $b B$.

As a result,

$$
\widetilde{c}_{1}=c_{1} \quad \text { and } \quad c_{2}-c_{9}=c_{3}-c_{8}=c_{4}-c_{7}=c_{1}-c_{6} .
$$

So, we can conclude that there is a periodic domain in $\left(\Sigma ; \boldsymbol{\alpha}^{1} ; \boldsymbol{\beta} ;+\infty\right)$ of the form

$$
\mathcal{P}=\sum_{i=1}^{m} c_{i} \mathcal{D}_{i}
$$

But since $\left(\Sigma ; \boldsymbol{\alpha}^{1} ; \boldsymbol{\beta} ;+\infty\right)$ is admissible, there are both positive and negative integers among the $c_{i}$, and thus $\left(\Sigma ; \boldsymbol{\alpha}^{2} ; \boldsymbol{\beta} ;+\infty\right)$ is also admissible. 
(a)

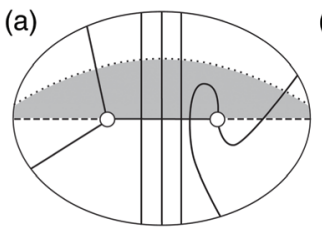

(b)

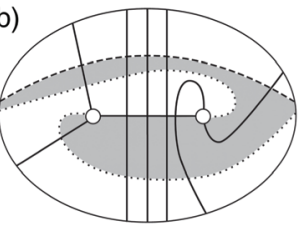

Figure 27. Two handleslides connecting Heegaard diagrams for $b$ and $b B$. In each picture, the pair of pants is shaded, the new circle is dotted, and the old one is dashed. (a) $\boldsymbol{\alpha}=\boldsymbol{\alpha}^{1} \mapsto$ $\boldsymbol{\alpha}^{2}$ and (b) $\boldsymbol{\alpha}^{2} \mapsto \boldsymbol{\alpha}^{3}=\boldsymbol{\alpha}^{\prime}$.

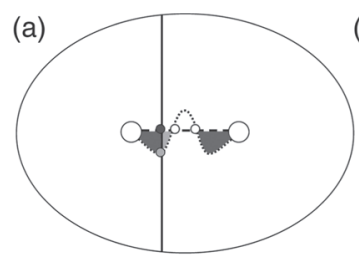

(b)

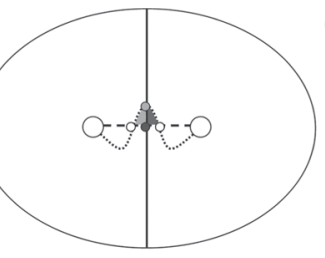

(c)

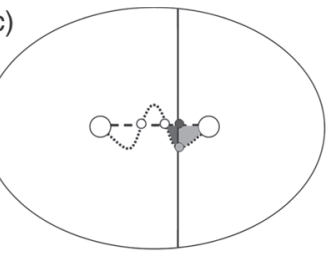

(d)

(e)
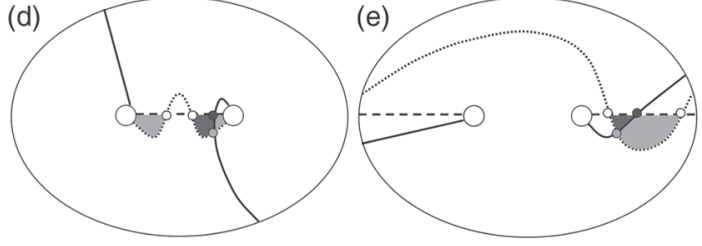

Figure 28. Local regions in domains of 3 -gons $\psi^{+}$(dark gray) and $\psi^{-}$(light gray) for $g_{B}^{a}$. White dots are components of $\boldsymbol{\theta}_{\boldsymbol{\alpha}^{\prime \prime} \boldsymbol{\alpha}}, \boldsymbol{\theta}_{\boldsymbol{\alpha} \boldsymbol{\alpha}^{\prime}} \in \mathbb{T}_{\boldsymbol{\alpha}} \cap \mathbb{T}_{\boldsymbol{\alpha}^{\prime \prime}}$. (a) $u \mapsto w$, (b) $x_{1} \mapsto s_{1}$, (c) $u^{\prime} \mapsto w^{\prime}$, (d) $x_{2} \mapsto s_{2}$, (e) $x_{3} \mapsto s_{3}$.
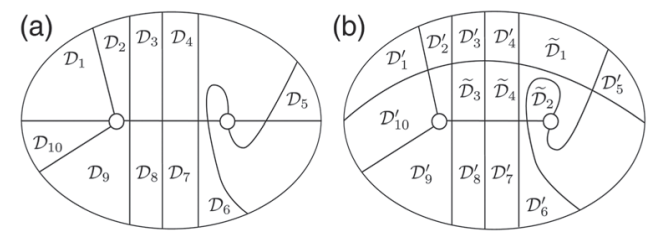

Figure 29. Domains of on Heegaard surfaces before and after the handleslide $\boldsymbol{\alpha}^{1} \rightarrow \boldsymbol{\alpha}^{2}$ induced by the Birman move $b \mapsto b B$. (a) $\Sigma \backslash\left(\cup_{i} \alpha_{i}^{1}\right) \backslash\left(\cup_{i} \beta_{i}\right)$ and (b) $\Sigma \backslash\left(\cup_{i} \alpha_{i}^{2}\right) \backslash\left(\cup_{i} \beta_{i}\right)$.

Let the injection $g_{B}$ act as $x_{1} x_{3} \mathbf{y} \mapsto y_{1} y_{3} \mathbf{y}, x_{2} x_{3} \mathbf{y} \mapsto y_{2} y_{3} \mathbf{y}, u x_{3} \mathbf{y} \mapsto v y_{3} \mathbf{y}$, $u^{\prime} x_{3} \mathbf{y} \mapsto v^{\prime} y_{3} \mathbf{y}, x_{1} \mathbf{z} \mapsto y_{1} \mathbf{z}, x_{2} \mathbf{z} \mapsto y_{2} \mathbf{z}, u \mathbf{z} \mapsto v \mathbf{z}$, and $u^{\prime} \mathbf{z} \mapsto v^{\prime} \mathbf{z}$. Note that $\mathbf{z}$ is an $(n-1)$-tuple whose component on the $\alpha_{2}$ arc is not shown in the local fork diagram. 

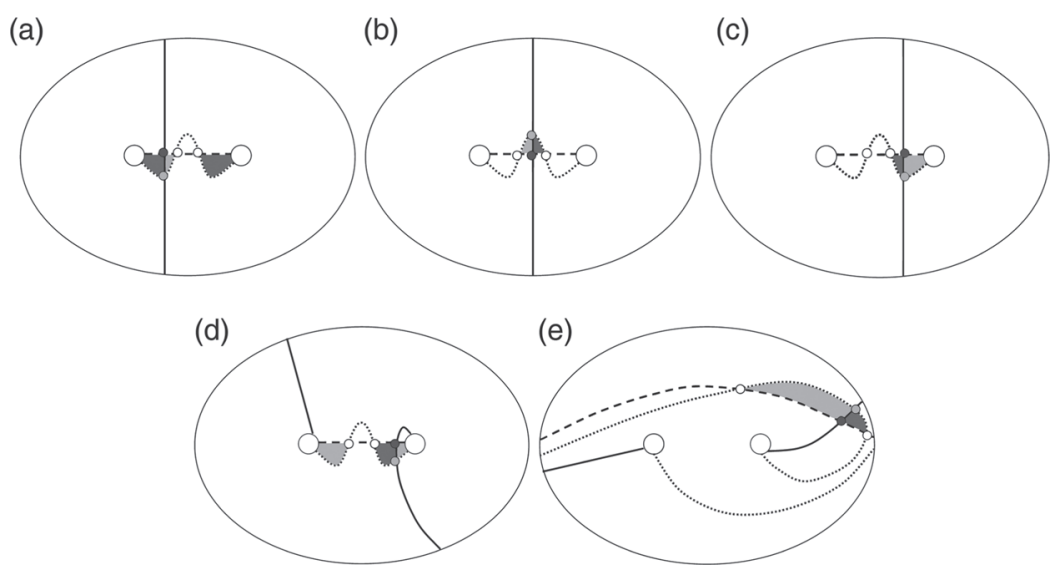

Figure 30. Local regions in domains of 3 -gons $\psi^{+}$(dark gray) and $\psi^{-}$(light gray) for $g_{B}^{b}$. White dots are components of $\boldsymbol{\theta}_{\boldsymbol{\alpha}^{\prime} \boldsymbol{\alpha}^{\prime \prime}}, \boldsymbol{\theta}_{\boldsymbol{\alpha}^{\prime \prime} \boldsymbol{\alpha}^{\prime}} \in \mathbb{T}_{\boldsymbol{\alpha}^{\prime \prime}} \cap \mathbb{T}_{\boldsymbol{\alpha}^{\prime}}$. (a) $w \mapsto v$, (b) $s_{1} \mapsto y_{1}$, (c) $w^{\prime} \mapsto v^{\prime}$, (d) $s_{2} \mapsto y_{2}$ and (e) $s_{3} \mapsto y_{3}$.

(a)

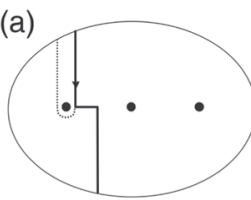

(e)

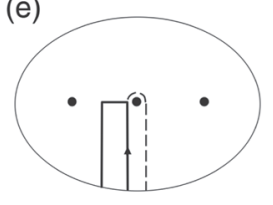

(b)

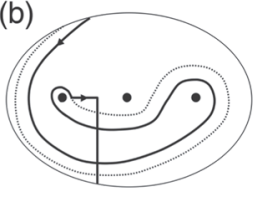

(c)

(f)

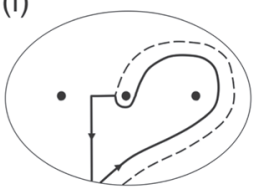

(g)

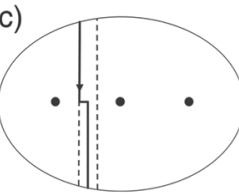

(d)

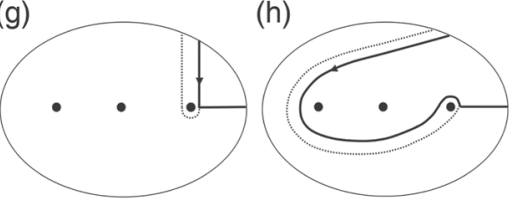

Figure 31. Loops associated to elements of $\mathcal{Z}$ affected by $b \mapsto b B$. (a) $x_{1}$, (b) $y_{1}$, (c) $u$, (d) $v$, (e) $x_{2}$, (f) $y_{2}$, (g) $x_{3}$ and (h) $y_{3}$.

When viewed as a function on $\mathbb{T}_{\boldsymbol{\alpha}} \cap \mathbb{T}_{\boldsymbol{\beta}}, g_{B}$ is a composition $g_{B}^{\boldsymbol{\alpha}, 2} \circ g_{B}^{\boldsymbol{\alpha}, 1}$ of triangle injections corresponding to the two handleslides. Local regions in domains of 3 -gons for $g_{B}^{\boldsymbol{\alpha}, 1}$ (resp. $g_{B}^{\boldsymbol{\alpha}, 2}$ ) can be seen in Figure 28 (resp. 30). One can verify that if two of these regions appear in the domain of a 3 -gon associated with $g_{B}^{\boldsymbol{\alpha}, 1}$, then there are neighborhoods of these regions which map to disjoint neighborhoods in the fork diagram downstairs (and likewise for those associated with $g_{B}^{\boldsymbol{\alpha}, 2}$ ). As a result, all 3-gons presented here avoid the anti-diagonal $\nabla$. 
(a)

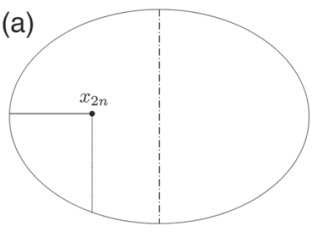

(b)

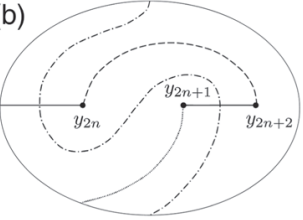

Figure 32. Fork diagrams associated to $b \in B_{2 n} \mapsto b \sigma_{2 n} \in$ $B_{2 n+2}$. (a) Local diagram for $b \in B_{2 n}$ and (b) Local diagram for $b \sigma_{2 n} \in B_{2 n+2}$. (a) Local diagram for $b \in B_{2 n}$ and (b) Local diagram for $b \sigma_{2 n} \in B_{2 n+2}$.
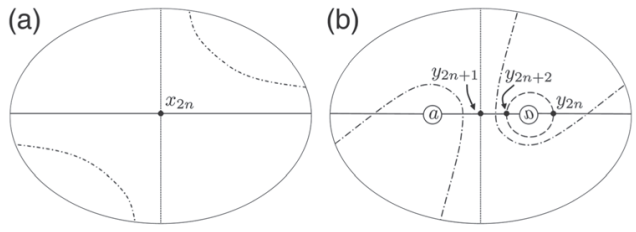

Figure 33. Heegaard diagrams for $\Sigma(K) \#\left(S^{2} \times S^{1}\right)$ covering the fork diagrams in Figure 32. (a) From $b \in B_{2 n}$, (b) From $b \sigma_{2 n} \in B_{2 n+2}$.

We see from Figure 31 that $Q\left(y_{1} y_{3} \mathbf{y}\right)=Q\left(x_{1} x_{3} \mathbf{y}\right)+3, Q\left(v y_{3} \mathbf{y}\right)=$ $Q\left(u x_{3} \mathbf{y}\right)+3, Q\left(y_{1} \mathbf{z}\right)=Q\left(x_{1} \mathbf{z}\right)+1, Q\left(y_{2} y_{3} \mathbf{y}\right)=Q\left(x_{2} x_{3} \mathbf{y}\right)+3, Q\left(y_{2} \mathbf{z}\right)=$ $Q\left(x_{2} \mathbf{z}\right)+1, Q(v \mathbf{z})=Q(u \mathbf{z})+1$, and $P\left(g_{B}(\mathbf{w})\right)=P(\mathbf{w})$ for all $\mathbf{w} \in \mathcal{G}$.

Furthermore, one can check that $T\left(y_{1} y_{3} \mathbf{y}\right)=T\left(x_{1} x_{3} \mathbf{y}\right)+2, T\left(v y_{3} \mathbf{y}\right)=$ $T\left(u x_{3} \mathbf{y}\right)+2, T\left(y_{1} \mathbf{z}\right)=T\left(x_{1} \mathbf{z}\right), T\left(y_{2} y_{3} \mathbf{y}\right)=T\left(x_{2} x_{3} \mathbf{y}\right)+2, T\left(y_{2} \mathbf{z}\right)=T\left(x_{2} \mathbf{z}\right)$, and $T(v \mathbf{z})=T(u \mathbf{z})$. This move preserves $n$ and $w$, but increases $\epsilon$ by 4 . Thus $s_{R}(b B)=s_{R}(b)+1$ and $R\left(g_{B}(\mathbf{w})\right)=R(\mathbf{w})$ for all $\mathbf{w} \in \mathcal{G}$. The proof associated to the move $b \mapsto b B^{-1}$ is analogous.

5.3.4. $b \in \boldsymbol{B}_{\mathbf{2} n} \leftrightarrow b \boldsymbol{\sigma}_{\mathbf{2} n} \in \boldsymbol{B}_{\mathbf{2 n + 2}}$. Fork diagrams before and after stabilization can be seem in Figure 32, and the induced Heegaard diagrams in Figure 33.

The stabilization braid move corresponds to a Heegaard diagram stabilization followed by several handleslides, which can be seen in Figure 34. The destabilization braid move induces the inverse of this sequence of Heegaard moves.

We first address admissibility. Clearly stabilizing or destabilizing an admissible Heegaard diagram yields another admissible one. By analyzing domains, it is not hard to verify that the handleslides in Figure 34 preserve admissibility; we will demonstrate this explicitly for third handleslide $\left(\boldsymbol{\beta}^{2} \mapsto \boldsymbol{\beta}^{3}\right)$, and leave the rest as an exercise to the reader. 
(a)

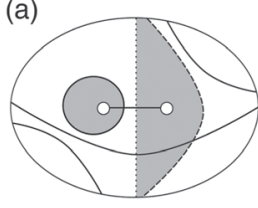

(b)

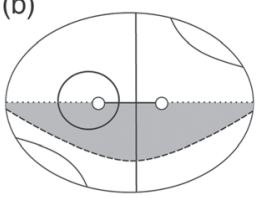

(c)

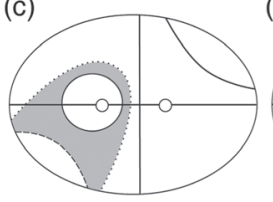

(d)

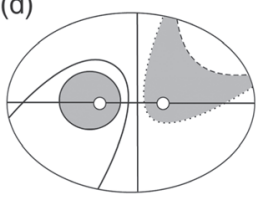

Figure 34. Four handleslides connecting Heegaard diagrams for $b$ and $b \sigma_{2 n}$. In each picture, the pair of pants is shaded, the new circle is dotted, and the old one is dashed. (a) $\boldsymbol{\beta}=\boldsymbol{\beta}^{1} \mapsto \boldsymbol{\beta}^{2}$, (b) $\boldsymbol{\alpha} \mapsto \boldsymbol{\alpha}^{\prime}$, (c) $\boldsymbol{\beta}^{2} \mapsto \boldsymbol{\beta}^{3}$, and (d) $\boldsymbol{\beta}^{3} \mapsto \boldsymbol{\beta}^{4}=\boldsymbol{\beta}^{\prime}$.

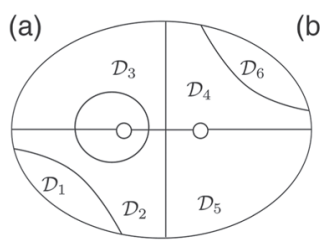

(b) $\tilde{\tilde{D}}_{1}$

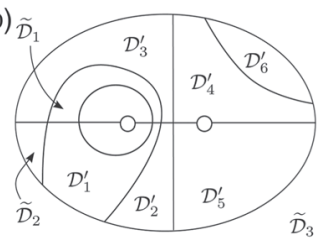

Figure 35. Domains on Heegaard surfaces before and after the handleslide $\boldsymbol{\beta}^{2} \mapsto \boldsymbol{\beta}^{3}$ induced by Birman stabilization. (a) $\Sigma \backslash\left(\cup_{i} \alpha_{i}\right) \backslash\left(\cup_{i} \beta_{i}^{2}\right)$ and (b) $\Sigma \backslash\left(\cup_{i} \alpha_{i}\right) \backslash\left(\cup_{i} \beta_{i}^{3}\right)$.

Assume that $\left(\Sigma ; \boldsymbol{\alpha} ; \boldsymbol{\beta}^{2} ;+\infty\right)$ is admissible. Label the $m$ domains of $\left(\Sigma ; \boldsymbol{\alpha} ; \boldsymbol{\beta}^{2} ;+\infty\right)$ as indicated in Figure $35(\mathrm{a})$, where $\mathcal{D}_{k}$ lies entirely outside of the picture for $k \geq 7$; label the $(m+2)$ domains of $\left(\Sigma ; \boldsymbol{\alpha} ; \boldsymbol{\beta}^{3} ;+\infty\right)$ as indicated in Figure 35(b), where $\mathcal{D}_{k}^{\prime}$ corresponds to $\mathcal{D}_{k}$ for $k \geq 7$. Now consider some periodic domain in $\left(\Sigma ; \boldsymbol{\alpha} ; \boldsymbol{\beta}^{3} ;+\infty\right)$

$$
\mathcal{P}^{\prime}=\widetilde{c}_{1} \widetilde{\mathcal{D}}_{1}+\widetilde{c}_{2} \widetilde{\mathcal{D}}_{2}+\sum_{i=1}^{m} c_{i} \mathcal{D}_{i}^{\prime}
$$

Now because $\mathcal{P}^{\prime}$ is periodic, we have that

$$
\widetilde{c}_{2}-c_{1}=c_{2}-c_{1}, \quad \text { and so } \quad \widetilde{c}_{2}=c_{2} .
$$

Therefore, there is a periodic domain in $\left(\Sigma ; \boldsymbol{\alpha} ; \boldsymbol{\beta}^{2} ;+\infty\right)$ given by

$$
\mathcal{P}=\sum_{i=1}^{m} c_{i} \mathcal{D}_{i}
$$

Because $\left(\Sigma ; \boldsymbol{\alpha} ; \boldsymbol{\beta}^{2} ;+\infty\right)$ is admissible, there is at least one positive $c_{i}$ and at least one negative $c_{i}$. So, $\mathcal{P}^{\prime}$ has positive and negative coefficients, and thus $\left(\Sigma ; \boldsymbol{\alpha} ; \boldsymbol{\beta}^{3} ;+\infty\right)$ is also admissible.

Let $x_{2 n+1}$ denote the additional intersection obtained via Heegaard stabilization. We define the injection $g_{s t a b}$ as $x_{2 n} x_{2 n+1} \mathbf{v} \mapsto y_{2 n+1} y_{2 n} \mathbf{v}$ and $x_{2 n+1} \mathbf{z} \mapsto y_{2 n+2} \mathbf{z}$, where $\mathbf{v}$ is an $(n-1)$-tuple not contained in the local 
(a)

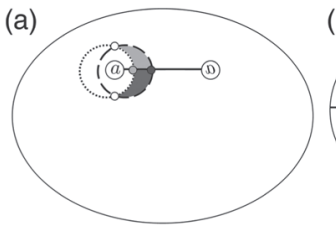

(b)

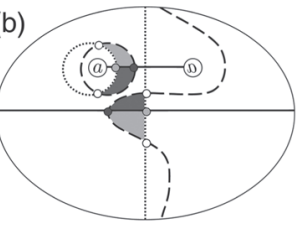

Figure 36. Local regions in domains of the 3 -gons $\psi^{+}$(dark gray) and $\psi^{-}$(light gray) for $g_{\text {stab }}^{\boldsymbol{\beta}, 1}$. White dots are components of $\boldsymbol{\theta}_{\boldsymbol{\beta} \boldsymbol{\beta}^{2}}, \boldsymbol{\theta}_{\boldsymbol{\beta}^{2} \boldsymbol{\beta}} \in \mathbb{T}_{\boldsymbol{\beta}} \cap \mathbb{T}_{\boldsymbol{\beta}^{2}}$. (a) $x_{2 n+1} \mapsto w_{2 n+1}$, and (b) $x_{2 n} x_{2 n+1} \mapsto w_{2 n} w_{2 n+1}$.

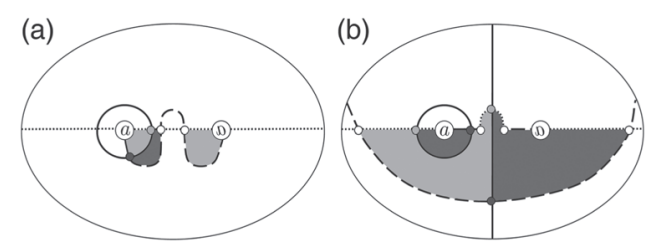

Figure 37. Local regions in domains of the 3-gons $\psi^{+}$(dark gray) and $\psi^{-}$(light gray) for $g_{s t a b}^{\alpha}$. White dots are components of $\boldsymbol{\theta}_{\boldsymbol{\alpha} \boldsymbol{\alpha}^{\prime}}, \boldsymbol{\theta}_{\boldsymbol{\alpha}^{\prime} \boldsymbol{\alpha}} \in \mathbb{T}_{\boldsymbol{\alpha}} \cap \mathbb{T}_{\boldsymbol{\alpha}^{\prime}}$. (a) $w_{2 n+1} \mapsto y_{2 n+2}$ and (b) $w_{2 n} w_{2 n+1} \mapsto y_{2 n+1} y_{2 n}$.

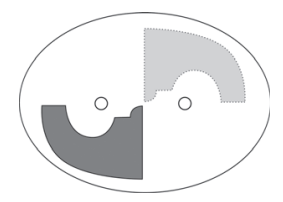

Figure 38. Arranging that a 3-gon avoids the anti-diagonal. The dark gray region is the domain $\mathcal{D}$, and the light gray region is the other connected component of $\pi\left(\pi^{-1}(\mathcal{D})\right)$.

picture, and $\mathbf{z}$ is an $n$-tuple not contained within the local picture. In fact, $g_{\text {stab }}$ can be written as a composition $g_{\text {stab }}^{\boldsymbol{\beta}, 3} \circ g_{\text {stab }}^{\boldsymbol{\beta}, 2} \circ g_{\text {stab }}^{\boldsymbol{\alpha}} \circ g_{\text {stab }}^{\boldsymbol{\beta}, 1}$ of triangle injections corresponding to the four handleslides in Figure 34 . All of the 3 -gons required for $g_{\text {stab }}^{\boldsymbol{\beta}, 2}$ and $g_{\text {stab }}^{\boldsymbol{\beta}, 3}$ are 3 -gons with "small" domains, and we will not exhibit these in figures. Local regions in domains of 3 -gons for $g_{\text {stab }}^{\boldsymbol{\beta}, 1}$ and $g_{\text {stab }}^{\boldsymbol{\alpha}}$ are exhibited in Figures 36 and 37, respectively. Note that the 3 -gon appearing in Figure 37(b) is of the second type (cf. Figure 1).

It can be arranged that these 3 -gons avoid the anti-diagonal $\nabla$ (making use of the discussion in Section 4.5.2). In particular, if $\mathcal{D}$ denotes one of the six-sided regions appearing in Figure 37(b) and $\pi: \Sigma \rightarrow S^{2}$ is the

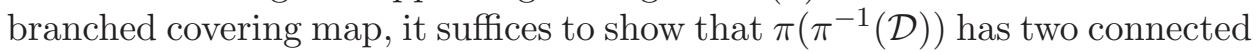
components. This is illustrated in Figure 38. 


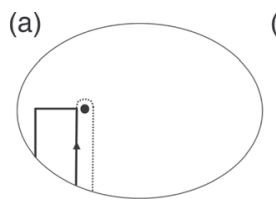

(b)

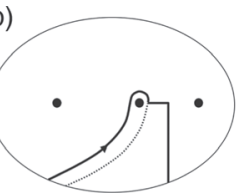

(c)

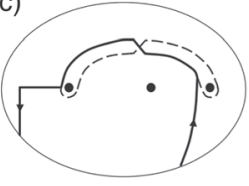

(d)

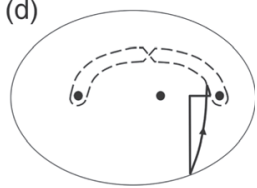

Figure 39. Loops for elements of $\mathcal{Z}$ affected by $b \in B_{2 n} \leftrightarrow$ $b \sigma_{2 n} \in B_{2 n+2}$. (a) $x_{2 n}$, (b) $y_{2 n+1}$ and (c) $y_{2 n}$ and (d) $y_{2 n+2}$.

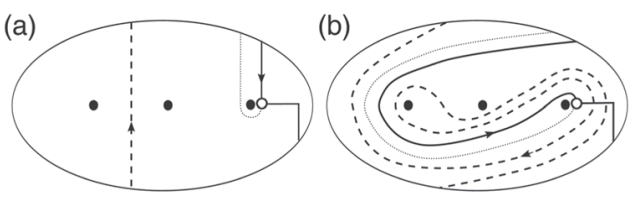

Figure 40. The effect of the move $b \mapsto b B$ on a pass-through arc appearing in a grading loop. (a) Grading loop for $u \mathbf{x}$, (b) Grading loop for $u^{\prime} \mathbf{x}$.

By examining the local pictures in Figure 39, one can verify that $Q\left(y_{2 n+1} y_{2 n} \mathbf{v}\right)=Q\left(x_{2 n} \mathbf{v}\right)+1, Q\left(y_{2 n+2} \mathbf{z}\right)=Q(\mathbf{z}), P\left(y_{2 n+1} y_{2 n} \mathbf{v}\right)=P\left(x_{2 n} \mathbf{v}\right)$, $P\left(y_{2 n+2} \mathbf{z}\right)=P(\mathbf{z}), T\left(y_{2 n+1} y_{2 n} \mathbf{v}\right)=T\left(x_{2 n} \mathbf{v}\right)+1$, and $T\left(y_{2 n+2} \mathbf{z}\right)=T(\mathbf{z})$. Stabilization adds two strands, increases $\epsilon$ by 1 , and decreases $w$ by 1 . Thus $s_{R}\left(b \sigma_{2 n}\right)=s_{R}(b)$ and so $R\left(y_{2 n+1} y_{2 n} \mathbf{v}\right)=R\left(x_{2 n} \mathbf{v}\right)$ and $R\left(y_{2 n+2} \mathbf{z}\right)=R(\mathbf{z})$.

We need not explicitly analyze the destabilization move at all, since filtered chain homotopy equivalence is an equivalence relation.

5.4. Generality of local pictures. It remains to justify that our local pictures above were sufficiently general:

(i) There could be some $u \in \widetilde{\mathcal{Z}}$ such that $u \mathbf{x} \in \mathcal{Z}$ and one of the loops used to compute gradings for $u \mathbf{x}$ contain vertical arcs that pass through the local diagram, as seen in Figure 40. One can verify that the grading contributions of such arcs are preserved by Birman moves.

(ii) Several $\beta$ arcs intersecting the interior of the same $\alpha$ arc can be isotoped to be very close to one another and thus behave identically under moves.

(iii) We assumed above that all $\beta$ arcs shown belong to distinct $\beta_{i}$. If two $\beta$ arcs share the same $\beta_{i}$, then fewer Bigelow generators are allowed.

(iv) The local pictures in Section 5.2 never have handles passing through them. A handle $b h_{i}$ contributes a vertical arc as in (5.4) to each loop associated to a point on $\beta_{i}$.

(v) For arcs terminating at punctures near the boundary of the local picture, we can modify the entrance trajectory (i.e., from above or from below) by applying an isotopy to the $\beta$ arc and shrinking the scope of the picture (see Figure 41). 
(a)

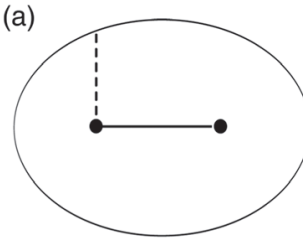

(b)

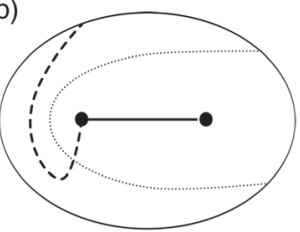

Figure 41. Modifying the entry trajectory of a $\beta$ arc terminating near the boundary. (a) Dashed arc enters from above and (b) Now from below.
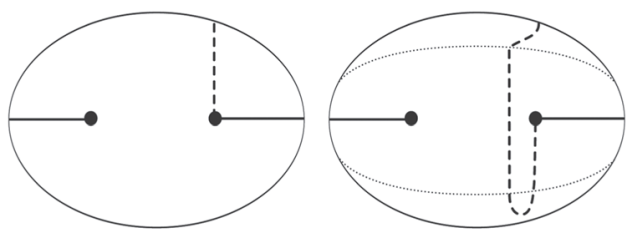

Figure 42. Modifying the entry trajectory of a $\beta$ arc terminating far from the boundary. (a) Dashed arc enters from above and (b) Now from below.

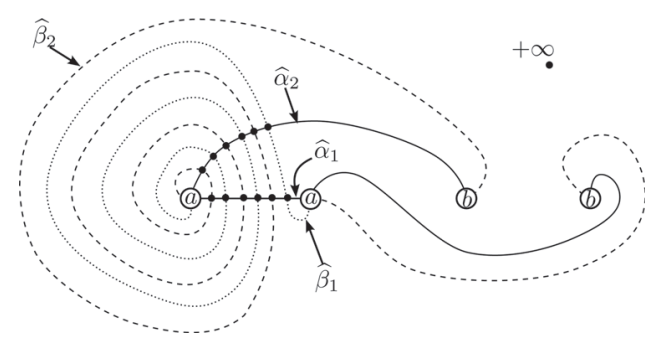

Figure 43. The Heegaard diagram for $L(3,1) \#\left(S^{1} \times S^{2}\right)$ obtained from $\sigma_{2}^{3} \in B_{4}$.

Entrance trajectories of $\beta$ arcs terminating far from the boundary can be modified in the same way, but at the expense of adding a passthrough arc to the local picture (see Figure 42).

\section{The left-handed trefoil and the lens space $L(3,1)$}

The fork diagram for the left-handed trefoil obtained from $\sigma_{2}^{3} \in B_{4}$ induces the admissible Heegaard diagram $\left(\Sigma_{2},\left\{\widehat{\alpha}_{1}, \widehat{\alpha}_{2}\right\},\left\{\widehat{\beta}_{1}, \widehat{\beta}_{2}\right\},+\infty\right)$ for $L(3,1) \#\left(S^{1} \times S^{2}\right)$ shown in Figure 43. Label the intersections on $\widehat{\alpha}_{1}$ from left to right as $s^{\prime}, t^{\prime}, x_{2}, t, s$, and $x_{1}$, and label those on $\widehat{\alpha}_{2}$ from bottom top as $x_{4}, v^{\prime}, u^{\prime}, x_{3}, u$, and $v$. 
Let us perform the calculation with $\mathbb{Z} / 2 \mathbb{Z}$ coefficients; this can be done combinatorially, since the diagram in Figure 43 is nice in the sense of [19].

The differential is thus

$$
\begin{gathered}
\widehat{\partial}\left(x_{2} x_{3}\right)=u t^{\prime}+u^{\prime} t, \quad \widehat{\partial}(u t)=s x_{3}+v x_{2}, \quad \widehat{\partial}\left(u t^{\prime}\right)=\widehat{\partial}\left(u^{\prime} t\right)=s v^{\prime}+s^{\prime} v, \\
\widehat{\partial}\left(u^{\prime} t^{\prime}\right)=s^{\prime} x_{3}+v^{\prime} x_{2}, \quad \widehat{\partial}\left(s^{\prime} v^{\prime}\right)=\widehat{\partial}\left(s x_{3}\right)=\widehat{\partial}\left(v x_{2}\right)=u^{\prime} x_{1}+t^{\prime} x_{4}, \\
\widehat{\partial}\left(s^{\prime} v\right)=\widehat{\partial}\left(s v^{\prime}\right)=x_{1} x_{4}+x_{1} x_{4}=0, \quad \widehat{\partial}\left(v^{\prime} x_{2}\right)=\widehat{\partial}\left(s^{\prime} x_{3}\right)=\widehat{\partial}(s v)=t x_{4}+u x_{1}, \\
\text { and } \widehat{\partial}\left(u^{\prime} x_{1}\right)=\widehat{\partial}\left(t^{\prime} x_{4}\right)=\widehat{\partial}\left(x_{1} x_{4}\right)=\widehat{\partial}\left(t x_{4}\right)=\widehat{\partial}\left(u x_{1}\right)=0 .
\end{gathered}
$$

Now recall that $L(3,1)$, has three $\operatorname{Spin}^{c}$ structures $\mathfrak{s}_{i}, i=0,1,2$. These induce three $\operatorname{Spin}^{c}$ structures on $L(3,1) \#\left(S^{1} \times S^{2}\right)$ given by $\mathfrak{s}_{\mathfrak{i}} \# \mathfrak{s}$, where $\mathfrak{s}$ is the unique torsion $\mathrm{Spin}^{c}$-structure on $S^{2} \times S^{1}$. One should observe that the diagram in Figure 43 can be related via handleslides to one which is the disjoint union of a diagram for $\Sigma(K)$ and the usual admissible genusone diagram for $S^{2} \times S^{1}$. As a result, all generators $\mathbf{x}$ in Figure 43 have $\mathfrak{s}_{+\infty}(\mathbf{x})=\mathfrak{s}_{\mathfrak{i}} \# \mathfrak{s}$. They partition this set of generators as

$$
\begin{aligned}
& \mathfrak{U}_{0}=\left\{x_{2} x_{3}, u t^{\prime}, u^{\prime} t, s^{\prime} v, s v^{\prime}, x_{1} x_{4}\right\}, \\
& \mathfrak{U}_{1}=\left\{u t, s^{\prime} v^{\prime}, s x_{3}, v x_{2} \cdot u^{\prime} x_{1}, t^{\prime} x_{4}\right\}, \\
& \mathfrak{U}_{2}=\left\{u^{\prime} t^{\prime}, v^{\prime} x_{2}, s^{\prime} x_{3}, s v, t x_{4}, u x_{1}\right\} .
\end{aligned}
$$

Note that the differential always lowers the $R$-grading by 1 in this case, and thus the left-handed trefoil is evidently $\rho$-degenerate. The $R$-grading then provides an absolute Maslov grading on the group $\widehat{H F}\left(L(3,1) \#\left(S^{1} \times\right.\right.$ $\left.\left.S^{2}\right) ; \mathbb{Z} / 2 \mathbb{Z}\right)$.

One can see that homology group decomposes with respect to the $R$-grading as

$$
\widehat{H F}\left(L(3,1) \#\left(S^{1} \times S^{2}\right) ; \mathbb{Z} / 2 \mathbb{Z}\right)=\left[(\mathbb{Z} / 2 \mathbb{Z})^{\oplus 3}\right]_{R=3 / 2} \oplus\left[(\mathbb{Z} / 2 \mathbb{Z})^{\oplus 3}\right]_{R=1 / 2} .
$$

\section{Reduced theory}

The sequel to the present paper [24] outlines a reduced theory which provides a filtration on the Heegaard Floer chain complex for $\Sigma(K)$. The reduced version is easier to compute, and it is shown in $[\mathbf{2 4}]$ that the spectral sequence discussed in the present paper is completely determined by an analogous reduced spectral sequence. The reduced theory can be shown to have some nice formal properties with respect to taking connected sums and mirrors of knots, and can be used to show that all two-bridge knots are $\rho$-degenerate. It would be tempting to speculate that all alternating knots are $\rho$-degenerate, but this is not known. 


\section{Future directions}

8.1. Relationship with $\boldsymbol{K} \boldsymbol{h}_{\text {symp, inv }}$. Given a pointed Heegaard diagram $\mathcal{H}$ for $\Sigma(K) \#\left(S^{2} \times S^{1}\right)$ coming from a braid, we saw that the filtration $\rho$ can only be defined on generators in torsion $\operatorname{Spin}^{c}$ structures. It would be interesting to investigate whether Heegaard diagrams encountered in this context actually contain generators in non-torsion Spin ${ }^{c}$ structures; if not, then $E^{1}$ is in fact all of $K h_{\text {symp, inv }}(K)$. In particular, we would obtain that when $K$ is $\rho$-degenerate,

$$
\widehat{H F}\left(\Sigma(K) \#\left(S^{2} \times S^{1}\right)\right) \cong K h_{\text {symp,inv }}(K)
$$

Since all $\operatorname{Spin}^{c}$ structures of $\Sigma(K)$ are torsion, one can define a reduced filtration $\rho$ on the entire complex $\widehat{C F}(\Sigma(K))$ - this is done in [24]. The $E^{1}$ page of the induced reduced spectral sequence is a reasonable candidate for a reduced version of $K h_{\text {symp,inv }}(K)$.

8.2. The Khovanov-Heegard Floer spectral sequence. Ozsváth and Szabó showed in [14] that the groups $\widehat{H F}(\Sigma(L)), \widehat{H F}\left(\Sigma\left(L_{0}\right)\right)$, and $\widehat{H F}$ $\left(\Sigma\left(L_{1}\right)\right)$ fit into a long exact sequence:

$$
\cdots \longrightarrow \widehat{H F}\left(\Sigma ( L _ { 0 } ) \longrightarrow \widehat { H F } \left(\Sigma\left(L_{1}\right) \longrightarrow \widehat{H F}(\Sigma(L) \longrightarrow \cdots,\right.\right.
$$

where the diagrams for $L_{0}$ and $L_{1}$ exhibit the two smooth resolutions of some crossing $c$ in $L$ and coincide with $L$ away from $c$. The existence of this sequence is a consequence of the surgery exact sequence for $\widehat{H F}$, and Ozsváth and Szabó use it to construct a spectral sequence whose $E^{2}$ term is isomorphic to the reduced Khovanov homology $\widetilde{K h}(\bar{L} ; \mathbb{Z} / 2 \mathbb{Z})$ of the mirror of $L$ and which converges to the Heegaard Floer homology group $\widehat{H F}(\Sigma(L) ; \mathbb{Z} / 2 \mathbb{Z})$. Let $\delta=(j-i)$ denote the quantum grading, the collapse of the bigrading on the group

$$
\widetilde{K h}(L)=\bigoplus_{i, j} \widetilde{K h}^{i, j}(L) .
$$

It was shown in $[\mathbf{1 0}]$ that the class of quasi-alternating links is Khovanovthin, with $\widetilde{K h}^{i, j}(L) \neq 0$ only if $\delta=(j-i)=-\sigma(L) / 2=\sigma(\bar{L}) / 2$. In the sequel [24], we describe a function $\underline{R}$ defined on a set of generators for $\widehat{C F}(\Sigma(K))$. If $K$ is $\rho$-degenerate, $\underline{R}$ indeed provides an absolute Maslov grading on $\widehat{H F}(\Sigma(K))$. In $[\mathbf{2 4}]$ we show that when $K$ is a two-bridge knot, then $K$ is $\rho$-degenerate and $\widehat{H F}(\Sigma(K))$ is supported entirely in the level $\underline{R}=\sigma(K) / 2$.

Baldwin $[\mathbf{2}]$ conjectured the existence of an induced $\delta$-grading on higher pages in the spectral sequence, and Greene [5] conjectured that a term arising in his spanning tree model could provide a quantum grading on $\widehat{H F}(\Sigma(K))$. If the gradings conjectured by Greene and Baldwin indeed exist, 
it would be interesting to compare them to the $\underline{R}$-grading for $\rho$-degenerate knots.

Furthermore, Szabó [23] constructed a geometric spectral sequence in $\mathbb{Z} / 2 \mathbb{Z}$ Khovanov homology. Although this spectral sequence is not known to abut to the Heegaard Floer homology, the construction is similar to that for the spectral sequence in [14]. Szabó's spectral sequence preserves the Khovanov $\delta$-grading, and it would be interesting to compare the induced grading on the $E^{\infty}$-page with the reduced function $\underline{R}$.

\section{References}

[1] M. Abouzaid and I. Smith, forthcoming sequel to The symplectic arc algebra is formal, 2013, arXiv: 1311.5535.

[2] J.A. Baldwin, On the spectral sequence from Khovanov homology to Heegaard Floer homology, Int. Math. Res. Not. IMRN 15 (2011), 3426-3470.

[3] S. Bigelow, A homological definition of the Jones polynomial, (2002), Invariants of knots and 3-manifolds (Kyoto, 2001), Geometry and Topology Monography 4, Geom. Topol. Publ., Coventry, 29-41 (electronic).

[4] J. Birman, On the stable equivalence of plat representations of knots and links, Can. J. Math. 28 (1976), 264-290.

[5] J. Greene, A spanning tree model for the Heegaard Floer homology of a branched double-cover, 2008, arXiv:math/0805.1381v1.

[6] A. Juhász and D. Thurston Naturality and mapping class groups in Heegaard Floer homology, 2012; arXiv:1210.4996v1.

[7] M. Kontsevich, Homological algebra of mirror symmetry, (1978), Proceedings of the International Congress of Mathematicians, Zürich, 1994, Birkhäuser, 120-139.

[8] R. Lipshitz, A cylindrical reformulation of Heegaard Floer homlogy Geom. Topol. 10 (2006), 955-1097.

[9] C. Manolescu, Nilpotent slices, Hilbert schemes, and the Jones polynomial, Duke Math. J. 132 (2006), 311-369.

[10] C. Manolescu and P. Ozsváth, On the Khovanov and knot Floer homologies of quasialternating links, Proceedings of the 14th gökova geometry-topology conference, 2007, 60-81.

[11] P. Ozsváth, and Z. Szabó, Absolutely graded Floer homologies and intersection forms for four-manifolds with boundary, Adv. Math. 173 (2003), 179-261.

[12] — Holomorphic disks and three-manifold invariants: properties and applications, Ann. Math. 159 (2004), 1159-1245.

[13] — Holomorphic disks and topological invariants for closed three-manifolds, Ann. Math. 159 (2004), 1027-1158.

[14] —, On the Heegaard Floer homology of branched double-covers, Adv. Math. 194 (2005), 1-33.

[15] — - Holomorphic triangles and invariants for smooth four-manifolds, Adv. Math. 202 (2006), 326-400.

[16] R. Rezazadegan, A spectral sequence for Lagrangian Floer homology, 2012; arXiv: $1201.4663 \mathrm{v} 3$. 
[17] J. Robbin and D. Salamon, The Maslov index for paths, Topology, 4 (1993), 827-844.

[18] S. Sarkar, Maslov index formulas for Whitney n-gons, J. Symplec. Geom. 9 (2011), 251-270.

[19] S. Sarkar and J. Wang, An algorithm for computing some Heegaard Floer homologies, Ann. Math. 171 (2010), 1213-1236.

[20] P. Seidel, Graded Lagrangian submanifolds, Bull. Soc. Math. France, 128 (2000), 103-146.

[21] P. Seidel and I. Smith, A link invariant from the symplectic geometry of nilpotent slices, Duke Math. J. 134 (2006), 453-514.

[22] P. Seidel and I. Smith, Localization for involutions in Floer cohomology, Geom. Funct. Anal., 20(6) (2010), 1464-1501.

[23] Z. Szabó, A geometric spectral sequence in Khovanov homology, 2010; arXiv: $1010.4252 \mathrm{v} 1$.

[24] E. Tweedy, The anti-diagonal filtration: reduced theory and applications, 2011; arXiv: $1109.3425 \mathrm{v} 1$.

[25] J. Waldron, An invariant of link cobordisms from symplectic Khovanov homology, 2009; arXiv:0912.5067.

\section{Department of Mathematics}

Rice University

Houston, TX 77005

E-mail address: eamonn@rice.edu

Received 09/20/2011, accepted 06/25/2013

It is my pleasure to thank Ciprian Manolescu for suggesting this problem to me and for his invaluable guidance as an advisor. I would also like to thank Robert Lipshitz, Liam Watson, and Tye Lidman for some instructive discussions, Stephen Bigelow for some helpful email correspondence related to his paper [3], Yi Ni for some useful comments regarding relative Maslov gradings. This paper has been rewritten from a previous version to account for an update to the paper [22]. I am indebted to Ivan Smith for pointing out this change.

I would also like to thank the anonymous Referee, whose corrections and suggestions led to countless improvements in this article. 
